\title{
Synaptic Vesicles Studied by Small-Angle X-Ray Scattering
}

\author{
Dissertation \\ zur Erlangung des Doktorgrades \\ der Mathematisch-Naturwissenschaftlichen Fakultäten \\ der Georg-August-Universität zu Göttingen
}

vorgelegt von

Simon Johannes Castorph

aus Göttingen

Göttingen 2010 
D7

Referent: Prof. Dr. Tim Salditt

Korreferent: Prof. Dr. Marcus Müller

Tag der mündlichen Prüfung: 14. Juni 2010 


\section{Contents}

1 Introduction $\quad 1$

2 Synaptic Vesicles $\quad 5$

2.1 Trafficking Organelle . . . . . . . . . . . . . . . . . . . 5

2.2 Molecular Inventory . . . . . . . . . . . . . . . . . . . . . 6

2.3 Purification . . . . . . . . . . . . . . . . . . 6

2.4 Purity Assessment: Shape and Size Polydispersity Characterization . . . . . 8

2.5 Cryogenic Electron Microscopy _. . . . . . . . . . . . . . . 8

2.6 Dynamic Light Scattering . . . . . . . . . . . . . . . . . . 10

2.6.1 Autocorrelation Function . . . . . . . . . . . . . . . 11

2.6.2 Data Regularization and Inversion . . . . . . . . . . . . . . 11

2.6.3 Direct Modeling . . . . . . . . . . . . . . . . . . . . . . . 12

2.6.4 Asymmetric-Flow Field-Flow Fractionation . . . . . . . . . . . . . . 14

2.6.5 Instrumentation and Choice of Parameters . . . . . . . . . . . . . 14

2.6.6 Results . . . . . . . . . . . . . . . . . . . 15

2.6.7 Discussion and Conclusions . . . . . . . . . . . . . . . . . . 19

2.7 Sample Preparation for X-Ray Scattering Experiments . . . . . . . . . . . . 21

2.7.1 Native Synaptic Vesicles . . . . . . . . . . . . . . . . . . . . . . 21

2.7.2 Protease Treatment Synaptic Vesicles . . . . . . . . . . . . . . . . 21

2.7.3 Preparation of Liposomes . . . . . . . . . . . . . . . . . . . . 21

2.8 Conclusions . . . . . . . . . . . . . . . . . . . . 22

3 Small-Angle X-Ray Scattering $\quad 23$

3.1 Experiments and Instrumental Corrections . . . . . . . . . . . . . . . . . . . 23

3.1 .1 Beamline ID-2, ESRF . . . . . . . . . . . . . . . 24

3.1.2 Beamline B1, HASYLAB . . . . . . . . . . . . . . . 28

3.2 Results . . . . . . . . . . . . . . . . . . . . . . 29

3.3 Conclusions . . . . . . . . . . . . . . . . . . . 32 
4 Data Modeling of X-Ray Scattering Curves $\quad 35$

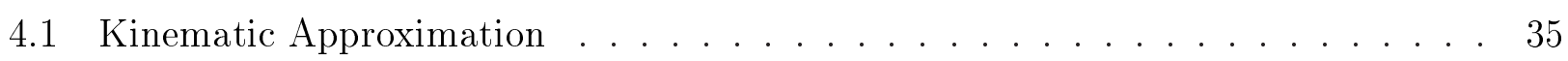

4.2 Model Independent Data Analysis . . . . . . . . . . . . . . . . . . 36

4.2.1 Indirect Fourier Transformation Method and Convolution Square Root Operation ............................. 36

$4.2 .2 \quad$ Ab-Initio Shape Analysis . . . . . . . . . . . . . . . . . . . . . 37

4.3 Direct Modeling . . . . . . . . . . . . . . . . . . . . . . 37

4.4 Model Building: Integrated Approach . . . . . . . . . . . . . . . . 37

4.5 Derivation of Form Factors . . . . . . . . . . . . . . . . . . . 40

4.5.1 Isotropic Models . . . . . . . . . . . . . . . . . 40

4.5.2 Anisotropic Models . . . . . . . . . . . . . . . . . . . 41

4.6 Model Falsification Against SAXS Data . . . . . . . . . . . . . . . 49

4.6 .1 Native Synaptic Vesicles . . . . . . . . . . . . . . . . . . 51

4.6 .2 Model Liposomes . . . . . . . . . . . . . . . . . . . . . . . . 56

4.6.3 Protease Treated Synaptic Vesicles . . . . . . . . . . . . . . . . . . 59

4.7 Conclusions . . . . . . . . . . . . . . . . . . . . . . 60

5 Model Evaluation and Structural Results $\quad 63$

5.1 Model Interpretation and Discussion . . . . . . . . . . . . . . 63

5.1 .1 Size Polydispersity . . . . . . . . . . . . . . . 63

5.1 .2 Bilayer Structure . . . . . . . . . . . . . . . . 65

5.1.3 Model Dependencies and Ambiguities . . . . . . . . . . . . . . . . 66

5.2 Model Entropy Considerations . . . . . . . . . . . . . . . . . . . . 68

5.2 .1 Cluster Formation and Disintegration . . . . . . . . . . . 68

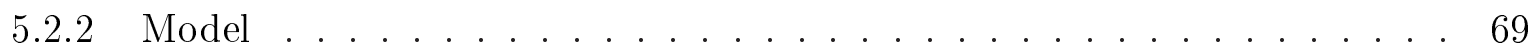

5.2 .3 Results............................ 70

5.3 Conclusions . . . . . . . . . . . . . . . . . 70

6 Towards Cell Free Fusion Systems $\quad 73$

6.1 Introductory Considerations . . . . . . . . . . . . . . . . . . 73

6.2 Samples . . . . . . . . . . . . . . . . . . . . . . 77

6.3 Dynamic Light Scattering . . . . . . . . . . . . . . . . . 77

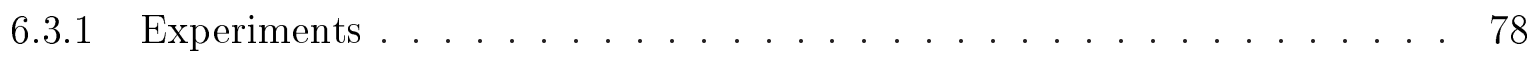

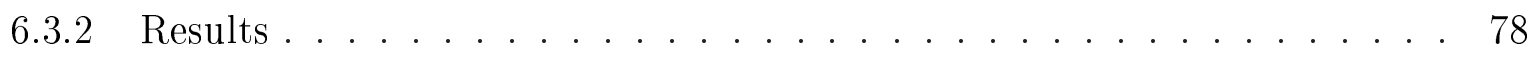



6.4 Small-Angle X-Ray Scattering . . . . . . . . . . . . . . . . . 80

6.4.1 Gedankenexperiment . . . . . . . . . . . . . 80

6.4.2 Resulting Calculated Scattering Curves . . . . . . . . . . . . . 82 


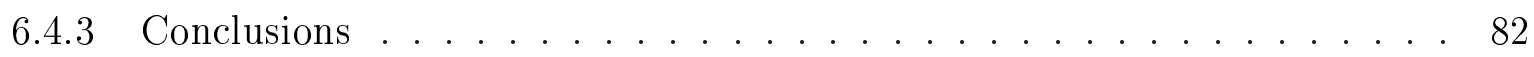



$\begin{array}{lll}7 & \text { Summary } & 85\end{array}$

$\begin{array}{lr}\text { Bibliography } & 89\end{array}$

$\begin{array}{ll}\text { Appendix A MATLAB Source Code } & 99\end{array}$

A.1 Direct Modeling Dynamic Light Scattering Data . . . . . . . . . . . . . . . . 99

A.1.1 ObjFunVec_DLS.m . . . . . . . . . . . . . . . . 99

A.2 Direct Modeling Small-Angle X-Ray Scattering Data . . . . . . . . . . . . 102

A.2.1 ObjFunVec_SAXS.m . . . . . . . . . . . . . 102

A.3 Model Entropy Calculations . . . . . . . . . . . . . . . . . 107



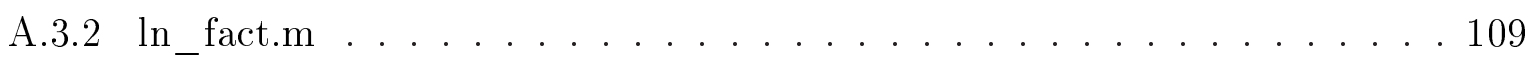

$\begin{array}{ll}\text { Appendix B List of Publications } & 111\end{array}$

$\begin{array}{ll}\text { Appendix C Acknowledgements } & 113\end{array}$ 


\section{Chapter 1}

\section{Introduction}

The complex higher functions of the central and peripheral nervous systems are intimately related to the signaling of neurons and the information flow in the spatially organized, complex neuronal circuitries. The entire human neuronal network consists of about $10^{10}$ to $10^{12}$ neurons $^{1}(2)$. Typically, each of these neurons forms about $10^{3}$ chemical synapses $^{2}$ (3), connecting the neuron to other cells, or back to itself. The presynaptic terminus at each chemical synapse typically contains about $10^{3}$ synaptic vesicles (4), small membranous organelles, typically encapsulating in the order of $10^{3}$ to $10^{4}$ neurotransmitter molecules in their interior (4).

On a sub-cellular and molecular level, the understanding of the processes related to neuronal signaling is intimately related to the elucidation of the processes of membrane merger and budding in intracellular membrane trafficking in neurons $(5,6)$, and in particular to the fusion of synaptic vesicles with the plasma membrane leading to exocytosis of neurotransmitter molecules at chemical synapses $(7)$.

The basic physical principles of molecular interactions, surface forces and curvatures governing membrane merger and budding (8) are directly related to the dynamical self-assembly of macromolecules, including lipid molecules and a multitude of different proteins, into highly complex structures, such as membranes, vesicles, micelles, microemulsions or complex aggregates, both in vivo and ex vivo (9).

Figure 1.1 illustrates a chemical synapse, a unidirectional communication channel allowing the presynaptic cell to signal the postsynaptic cell. Upon arrival of an action potential through the neuronal axon of the presynaptic cell, synaptic vesicles fuse $\left[\mathrm{Ca}^{2+}\right]$-dependent with the plasma-membrane, releasing their neurotransmitter content into the synaptic cleft. The neurotransmitter molecules diffuse to the postsynaptic cell and are recognized on the surface of the postsynaptic cell by receptors, leading either to excitation via the generation of an action poten-

\footnotetext{
${ }^{1}$ The number of stars in the Milky Way galaxy is estimated to be of about the same order of magnitude (1).

${ }^{2}$ In humans, chemical synapses outnumber electrical synapses by far (2). Thus we neglect the number of electrical synapses here.
} 


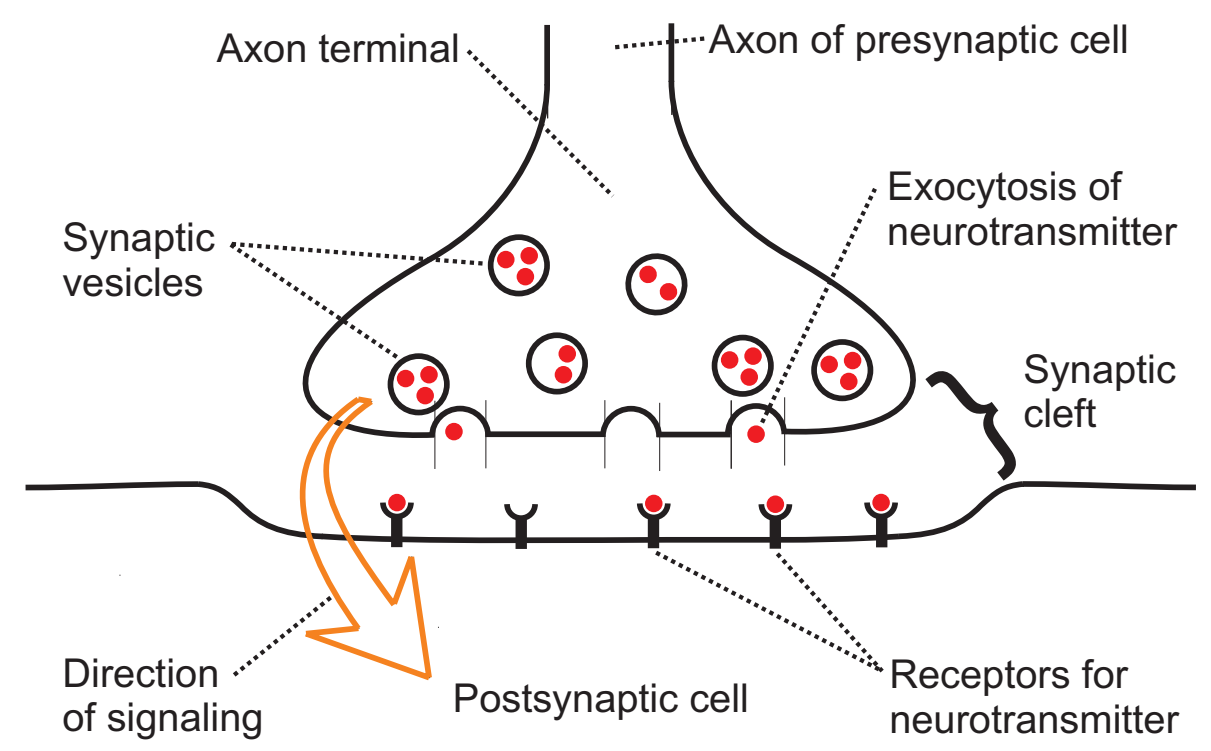

Figure 1.1: The synaptic cleft separates the plasma membranes of the presynaptic and postsynaptic cells. Continued transmission of electric nervous impulses is mediated via the release of a neurotransmitter (red circles) by the presynaptic cell, its diffusion across the synaptic cleft, and its binding to specific receptors on the plasma membrane of the postsynaptic cell. Simplified model sketch, adapted from (3).

tial in the postsynaptic cell, or to the inhibition of the postsynaptic cell by hyperpolarization. Synaptic vesicle components are recovered from the plasma membrane by clathrin-dependent neuronal endocytosis. The synaptic vesicle eventually refills with neurotransmitter and can participate in a new round of neuronal exocytosis.

Figure 1.2 illustrates some important molecular processes in the fusion of the synaptic vesicle with the plasma membrane of the presynaptic cell $(11,12)$. Neuronal exocytosis is mediated and controlled by the $\mathrm{SNARE}^{3}$ proteins synaptobrevin 2 (black), anchored in the synaptic vesicle membrane, syntaxin 1 (cyan) and SNAP-25 (green), both anchored in the plasma membrane of the presynaptic cell. SNARE proteins comprise a superfamily of small membrane-bound proteins, sharing a common SNARE-motif. The current model of synaptic vesicle fusion (13) implies that the SNARE-motifs of synaptobrevin 2, SNAP-25 $5^{4}$ and syntaxin 1 assemble into elongated four-helix trans-complexes, connecting the membrane of the synaptic vesicle with the plasma membrane. The energy barrier separating the membranes may be overcome by the energy provided by the progressing assembly process, initializing fusion. After fusion of the membranes, the formed complexes are aligned parallelly in the plasma membrane of the

\footnotetext{
${ }^{3}$ Acronym derived from Soluble N-ethylmaleimide-sensitive factor Attachment protein REceptors.

${ }^{4}$ Synaptosomal-associated protein
} 


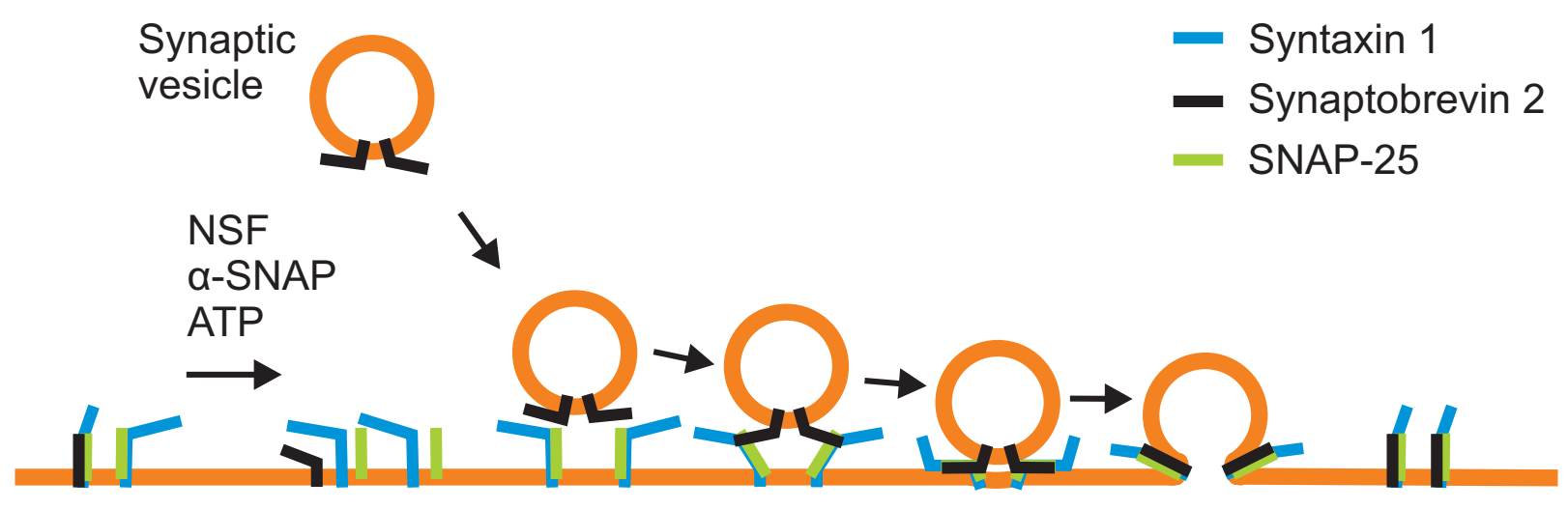

SNARE regeneration trans-complex formation cis-complex formation

Figure 1.2: On a molecular scale, SNARE proteins mediate and control the fusion process of synaptic vesicles with the plasma membrane of the presynaptic cell upon neuronal exocytosis, as detailed in the text. Simplified model sketch, after (10).

presynaptic cell (cis complexes). $\mathrm{NSF}^{5}$ and $\mathrm{SNAPs}^{6}$ disassemble the cis complexes under $\mathrm{ATP}^{7}$ consumption, reversing the assembly processes and regenerating the SNARE proteins for an other round of fusion.

The synaptic vesicle as a key player in neuronal exocytosis has been investigated and physically characterized by employing a multitude of analytical techniques including cryogenic electron microscopy, scanning transmission electron microscopy, and fluorescence correlation spectroscopy. The stoichiometry of individual constituent protein and lipid molecules has been addressed by biochemical analysis. Further, x-ray crystallography elucidated structures of several proteins found on the synaptic vesicle. Recently, the enormous progress in this field culminated in a molecular model of the entire average synaptic vesicle isolated from rat brain (14).

However, we still lack detailed empirical data on the structure of the entire synaptic vesicle elucidating details on the density profile of the membrane, including contributions from lipids and proteins, as well as addressing the average conformation and overall organization of proteins on synaptic vesicles under quasi-physiological conditions. Such structural information may contribute to describing and understanding the processes of membrane fusion, retrieval and recycling related to neuronal exocytosis, and to membrane trafficking in eukaryotic cells in general.

Based on these considerations and works, the aim of this thesis is

(i) to contribute to the understanding of the synaptic vesicle (SV) structure, and to the understanding of the processes of neuronal exocytosis and endocytosis, prominent examples of

\footnotetext{
${ }^{5}$ N-ethylmaleimide-sensitive factor

${ }^{6}$ Soluble NSF Attachment Proteins

${ }^{7}$ Adenosine triphosphate
} 
membrane trafficking in cells, (ii) to prepare and to characterize samples of SVs isolated from rat brain suited for investigation by small-angle x-ray scattering (SAXS), and to record solution SAXS data from SV dispersions under quasi-physiological conditions, (iii) to develop structural models for SVs, to calculate the corresponding scattering form factors, and to optimize and to falsify these form factors against experimentally determined scattering curves from SVs, (iv) to contribute to the understanding of fusion pathways by developing new approaches for structural investigation of cell free fusion systems.

Sections of the thesis are partly based on manuscipts which will be or have been published elsewhere, as indicated in detail below. The thesis is organized as follows:

Chapter 2 introduces the SV as model trafficking organelle, presents the molecular inventory of SVs, and describes a purification protocol for the isolation of SVs from rat brain (15). The purity of the SV dispersions obtained from the isolation protocol is characterized by cryogenic electron microscopy (cryo-EM) (15), and dynamic light scattering (DLS) (16), and are further purified by asymmetric-flow field-flow (AFFF) fractionation (16). Further, the preparation of samples for x-ray scattering experiments is addressed $(15,17)$.

Chapter 3 presents results of synchrotron-based small-angle x-ray scattering measurements under quasi-physiological conditions from dispersed SVs isolated from rat brain (15, 17). Aggregation and strong interaction potentials between SVs are excluded.

Chapter 4 discusses different model independent and model dependent approaches to model SAXS curves. Different isotropic and anisotropic SV models are developed and corresponding scattering form factors are calculated $(15,17)$. Subsequently, the form factors are least-squares fitted to SAXS curves $(15,17)$. All investigated isotropic form factors are falsified $(15,17)$. Two anisotropic form factors are presented which are in excellent agreement with SAXS data, cryo-EM observations, biochemical data and DLS data $(15,17)$. The SV size polydispersity distribution, and the electron density profile of the protein decorated SV bilayer is given on an absolute scale with no free prefactors (15).

Chapter 5 presents an evaluation and discussion of the optimized form factor models $(15,17)$. The optimized parameter values of the model form factors are indicative of larger protein clusters on the SV membrane $(15,17)$. Possible model dependencies and ambiguities are addressed $(15,17)$. Entropic contributions to the free energy due to protein cluster formation and disintegration on the SV is investigated by calculating the entropy of a microcanonical SV model.

Chapter 6 presents work on a cell free fusion system, employing SVs and proteo-liposomes with reconstituted SNARE proteins (16). The capabilities of using DLS to quantify fusion processes is assessed (16). A SAXS Gedankenexperiment is considered, and calculated scattering curves of fused SVs and proteo-liposomes are presented and discussed (18).

Chapter 7 finally summarizes the results and presents the conclusions $(15,16,17,18)$. 


\section{Chapter 2}

\section{Synaptic Vesicles}

This chapter introduces the function and molecular inventory of SVs, and describes the methods to isolate and assess the purity of SVs from rat brain. These techniques allow to prepare samples of dispersed SVs under quasi-physiological conditions, suited for investigation by solution SAXS experiments. The SV is characterized as a trafficking organelle, the key player in neuronal exocytosis, a temporally and spatially highly controlled process (Section 2.1). Details of the biochemical composition of SVs, including lipid and protein inventory, have been elucidated in recent years (Section 2.2). A purification protocol (Section 2.3) of SVs from rat brain has opened up numerous possibilities to investigate native SVs ex vivo. The purity (Section 2.4) of the obtained SV dispersions is investigated and characterized by cryo-EM (Section 2.5) and DLS (Section 2.6). Finally, the preparation of samples for solution SAXS experiments is described (Section 2.7).

\subsection{Trafficking Organelle}

Synaptic vesicles (SVs) are secretory trafficking organelles that store neurotransmitter in presynaptic nerve endings (14). When an action potential arrives in the nerve terminal, the plasma membrane is depolarized leading to the opening of voltage-gated $\left[\mathrm{Ca}^{2+}\right]$ channels in the plasma membrane. The accompanying rise in intracellular $\left[\mathrm{Ca}^{2+}\right]$ leads to the fusion (exocytosis) of the synaptic vesicles with the plasma membrane, resulting in the release of neurotransmitter. Following exocytosis, SV membrane is recovered by endocytosis and used to reform vesicles which are then refilled with neurotransmitter molecules and used for a subsequent round of exocytosis (19). As the SV is the only constant during this cycle, it must be able to co-ordinate the process (15). 


\subsection{Molecular Inventory}

In a primary approach to understanding SV function, individual proteins on isolated vesicles were identified and their functions elucidated, such as synaptobrevin which is the SNARE protein thought to play a role in exocytosis (20). A preliminary analysis of lipid composition was also performed (21). Work from several laboratories over the years culminated in the recent publication of a molecular model that attempted to integrate all quantitative data on the protein and lipid composition of the vesicle (14), see Fig. 2.1. The protein contributions included in the model account for approximately $67.5 \%$ of the estimated total mass of all proteins on the vesicle (14). Despite these efforts, what is still lacking is an empirical description of SV structure at the supra-molecular level, which is necessary to fully describe and understand the processes of membrane fusion, retrieval and recycling. Importantly, such an assessment of SV structure, compatible with more physiological conditions and with higher (near molecular) resolution, can be effectively cross-validated by these recent, independent studies. Unfortunately, the property that allows vesicle purification (small size) complicates structural analysis. For instance, advanced light microscopy techniques, e.g. photo activated localization microscopy (PALM) (22) or stimulated emission depletion microscopy (STED) (23), or advanced nanoscale x-ray imaging techniques (24), are at the limit of the spatial resolution required. In contrast, electron microscopy techniques, such as cryo-EM and quick-freeze deep etch microscopy, can provide detailed structural information on the conformation of protein (complexes), but both fail to provide detailed structural information about the lipid environment of the protein (complex) under investigation. Further, care has to be taken as these methods are prone to method-specific artifacts. SAXS, on the other hand, is a well-established technique that has traditionally been used for the ensemble solution structure of biomolecules (25) or larger, regular shaped structures such as virus capsids (26). Importantly, the technique is also capable of providing detailed information about lipid structures and associated proteins, under quasiphysiological conditions. Here we demonstrate that SAXS is an ideal technique to study the (heterogeneous) supramolecular structure of a functional organelle on an absolute scale.

\subsection{Purification}

Fortunately, the analysis of SVs is simplified by the fact that they can be purified to apparent homogeneity in large quantities, making them amenable to biochemical studies. This purification is possible because they are very abundant in brain tissue (approximately $5 \%$ of the protein in the central nervous system) and smaller and more homogeneous in size and shape than most other organelles, allowing the application of mild size fractionation techniques. 

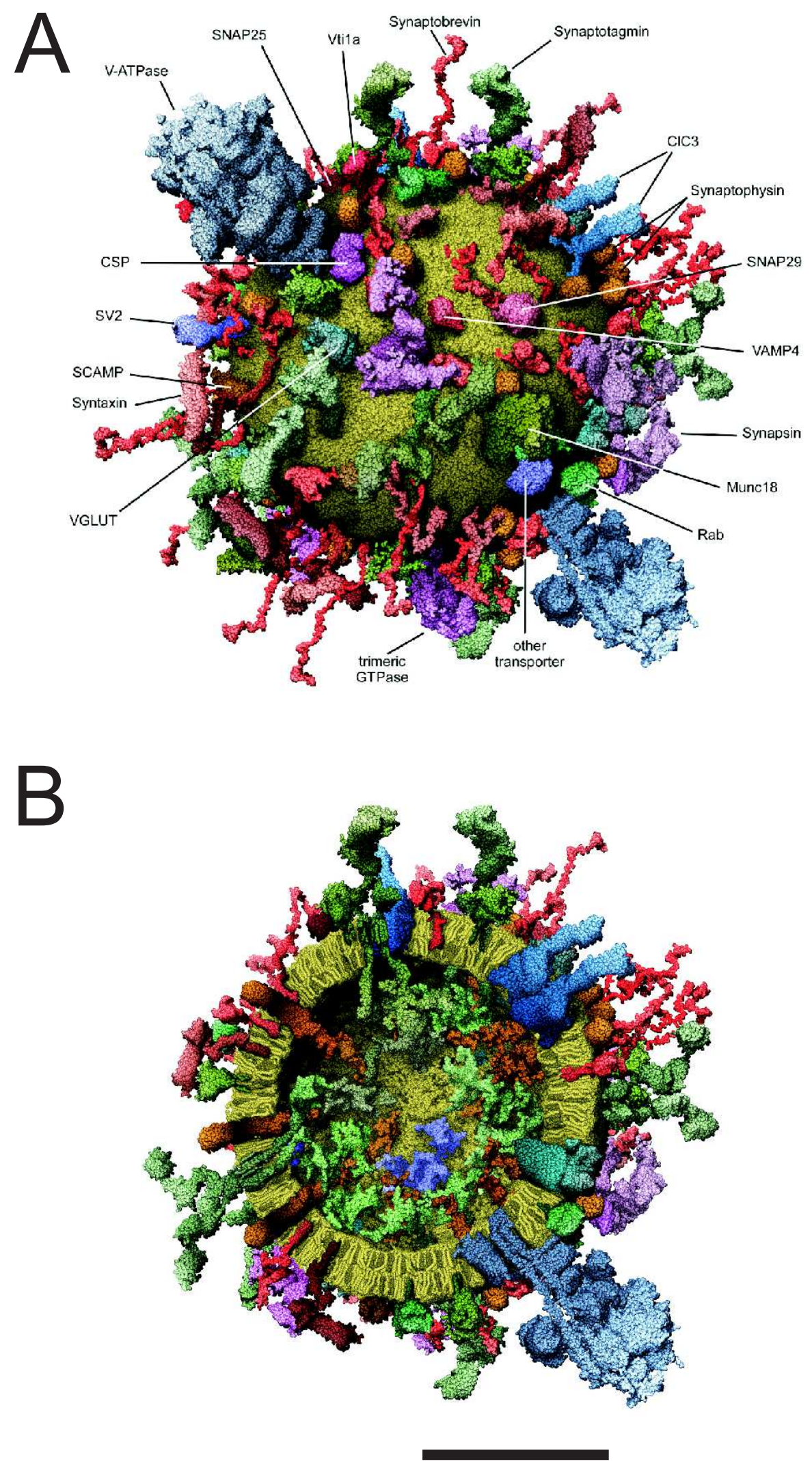

Figure 2.1: (A) Outside view, and (B) section through a molecular model of an average SV isolated from rat brain, based on space-filling models of macromolecules at near-atomic resolution. Scalebar $20 \mathrm{~nm}$. Reproduction from (14). 
Synaptic vesicles were purified by Matthew Holt ${ }^{1}$ from rat brain, as described (14), through differential centrifugation, sucrose density centrifugation and size exclusion chromatography. While SVs prepared this way are $95 \%$ pure (as measured by immunogold electron microscopy for integral SV membrane proteins), some larger membranous structures remain following purification $(100-200 \mathrm{~nm})$. These particles (less than $0.9 \%$ of the total number of particles) have a significant influence on the scattering intensity. Analytical tools were developed to account for this (see later). Following chromatography, an additional centrifugation step was introduced to allow buffer exchange and SV concentration. SVs were resuspended in HB100 (in mM; $100 \mathrm{KCl}$, $1 \mathrm{DTT}^{2}, 25 \mathrm{HEPES}^{3}$, pH $\left.7.40 \mathrm{KOH}\right)$, and immediately snap-frozen for transportation to the synchrotron. Importantly, membrane damage due to freeze/thaw was minimal as judged by the capacity of the SVs to acidify (27). The dry weight of the SV population was obtained by measuring the protein mass using a modified Lowry assay and assuming a constant (10:5:2) ratio of proteins, phospholipids and cholesterol (14). The resulting SV stock solutions had a protein concentration of about $6 \mu \mathrm{g} / \mu l$.

The purity of the SV samples was subsequently checked and quantified by cryo-EM and DLS.

\subsection{Purity Assessment: Shape and Size Polydispersity Char- acterization}

To assess the purity of the SV dispersions, the shape of the SVs and the relative size polydispersity $p_{n}(R)$ of the SV population were measured by cryo-EM on vitrified SV solutions. To characterize the larger membranous particles, EM tilt-pair images were taken.

Further, to assess $p_{n}(R)$ under quasi-physiological conditions, and to quantify the contributions from larger trace particles present in the SV samples, DLS measurements were performed at diluted SV dispersions. Asymmetric-flow field-flow (AFFF) Fractionation was used for further purification of the SV dispersions, effectively eliminating the contaminant larger trace particles.

\subsection{Cryogenic Electron Microscopy}

Cryo-EM measurements were performed by Dietmar Riedel ${ }^{4}$ on vitrified SV solutions using a Philips Titan Krios (Cs corrected) microscope operating at $300 \mathrm{kV}$, and equipped with a FEI

\footnotetext{
${ }^{1}$ Department of Neurobiology, Max Planck Institut für Biophysikalische Chemie, Göttingen, Germany

${ }^{2}$ Dithiothreitol, (2S,3S)-1,4-Bis-sulfanylbutane-2,3-diol.

${ }^{3} 2$-[4-(2-hydroxyethyl)piperazin-1-yl]ethanesulfonic acid

${ }^{4}$ Department of Neurobiology, Max Planck Institut für Biophysikalische Chemie, Göttingen, Germany
} 



Figure 2.2: (A) EM size distribution of SVs from rat brain. ( $B$ and $C$ ) Cryo-EM images of typical SVs from rat brain. Scale bars $20 \mathrm{~nm}$. ( $D$ and E) Cryo-EM tilt-pair images of SVs and larger membranous trace particles present in the SV dispersions. $0^{\circ}$ and $45^{\circ}$ relative angles, scale bars $50 \mathrm{~nm}$. Figure partly adapted from (15).

Eagle 4k CCD, running in 2-fold binning mode (FEI, Holland). Samples were vitrified in a fully automated Vitribot Mark IV (FEI) vitrification device for plunge-freezing of aqueous suspensions providing a tightly controlled sample environment (temperature, humidity), allowing to avoid cooling and concentration artifacts, often unavoidable in other freezing methods. Samples were first bound to a glow discharged holey carbon foil (quantifoil grid), and blotted twice with filter paper for one second at blot-force 2 . Samples are kept at $30^{\circ} \mathrm{C}$ and $97 \%$ relative humidity, and subsequently vitrified and transferred from the vitrification medium into the liquid nitrogen atmosphere. In total, $559 \mathrm{SVs}$ were measured and $R$ was determined by taking the average of the shortest and longest diameter of the SVs, as measured from bilayer surface to bilayer surface.

To characterize the larger membranous particles, EM tilt-pair images at $0^{\circ}$ and $45^{\circ}$ relative angles were taken with a Philips CM200 FEG microscope and recorded using a TVIPS 4k x 4k slow scan CCD, running in 2-fold binning mode (FEI). These were used to assess the sampling error caused by uneven collapse of particles onto the carbon grid.

Figure 2.2 (A) shows the size distribution $p_{n}(R)$ of $\mathrm{SVs}$ as determined by the analysis of cryo-EM images of $559 \mathrm{SVs}$. The SV radius $R$ was determined from the diameter of the $\mathrm{SV}$, measured from bilayer surface to bilayer surface. The most frequently occurring size is $R=21 \mathrm{~nm}$. The size distribution runs from $R=15$ to $R=30 \mathrm{~nm}$, and drops off asymmetrically 
with a slower descent towards larger radii than towards smaller ones. ( $B$ and $C$ ) show typical cryo-EM micrographs of SVs. Clearly visible are proteins extending both to the outside and the lumen of the SV, and the characteristic lipid bilayer structure. No aggregation of SVs was observable by electron microscopy. ( $D$ and $E$ ) show typical cryo electron micrographs (tilt pairs) of SVs and larger membranous trace particles present in the SV dispersions, which seemed to be unavoidable in the purification protocol, e.g. due to column bleed. These particles may be formed by larger membrane aggregates, possibly originating from early endosomes or unspecific vesiculated membranes. For further details see (14).

Due to the small number of these larger particles when compared to the number of SVs, it is practically impossible to precisely quantify the relative contributions from SVs and the larger trace particles by cryo-EM. However, such an assessment is possible by using scattering techniques like DLS or SAXS as the contributions to the scattering signal depend highly non linearly on the particle size.

\subsection{Dynamic Light Scattering}

Dynamic Light Scattering (DLS) can be employed for detailed characterizations of size polydispersities of SVs under quasi-physiological conditions, see diploma thesis of Sarah Schwarz Henriques $^{5}$ (28). Measurements are fast and economic when compared to cryo-EM, promising to open up the possibility of following the dynamics of polydispersity distributions in real-time.

We find that DLS autocorrelation curves can consistently be described by a model calculation employing a size distribution function $p_{n}(R)$ obtained by cryo-EM of vitrified SV dispersions (16).

The size distribution of the native SV preparation contains a second trace population of larger particles $(14,15)$. Asymmetric-flow field-flow (AFFF) fractionation is used for further purification of the SV samples, effectively eliminating the larger contaminant particles (16). DLS spectra of SV populations can be regularized and inverted, giving direct access to the size distribution of the SV which is in excellent agreement with cryo-EM and SAXS data. While the inversion approach works well in the case of SV samples purified by means of AFFF, it fails to resolve the bimodal size distribution if larger trace particles are present in the sample. Instead, a mono-modal distribution is obtained, shifted slightly towards larger radii and showing a somewhat larger width (16).

\footnotetext{
${ }^{5}$ Institut für Röntgenphysik, Georg-August-Universität Göttingen
} 


\subsubsection{Autocorrelation Function}

For a dilute dispersion of polydisperse spherical particles of hydrodynamic radii $R_{h}$ in Brownian motion, $g_{1}(\tau)$ is the Laplace transform with respect to the hydrodynamic radii $R_{h}$ given by $(29)$

$$
g_{1}(\tau)=\int d R_{h} p_{n}\left(R_{h}\right) V\left(R_{h}\right)^{2}\left|F\left(q, R_{h}\right)\right|^{2} \exp \left(\frac{-k_{B} T}{6 \pi \eta R_{h}} q^{2} \tau\right),
$$

with $\tau$ the delay time, $p_{n}\left(R_{h}\right)$ the distribution of particle sizes, $V\left(R_{h}\right)$ the volume of the particles, or the particle shells, $F\left(q, R_{h}\right)$ the normalized form factor amplitudes of the particles, $k_{B}$ Boltzmann's constant, $T$ temperature, and $\eta$ the viscosity of the buffer. The form factor amplitude $F_{s}\left(q, R_{h}\right)$ for a homogeneous sphere of hydrodynamic radius $R_{h}$, calculated within the Rayleigh-Debye-Gans theory, is given by (30)

$$
F_{s}\left(q, R_{h}\right)=3 \frac{\sin \left(q R_{h}\right)-q R_{h} \cos \left(q R_{h}\right)}{\left(q R_{h}\right)^{3}},
$$

with $q=\frac{4 \pi}{\lambda} \sin (\Theta)$ the modulus of the scattering vector, $\Theta$ the scattering angle, and $\lambda$ the wavelength of the incident photons and the elastically scattered photons. For hollow spheres of hydrodynamic radius $R_{h}$ and shell thickness $t$ the form factor amplitude $F_{h s}\left(q, R_{h}\right)$ is given correspondingly by (30)

$$
F_{h s}\left(q, R_{h}\right)=F_{s}\left(q, R_{h}\right)-F_{s}\left(q, R_{h}-t\right) .
$$

Considering that the inverse Laplace transformation of $g_{1}(\tau)$ with respect to time is a wellknown 'ill-conditioned' problem, we choose different approaches to tackle this problem and to analyze $g_{1}(\tau)(31)$.

\subsubsection{Data Regularization and Inversion}

A nonlinear fit to $\left|\beta g_{1}(\tau)\right|^{2}$ is calculated by using a constrained regularization method (32, 33, 34 ), employing a CONTIN algorithm $(35,36)$ in a standard implementation (ALV-Correlator Software ALV-7004 for Windows, V.3.0.4.5) by ALV GmbH, Langen, Germany. For the data analysis, several settings are specified within the $A L V$-Regularized fit setup of the ALVCorrelator Software. If not indicated differently, the fit model DLS-Exponential $\left(g_{2}(t)\right)$ is used, and fit additional baseline as well as enable data weighting is enabled. Further, single fit with target $P R O B 1=0.5$ is selected. The fit range is chosen to be between $250 \mathrm{~ns}$ and $78.6 \mathrm{~ms}$. To estimate the errors of the correlation function at each lag time, the ALV software uses a theoretical model described in $(31,37,38)$. The particles are modeled as hard spherical shells of thickness $12 \mathrm{~nm}$, as suggested by the low resolution structure proposed in (15), or as hard spheres (see section 6.3). Correspondingly, fit for vesicles with $r^{*}=12 \mathrm{~nm}$ was enabled, or disabled within the ALV-Regularized fit setup of the ALV-Correlator Software. The result of the 
inversion $p_{I}\left(R_{h}\right)=p_{n}\left(R_{h}\right) V\left(R_{h}\right)^{2}\left|F\left(q, R_{h}\right)\right|^{2}$ is the intensity weighted distribution function, a measure of the contribution from the differently sized vesicles to the auto-correlation function. To obtain a size distribution function $p_{n}\left(R_{h}\right), p_{I}\left(R_{h}\right)$ needs to be corrected for the particle form factor $F\left(q, R_{h}\right)$ and volume $V\left(R_{h}\right)$. The calculation of $F\left(q, R_{h}\right)$ is performed by modeling the synaptic vesicles as hard spherical shell particles with a shell thickness of $t=12 \mathrm{~nm}$, or as hard spheres (see Eq. 2.3 and Eq. 2.2, above). As the roots in $F\left(q, R_{h}\right)$ would lead to singularities in $p_{n}\left(R_{h}\right)$, the particle form factor is smoothed around these points. Both $p_{I}\left(R_{h}\right)$ and $p_{n}\left(R_{h}\right)$ are calculated by the ALV software. Normalization sets the highest peak to 1.

Figure 2.3 (A) shows DLS data from a native SV ensemble. A polydisperse size distribution (Fig. 2.3 (B) was obtained from the data set by a regularized nonlinear inversion of the intensity correlation function $g_{2}(\tau)-1$. The inversion was performed by the ALV software, yielding first the intensity weighted distribution $p_{I}\left(R_{h}\right)$ (black circles). Experimental errors were estimated by the ALV software according to the theoretical model described in $(31,37,38)$. Correcting for the particle volume and form factor the size distribution $p_{n}\left(R_{h}\right)$ (full black squares) was then calculated from $p_{I}\left(R_{h}\right)$.

The width and shape of the distribution $p_{n}\left(R_{h}\right)$ are to some degree influenced by the regularization. The maximum at $20.9 \mathrm{~nm}$ is, however, found to be independent of the regularization. A second peak at $161 \mathrm{~nm}$ arises from the correction for the particle form factor. Around that radius the form factor takes values close to zero, making it difficult to determine the relative number of particles within that particular size range.

\subsubsection{Direct Modeling}

In a direct approach $g_{2}(\tau)$ is analyzed by least-square fitting to a model of polydisperse hard spherical shell particles, undergoing independent (uncorrelated) Brownian motion, using the lsqnonlin routine of MATLAB Optimization Toolbox (Version 7.5.0.342 (R2007b), The MathWorks Inc.), dedicated to solve nonlinear least-squares problems. A bimodal size distribution was assumed consisting of a fixed part and a freely varied Gaussian distributed component. The constant part describes the size distribution of SV as determined by cryo-EM, shifted by $3 \mathrm{~nm}$ towards larger radii to account for proteins on the outer surface of the SV bilayer. The Gaussian distribution accounts for larger membranous trace particles. A constant shell thickness of $12 \mathrm{~nm}$ is assumed for the particles, in agreement with structural parameters of the protein decorated SV bilayer as determined by SAXS (15). Further details on the MATLAB code are given in the appendix. 



Figure 2.3: (A) DLS data of a native SV ensemble. (B) Intensity weighted distribution $p_{I}\left(R_{h}\right)$ (black circles) and size distribution $p_{n}\left(R_{h}\right)$ (full black squares) obtained by a regularized inversion of the data shown in $(A)$. 


\subsubsection{Asymmetric-Flow Field-Flow Fractionation}

Asymmetric-flow field-flow (AFFF) fractionation is a one-phase chromatography technique which allows to separate particles of different sizes on the basis of their diffusion properties(39, 40, 41). The dispersed particles travel along a channel with a parabolic velocity profile in the principal flow direction. An additional small perpendicular force field is applied which drives the particles towards one wall of the flow channel. The particles exhibit an equilibrium position in the direction of the perpendicular force field which depends on their diffusion associated with Brownian motion. Thus, the particles exhibit different travel velocities in the direction of the principal flow. Smaller particles will reach the end of the channel faster than larger particles, and subsequent sample fractions containing particles of different sizes can be collected. Importantly, the sample does not interact with a stationary phase which might degrade or alter the sample. AFFF is a robust and quick technique, needing only low-sample amounts. Further, the sample is reusable in other experiments.

For the AFFF fractionation, $10 \mu \mathrm{l}$ of $\mathrm{SV}$ stock solution at a protein concentration of $3.58 \mathrm{mg} / \mathrm{ml}$ is diluted in $40 \mu \mathrm{l}$ AFFF buffer $\left(150 \mathrm{mM} \mathrm{KCl}, 25 \mathrm{mM}\right.$ HEPES, $0.02 \% \mathrm{NaN}_{3}$ (sodium azide), $\mathrm{pH} 7.40$ ), giving a total final protein concentration of $0.72 \mathrm{mg} / \mathrm{ml} ; 30 \mu l$ of this solution is used for each AFFF run. An Eclipse 2 system from Wyatt Technology is used for the AFFF fractionation with a channel of height $350 \mu \mathrm{m}$ and length $275 \mathrm{~mm}$. A precut membrane of regenerated Cellulose (10 kDa molecular weight cut-off) is used. The Eclipse system is connected to a Agilant 1100 series HPLC pump, and to a Dawn Eos multi-angle light scattering setup. The channel flow is constantly $1.00 \mathrm{ml} / \mathrm{min}$. The sample is injected with an inject flow rate of $0.20 \mathrm{ml} / \mathrm{min}$ for $1 \mathrm{~min}$, and focused with a focus flow rate of $3 \mathrm{ml} / \mathrm{min}$ for $2 \mathrm{~min}$. Subsequently, the cross flow is set to $0.5 \mathrm{ml} / \mathrm{min}$ and is linearly decreased to $0 \mathrm{ml} / \mathrm{min}$ over $40 \mathrm{~min}$. Subsequent fractions are collected for $60 \mathrm{~s}$ each, starting 3 min after the injection of the SV sample, and 8 min after the initiation of the fractionation procedure. The total duration of one fractionation procedure is $40 \mathrm{~min}$.

\subsubsection{Instrumentation and Choice of Parameters}

If not indicated differently, the DLS measurments were performed at SVs dispered in HB100 buffer (100 mM KCl, 25 mM HEPES, 1 mM DTT, pH $7.4(\mathrm{KOH})$ ). For the DLS measurements of SV sample used for the AFFF fractionation, a SV stock solution of $3.58 \mu \mathrm{g} / \mu \mathrm{l}$ is diluted by a factor of 1000 with degassed aqueous AFFF buffer (150 mM KCl, $20 \mathrm{mM}$ HEPES, $0.02 \%$ $\mathrm{NaN}_{3}, \mathrm{pH} 7.4(\mathrm{KOH})$ ), leading to a total protein concentration of $3.58 \mu \mathrm{g} / \mathrm{ml}(28)$. Collected AFFF fractions are not further diluted for DLS measurements since the fractionation process already leads to a dilution factor of about 1000. The DLS measurement results are relatively insensitive to the exact particle concentration of SVs within a relatively large range (28). Effects 
due to the particle concentration on the DLS data have been ruled out by dilution series on a $\mathrm{SV}$ preparation with an initial protein concentration of $3.39 \mathrm{mg} / \mathrm{ml}$, corresponding to a vesicle concentration of about $(1.98 \pm 0.03) \times 10^{14}$ vesicles $/ \mathrm{ml}(14)$. For SV particle concentrations from $1.89 \times 10^{12}$ to $1.55 \times 10^{10}$ vesicles $/ \mathrm{ml}$ no significant effects on the auto-correlation function are visible after rescaling, and the DLS auto-correlation curves are highly reproducible for identical samples, see $(16,28)$.

The samples are put into cylindrical borosilicate cuvettes with a diameter of $10 \mathrm{~mm}$ (Fisher Scientific), and are closed air tightly with polymer caps (Carl Roth GmbH, Karlsruhe, Germany). DLS is performed with an ALV/CGS-3 Laser Light Scattering Goniometer System (ALV-GmbH, Langen, Germany), equipped with a $22 \mathrm{~mW}$ HeNe-Laser $(\lambda=632.8 \mathrm{~nm}$ from UNIPHASE, model 1145P), and an ALV-7004 or ALV-5004 Multiple Tau Digital Correlator. The scattered intensity is recorded by a high quantum efficiency avalanche photo diode at a scattering angle of $90^{\circ}$ in the scattering plane, perpendicular to the vertically polarized incident beam. If not indicated differently, the intensity auto correlation function calculated for three consecutive intervals of $30 \mathrm{~s}$ is averaged, corresponding to $90 \mathrm{~s}$ accumulation time. Three such runs are then performed to obtain the final averaged intensity auto correlation function $g_{2}(\tau)$, representing a total measurement time of $270 \mathrm{~s}$. The errors are calculated by the standard deviation of the three $90 \mathrm{~s}$ runs for each $\tau$. The resulting (normalized amplitude) correlation function $g_{1}(\tau)$ is given by $\beta\left|g_{1}(\tau)\right|=\sqrt{g_{2}(\tau)-1}(29,42)$ with the intensity correlation function $g_{2}(\tau)=<I(t) I(t+\tau)>_{t} /<I>_{t}^{2}$ and the coherence factor $\beta$.

\subsubsection{Results}

\section{Regularized inversion}

Figure $2.4(A)$ shows the size distribution functions of a selection of AFFF fractions. Fractions were collected every other minute over $60 \mathrm{~s}$ throughout the separation procedure. The first fraction shown was collected 5 min after injection of the SV sample (chronological order of fractions, with their time of collection [in minutes] after injection of the SV sample: red crosses [5], blue full squares [7], orange empty squares [9], magenta full triangles [11], purple full diamonds [13], light gray plus signs [15]).

The sample used for the AFFF corresponds to the same vesicle preparation depicted in Fig. 2.3. The size distribution functions were calculated by the ALV software and then rescaled to show the relative abundance of vesicles between the fractions. For each fraction the scaling factor $s$ was computed with the time averaged intensity $\langle I\rangle_{t}: s=\langle I\rangle_{t} / \sum_{R_{h}} p_{I}\left(R_{h}\right) d R_{h}$. In a final step, the distribution functions were normalized setting the peak height of the most abundant fraction to 1 . For comparison the size distribution of the unfractionated sample was included into the plot (solid black line). 



Figure 2.4: (A) Size distribution of SVs by DLS. Native SV preparation (solid black line) and individual AFFF fractionns (colored lines, time of collection [in minutes] after injection of the SV samples: red crosses [5], blue full squares [7], orange empty squares [9], magenta full triangles [11], purple full diamonds [13], light gray plus signs [15]) weighted by the relative number of particles within the corresponding individual AFFF fraction as determined from the time averaged scattering intensity $<I>_{t}$. The inset shows a schematic of the AFFF flow channel. Red arrows indicate the velocity $\mathrm{U}(\mathrm{x})$ of the buffer in direction of the flow channel, brown arrows indicate the direction of the channel cross-flow. (B) Size distribution of native SV preparation by DLS (solid black line) and cryo-EM (gray circles), shifted as detailed in the text, and individual AFFF fraction [5] from the shown native SV preparation (red crosses). 
The bulk of the synaptic vesicles in figure 2.4 (A) appears in the first fractions (red crosses [5], blue full squares [7]). These fractions contain purified vesicles, larger particles (>60 nm) are not present in any significant number, indicating the successful fractionation of SV away from larger particles. The difference between the peak position of the two size distribution functions obtained from fractions [5] and [7] is within the accuracy of the regularized fit. Later fractions only contain fewer SVs and some larger particles.

Figure 2.4 (B) shows a repetition from Fig. 2.3 of the size distribution function of the unfractionated sample measured by DLS (solid black line), the AFFF fraction [5], collected in minute 5 after injection of the SV sample, measured by DLS (red crosses [5]) and the size distribution of SVs in unfractionated samples determined by cryo-EM (grey circles). The cryoEM data on the radius of the SVs was corrected for the expected effects of the outer proteins on the hydrodynamic radius by adding $3 \mathrm{~nm}$.

The size distribution as determined by cryo-EM agrees well with the AFFF fraction [5]. The latter extends a little towards larger particles. On the other hand, the regularization method somewhat influences the shape of the distribution. Further, the distribution function of the unfractionated sample is also in good agreement with cryo-EM data and the single AFFF fraction data. Here, however, there are clearly a small number of larger particles present.

\section{Parameterized models}

Figure 2.5 (A) shows the intensity correlation functions $g_{2}(\tau)$ of a typical SV sample (green points) and of a typical individual AFFF fraction obtained from the native SV sample (black points), exhibiting the characteristic (exponential) decay expected for polydisperse colloidal particles undergoing Brownian motion, along with the errors estimated from the different runs and a least-square fit of a model as detailed below (solid red line and dashed red line). Fig. 2.5 (B) shows the resulting bimodal distribution function $p_{n}\left(R_{h}\right)$ of the synaptic vesicles (solid blue line, cryo-EM data, fixed during fitting) and of the larger aggregated membrane particles (dashed red line and solid red line). The cryo-EM data are taken from (15). Note the different scalings of the two components of $p_{n}\left(R_{h}\right)$, indicating that the large particles can be viewed as a small contamination.

The SV as well as the larger membranous fragments were modeled as spherical core-shell particles with a constant shell thickness of $t=12 \mathrm{~nm}$, accounting for both lipid and protein components of the structures. The lipid bilayer thickness is taken to be about $6 \mathrm{~nm}$ (15), and the contributions from the protein shells are taken into account by an additional $3 \mathrm{~nm}$ on both sides of the bilayer. In this way the small unilamellar vesicle structure of the SV and the membranous character of the larger particles are mimicked. The model form factor amplitude corresponding to this real space model is given in Eq. 2.3, above.

Using this model form factor amplitude, a forward calculation approach was implemented 

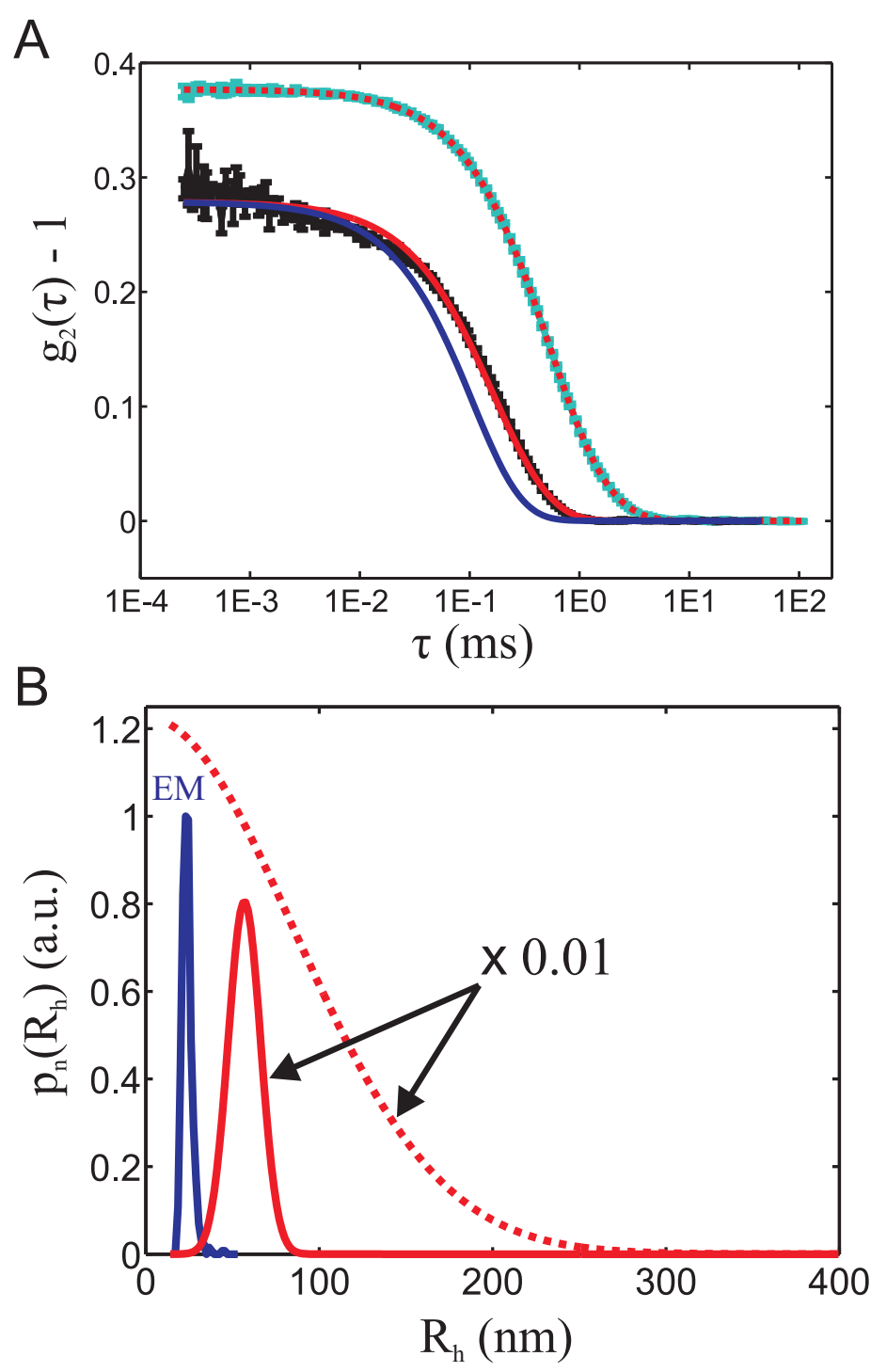

Figure 2.5: (A) DLS data of native SV ensemble (green dots) and AFFF fraction [5] (black dots), collected in minute 5 after injection of the SV sample, with error bars and fitted model calculations (red dashed line, red solid line). Calculated correlation function for particles following the cryo-EM size distribution (solid blue line). (B) Number weighted bimodal size distribution functions consisting of the blue branch (cryo-EM data, smoothed) and one of the red branches (fitted Gaussian distributions, solid red line, red dots) corresponding to the fits in $(A)$, (solid red line, red dots). Note the different scalings for the blue branch and the red branches. 
to analyze the SV data, circumventing problems usually arising from the standard inversion approach of $g_{1}(\tau)$ implemented in most commercial DLS instruments. For the calculations, the SV size distribution obtained by cryo-EM (smoothed) was used, shifted by $3 \mathrm{~nm}$ towards lager radii to account for the size increase of the hydrodynamic radius resulting from proteins facing outwards of the SV, which were not taken into account in the EM size measurement.

The contribution from the larger membranous particles was parametrized with a Gaussian shaped size distribution. Together with the main population of isolated and intact SVs this second component forms a bimodal size distribution. Baselines for $\tau \rightarrow 0$ and $\tau \rightarrow \infty$ were fitted to $g_{2}(\tau) . p_{n}\left(R_{h}\right)$ was optimized by a least-squares fit to $g_{2}(\tau)$, solely by adjusting the position, width and relative height of the Gaussian size distribution of the larger particles to the SV population. The size distribution and relative contribution of the larger membranous particles depend to some extent on the individual SV preparation. The resulting calculated auto correlation curves are found to be in excellent agreement with the measured $g_{2}(\tau)$ for the native SV ensemble (reduced $\chi^{2}=1.36$ ), and in fair agreement for the individual AFFF fraction (reduced $\chi^{2}=10.92$ ).

\subsubsection{Discussion and Conclusions}

Regularized nonlinear inversion of the intensity correlation function by the ALV software (Fig. 2.3) gives access to the intensity weighted size distribution function $p_{I}\left(R_{h}\right)$, or number weighted size distribution function $p_{n}\left(R_{h}\right)$ of the SV population. Width and shape depend to some extent on the regularization. However, the maximum of $p_{n}\left(R_{h}\right)$ is hardly affected by different regularization parameters. Due to imperfections in the consideration of the form factor model, a second peak at a radius of about $160 \mathrm{~nm}$ is present in $p_{n}\left(R_{h}\right)$. This peak occurs in all cases, where $p_{I}\left(R_{h}\right) \neq 0$ for $F\left(q, R_{h}\right)=0$ and is more pronounced in cases where the particles are modeled as hollow hard spheres as compared to hard spheres (see below). A precise assessment of the relative particle number of sizes within the range of the artificial peak is omitted here.

The size distributions as obtained from the DLS data are found to depend critically on the purity of the investigated SV samples. Especially even relatively few larger contaminant particles influence the obtained size distributions considerably, owing to the highly nonlinear dependence of the number of photons scattered at particles of different sizes.

The number of larger contaminant particles in the SV samples with different diffusion properties than SVs are significantly reduced by AFFF which greatly decreases the contribution of the tail towards larger radii in $p_{n}\left(R_{h}\right)$ (solid black line and red crosses in Fig. 2.4). The size distribution of SV samples after purification by AFFF can consistently be obtained by cryo-EM and inversion of regularized DLS data (gray circles and red crosses in Fig. $2.4(B)$ ). Results are in excellent agreement with values reported previously (14). 
Thus, the AFFF separation process is found to discriminate well between SV and larger trace particles and is suited for further purification of SV samples. In the course of the AFFF fractionation process, the sample is diluted here by a factor of about 1000. Depending on separation parameters it seems feasible to sub-fractionate the actual SV population, giving access to SV sub-populations precisely defined and selected by their diffusion properties. However, the size distribution obtained by DLS extends still somewhat further towards larger radii (red crosses in Fig. $2.4(B)$ ), or a very small additional contribution of somewhat larger particles is needed (solid red line in Fig. 2.5) when compared to the cryo-EM size data. This may be due to an underestimation of larger SV particles by cryo-EM due to under sampling, and reflects the effects of very few remaining larger particles in the sample. In the case of the inversion analysis, it cannot be excluded, that part of the small deviation between the size distribution obtained by cryo-EM when compared to the one obtained by DLS is also due to regularization effects.

Scattering techniques allow here to obtain information averaged over a fairly large number of particles as compared to single particle imaging techniques like cryo-EM in reasonably short times (here in the order of $100 \mathrm{~s}$ ). It seems feasible to significantly increase time resolution reaching values in the order of a few seconds. The needed sample volumes are relatively small (about $1 \mu \mathrm{g}$ of SVs in about $1 \mathrm{ml}$ buffer) and samples are reusable.

In summary, DLS spectra recorded from SV under quasi-physiological conditions can be consistently described with a size distribution obtained by cryo-EM at vitrified SV dispersions. The effects originating from few larger trace particles of sizes in the order of few $100 \mathrm{~nm}$ in samples can be effectively modeled by a second Gaussian distributed branch in the particle size distribution.

Regularization and inversion of DLS spectra from samples containing relatively few of such larger trace particles lead to a mono-modal size distribution with a significantly overestimated width when compared to the SV size distribution obtained by cryo-EM. The actual SV size distribution is rather sharp, compared to the rather broad size distribution of the larger membranous particles. Although the position of the maximum of the size distribution is also slightly shifted towards larger radii, it is found to be still a fairly good estimate for the actual most likely radius within the SV population.

Larger trace particles contaminating the SV population can be removed effectively by AFFF fractionation, giving access to diluted SV dispersions of utmost purity. DLS spectra from AFFF fractions containing the SV population can be analyzed by means of regularization and inversion and reveal a SV size distribution consistent with cryo-EM data.

DLS is found to be a fast and reliable method to obtain information on the ensemble averaged size and size distribution of dispersed synaptic vesicles within the range of approximately $10 \mathrm{~nm}$ to few $100 \mathrm{~nm}$. Short measurement times and small sample amounts needed for DLS allow to 
investigate large numbers of samples in a batch. No invasive sample preparation steps are needed and DLS measurements are compatible with quasi-physiological conditions. Samples are reusable after DLS measurements.

\subsection{Sample Preparation for X-Ray Scattering Experiments}

\subsubsection{Native Synaptic Vesicles}

Synaptic vesicles from rat brain were purified by Matthew Holt ${ }^{6}$ following the purification protocol by Jahn et al., (14), as described in section 2.3 above. Samples were kept on water/ice mixtures until investigation by electron microscopy (EM). Samples for the SAXS measurements were snap frozen for transportation to the synchrotron. In similar samples, the V-ATPase on SVs retained the capacity to acidify the interior of the SV in the presence of ATP (build-up of a proton gradient, as assayed by the quench of the fluorescent dye acridine orange as an indicator), which is an important indication that the SV membranes are functionally intact (43).

The resulting SV stock solutions had a protein concentration in the range of $5.47-6.45 \mu \mathrm{g} / \mu \mathrm{l}$, which were partly further diluted by adding HB100 buffer at pH 7.40.

Samples at low pH were prepared by adding HB100 buffer ajusted to pH 2.10 to the SV stock solution in a ratio of 4:1. Correspondingly, samples at high $\mathrm{pH}$ were prepared by adding HB100 buffer at pH 12.90 to the SV stock solution in a ratio of 4:1.

\subsubsection{Protease Treatment Synaptic Vesicles}

For the protease digested SVs (44), one sample was split in two following the usual purification. To one sample $0.1 \mu \mathrm{g}$ Trypsin per $\mu \mathrm{g}$ protein was added and incubated at $37^{\circ} \mathrm{C}$ for 60 minutes. The other sample was incubated for the same time on ice as a control. Both samples were centrifuged at $3 \times 10^{5} \mathrm{~g}$ (average) for two hours. The pellets were then re-suspended in aqueous buffer of $100 \mathrm{mM} \mathrm{KCl,} 25 \mathrm{mM}$ HEPES and $1 \mathrm{mM}$ DTT at $\mathrm{pH}$ 7.40. Both the centrifugation and re-suspension followed similar steps of the usual purification protocol.

\subsubsection{Preparation of Liposomes}

Small unilamellar lipid vesicles were prepared by dissolving 1,2-Dioleoyl-sn-Glycero-3-Phosphatidilserine (DOPS) supplied by Avanti Polar Lipids (Alabaster, AL) at a concentration $c=$ $20 \mathrm{mg} / \mathrm{ml}$ in ultrapure water (Milli-Q), and sonication to clarity with a Sonoplus tip sonicator (Bandelic electronic, Berlin, Germany), set to $45 \%$ power and 6 cycles. Samples were

\footnotetext{
${ }^{6}$ Department of Neurobiology, Max Planck Institut für Biophysikalische Chemie, Göttingen, Germany
} 
centrifuged for $10 \mathrm{~min}$. at $14.5 \times 10^{3} \mathrm{rpm}$ to remove metal particles originating from the sonotrode, and subsequently degassed at room temperature for $10 \mathrm{~min}$ in a Heraeus vacutherm VT 6060P vacuum oven (Kendro Laboratory Producs, Hanau, Germany), operated with a diaphragm vacuum pump (Balzers-Pfeiffer, Asslar, Germany).

\subsection{Conclusions}

SVs can be purified in sufficient large quantities and purity from rat brain needed for synchrotron SXAS experiments. The purity of the SV dispersions can be assessed by employing cryo-EM and DLS. Shape information is obtained for both the SVs and the larger trace particles by cryo-EM. The size distribution of SVs is independently obtained by cryo-EM and DLS. Larger trace particles present in the SV dispersions can be identified by cryo-EM, and quantified by DLS. 


\section{Chapter 3}

\section{Small-Angle X-Ray Scattering}

This chapter introduces basic experimental aspects of SAXS, including raw data treatment, and presents measured scattering curves. The experimental set-ups and parameters used in the SAXS measurements are given (Section 3.1). SAXS curves from native synaptic vesicle (Section 3.2), recorded independently at two different synchrotrons, are compared revealing high reproducibility of data taken from different individual samples and at different experimental set-ups. A dilution series reveals the absence of a pronounced interaction potential between the SVs. SAXS curves from native SVs measured at three different $\mathrm{pH}$ values show distinct differences in their scattering curves. Further, SAXS curves obtained from protease treated SVs show distinct features when compared to native SVs. Finally, SAXS curves from unilamellar liposomes are compared to data from SVs. Model independent conclusions are drawn from the scattering data (Section 3.3).

\subsection{Experiments and Instrumental Corrections}

Figure 3.1 shows a schematic of the experimental set up for the SAXS measurements. The momentum transfer vector is defined by $\mathbf{q}=\mathbf{K}_{f}-\mathbf{K}_{i}$, where $\mathbf{K}_{f}$ and $\mathbf{K}_{i}$ are the wave vectors of the scattered and incident x-ray beam, respectively. The modulus of the scattering vector $\mathbf{q}$ is given by

$$
q \equiv|\mathbf{q}|=\frac{4 \pi}{\lambda} \sin \Theta
$$

where $\lambda$ is the wave length of the incident photons, as well as of the elastically scattered photons. Photons are scattered to angles $2 \Theta$ relative to the incident beam, see Fig. 3.1.

SAXS experiments were performed at the high brilliance undulator beamline ID-2 of the European Synchrotron Radiation Facility (ESRF) in Grenoble, France, and beamline B1 at the Doris III storage ring of HASYLAB at Deutsches Elektronen-Synchrotron, Hamburg, Germany. 




Figure 3.1: Schematic of experimental setup for SAXS measurements. The momentum transfer vector is defined by $\mathbf{q}=\mathbf{K}_{f}-\mathbf{K}_{i}$, where $\mathbf{K}_{f}$ and $\mathbf{K}_{i}$ are the wave vectors of the scattered and incident $\mathrm{x}$-ray beam, respectively.

\subsubsection{Beamline ID-2, ESRF}

Beamline ID-2 was operated under the beamline's standard conditions at $E=12.4 \mathrm{keV}$ (45) photon energy. The beamline is operated with a monochromatic, highly collimated and intense beam in a pinhole configuration. The cryogenically cooled Si-111 double-crystal monochromator (bandwith in the order of $\Delta E / E=2 \times 10^{-4}$ for $E=12.4 \mathrm{keV}$ ) displaces the beam vertically by $30 \mathrm{~mm}$ to reduce the background radiation from the electron storage ring. An uncooled Rh-coated double-focusing toroidal mirror follows the monochromator and minimizes the beam size at the detector position for the longest possible source to detector distance at the beamline $(65 \mathrm{~m})$. Beam size is in the order of $100 \mu \mathrm{m}$.

The SV samples were at total protein concentrations of $2.74 \mu \mathrm{g} / \mu \mathrm{l}$ in aqueous buffer of $100 \mathrm{mM} \mathrm{KCl}, 25 \mathrm{mM}$ HEPES and $1 \mathrm{mM}$ DTT at pH 7.40. The samples were kept in a glass flow through capillary, or wax sealed glass capillary, with a diameter of $1.5 \mathrm{~mm}$ and a wall thickness of $0.01 \mathrm{~mm}$.

The diffraction patterns were recorded with a fiber optically coupled FReLoN ${ }^{1}$ CCD detector positioned 0.85 and $5 \mathrm{~m}$ behind the sample in an evacuated detector tube. The $\mathrm{CCD}^{2}$ has an input field of $100 \mathrm{~mm} \times 100 \mathrm{~mm}$ and a nominal dynamic range of $16 \mathrm{bit}$, (15 bit above the noise floor). The spatial resolution, as determined by the point spread function, is about $80 \mu \mathrm{m}$. The detector was protected from the primary beam by a beam stop of size $2.5 \times 6 \mathrm{~mm}$, equipped with a PIN photo diode for measurements of the primary beam intensity after being attenuated by the sample. Data were collected over a $q$-range from 0.016 to $5.5 \mathrm{~nm}^{-1}$. Typical exposure

\footnotetext{
${ }^{1}$ Fast-Readout, Low-Noise

${ }^{2}$ Kodak KAF-4320, Eastman Kodak Company
} 
times were 0.1 seconds.

The number of pixels was reduced by $2 \times 2$ binning from $2048 \times 2048$ to $1024 \times 1024$ virtual pixels. The $2 \mathrm{D}$ isotropic (powder average) diffraction pattern was corrected for the CCD dark current, offset of the analogue to digital converter, spacial distortion, detector sensitivity (flat-field), and was calibrated to an absolute scale (water reference) employing the procedure described in (45). The SAXS utilities ${ }^{3}$ program package from Michael Sztucki $^{4}$ has been employed for these on-line data processing steps.

Detector pixels with very low count rates close to the edge of the detector as well as in the regions of the shadow of the beam stop were masked and excluded from the further data processing. The mask is generated using the program Fit2D ${ }^{5}$, and saved as a $B S L$ file, which is converted to a $E D F$ file by employing the script file mask2b2.mac ${ }^{6}$ from Michael Sztucki, as described in more detail in the diploma thesis of Gudrun Lotze (46). The file names of the masks employed for processing the scattering data recorded at the different sample to detector distances are (file name, distance in m): (A99000.BSL, 2.00), (B99000.BSL, 5.00), (C99000.BSL, 0.85).

Figure 3.2 (A) shows the centrosymmetric scattering pattern from dispersed SVs, recorded with a sample to detector distance of $5 \mathrm{~m}$. The data is re-binned and corrected for the CCD dark current, the offset of the analogue to digital converter, spacial distortion and the detector sensitivity. (B) shows the same scattering pattern as in (A), masked pixels are marked (black), and excluded from the further data processing.

The corrected, calibrated and masked scattering pattern is azimuthally regrouped, and subsequently azimuthally averaged, by employing the script file ccdmulticalib_carianz_trm.mac $^{7}$ from Michael Sztucki, which requires and uses functions from the SAXS program package ${ }^{8}$ from Peter Bösecke ${ }^{9}$. The output of the script file is written in DAT files, containing one header line and three columns with entries giving the values of the modulus of the scattering vector $q$ in units of $(1 / \mathrm{nm})$, the recorded scattering intensities $I(q)$ in dimensionless units (1), and the corresponding counting error statistics, neglecting correlations between pixels. In order to obtain the scattering intensity on an absolute scale in units of an inverse length, $I(q)$ is divided by the sample thickness in units of the corresponding length in real space. Further, $I(q)$ is multiplied by a dimensionless correction factor with a value of approximately 1.5 which is empirically determined from the absolute scattering intensity of water at small q-values, and accounts for all processes which reduce the number of scattered photons reaching the detector.

\footnotetext{
${ }^{3}$ http://www.sztucki.de/SAXSutilities/

${ }^{4}$ European Synchrotron Radiation Facility, High Brilliance Beamline ID-2, Grenoble, France

${ }^{5}$ http://www.esrf.eu/computing/scientific/FIT2D/

${ }^{6}$ http://www.sztucki.de

${ }^{7}$ http://www.sztucki.de

${ }^{8}$ http://www.esrf.eu/UsersAndScience/Experiments/TBS/SciSoft/OurSoftware/SAXS

${ }^{9}$ European Synchrotron Radiation Facility, High Brilliance Beamline ID-2, Grenoble, France
} 

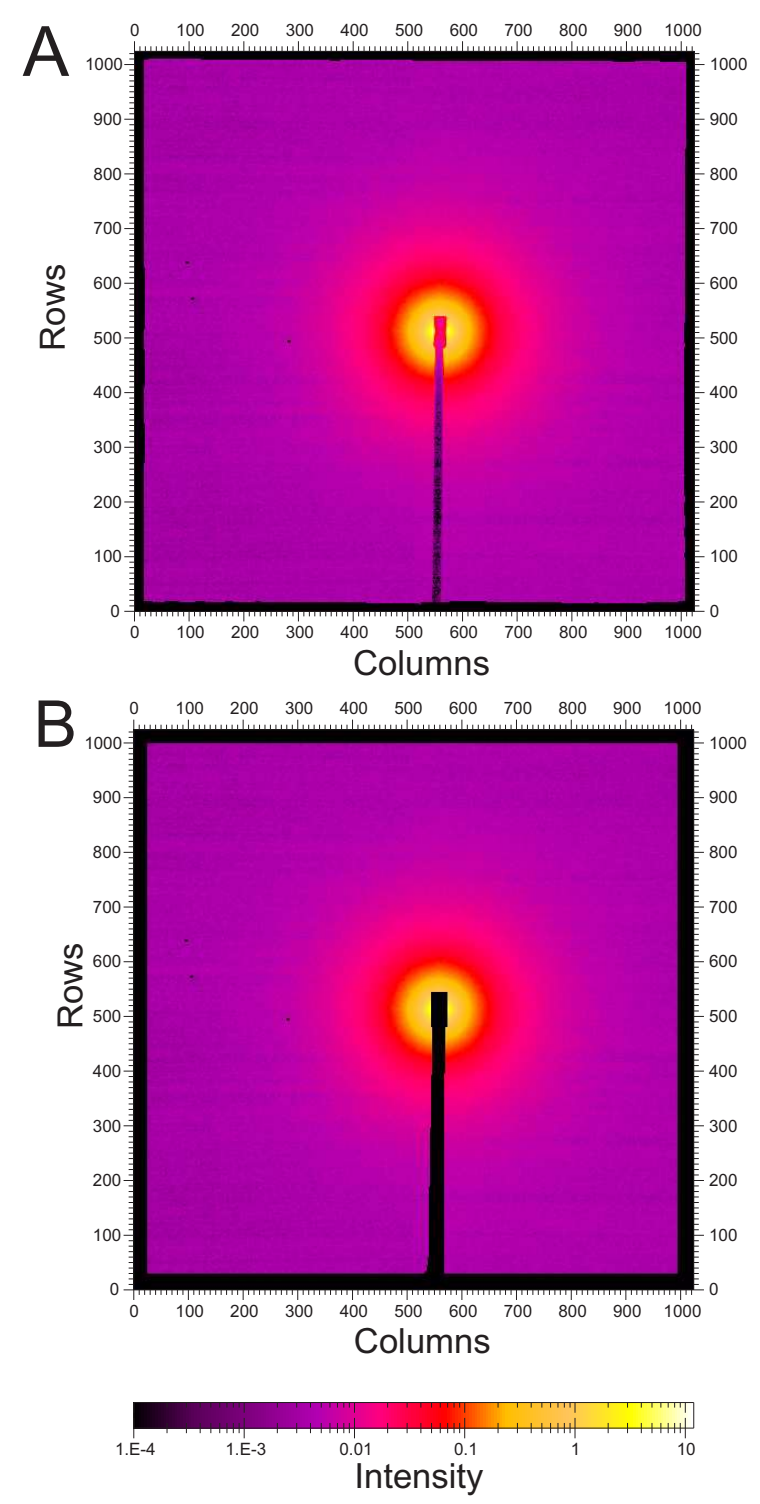

Figure 3.2: Diffraction pattern from dispersed native SVs recorded at a distance of $5 \mathrm{~m}$ behind the sample (Beamline ID-2, ESRF). (A) Data re-binned, corrected for CCD dark current, the offset of the analogue to digital converter, spacial distortion and detector sensitivity, as detailed in the text. (B) Pixels at the detector edge and in the region of the beam stop shadow are masked (black) and excluded from the further data processing. 




Figure 3.3: Scattering curves recorded from a SV dispersion (green diamonds), and pure HB100 buffer (blue triangles) in a flow through glass capillary at ID-2, ESRF. The scattering curve from the buffer sample is subtracted from the scattering curve from the SV sample, yielding the final corrected scattering curve from the SV (black circles, shifted by a factor of 0.1 , for clarity) used for fitting.

Data recorded at the two different detector distances are manually combined in MATLAB giving a corrected scattering curve $I(q)$ covering more than two orders of magnitude in $q$. Further, the scattering data of the vesicles is corrected for the scattering of the aqueous buffer, and the thin glass capillary, by subtracting the scattering curve recorded from a capillary filled with pure buffer, from the scattering curve as recorded of the vesicle dispersions, yielding the final $1 \mathrm{D}$ scattering curve for fitting. Figure 3.3 shows the scattering curves recorded from a SV dispersion (green diamonds), and from pure HB100 buffer (blue triangles) in the flow through capillary. The scattering of the buffer is slightly rescaled by a factor close to 1 (if necessary) to account for imperfections in the calibration procedures of the scattering intensities to an absolute scale. The scattering curve from the buffer is subtracted from the scattering curve of the SVs, yielding the final corrected scattering curve (black circles, shifted by a factor of 0.1 , for clarity) used for fitting and model falsification.

Radiation damage was ruled out by comparison of scattering patterns recorded with different exposure times from 0.01 to 10 seconds. For the standard accumulation time, the absorbed dose during exposure was about $6.5 \times 10^{3} \mathrm{~Gy}$, as calculated from the number of photons impinging on the sample, and the absorption of the sample. Dilution series revealed no measurable inter-particle correlations or aggregation for samples of a total protein concentration between $6.45 \mu \mathrm{g} / \mu \mathrm{l}$ and $0.10 \mu \mathrm{g} / \mu \mathrm{l}$, see section 3.2. Detector resolution effects could be ignored at the 
ID-2, ESRF, measurements.

\subsubsection{Beamline B1, HASYLAB}

Beamline B1 was operated under the beamline's standard conditions at $9.0 \mathrm{keV}$ (47) photon energy. The beamline operates in a nonfocusing pinhole collimation geometry. The x-ray photons are generated by employing a bending magnet (critcal energy $16.04 \mathrm{keV}$ ), which are monochromatized by a fixed exit monochromator consisting of two flat symetrically cut Si311 crystals. The higher harmonics are suppressed by detuning the second monochromator crystal (MOSTAB unit (48)). The beam size at the sample was $1.0 \times 0.7 \mathrm{~mm}$ (horizontally $\times$ vertically), defined by the guard slits in front of the sample.

The SV samples were at total protein concentrations of $4.98 \mu \mathrm{g} / \mu \mathrm{l}$ in aqueous buffer of $150 \mathrm{mM} \mathrm{KCl}, 25 \mathrm{mM}$ HEPES and $1 \mathrm{mM}$ DTT at pH 7.40. The samples were kept in wax sealed glass capillaries with diameters of $1.5 \mathrm{~mm}$ and a wall thicknesses of $0.01 \mathrm{~mm}$.

The diffraction patterns were recorded with a multiwire proportional chamber gas detector (Gabriel), or a Pilatus 300k silicon single photon counting pixel array detector positioned 935 and $3635 \mathrm{~mm}$ behind the sample. The pixel sizes of the detectors are $0.8 \mathrm{~mm}$ (Gabriel), and $172 \mu \mathrm{m}$ (Pilatus). Data were collected over a $q$-range from 0.08 to $2.9 \mathrm{~nm}^{-1}$. Typical exposure times were 30 minutes. The 2D isotropic (powder average) diffraction pattern was corrected for the dark current, fluctuations in primary intensity, spacial distortion, detector sensitivity / flat-field (only for Gabriel detector), and was calibrated to an absolute scale (glassy carbon reference) employing a similar procedure as described in (45). Pixels in the shadow of the beam stop, at the very edge of the detector, and in regions with significant scattering from slits were masked and excluded from the further data analysis. The scattering pattern were azimuthally integrated, and data recorded at the two different detector distances were combined to give a corrected scattering curve $I(q)$.

All instrumental corrections were performed using a data processing toolbox ${ }^{10}$ consisting of various individual MATLAB macros developed by Ulla Vainio ${ }^{11}$, A. Wacha and S. Haas. The macros utilized in the processing of the data recorded with the Gabriel ${ }^{12}$ detector, and the Pilatus ${ }^{13}$ detector are distributed by Ulla Vainio. More details on the functionality of individual macros of the data processing toolbox can be found in the beamline manuals ${ }^{14}$ by Ulla Vainio. Note that the macros for the Pilatus detector require the macro imageread. ${ }^{15}$, available from

\footnotetext{
${ }^{10}$ http://hasylab.desy.de/facilities/doris_iii/beamlines/b1/software_and_hardware/index_eng.html

${ }^{11}$ Hamburger Synchrotronstrahlungslabor at Deutsches Elektronen-Synchrotron, Beamline B1, Hamburg, Germany

${ }^{12} \mathrm{http}: / /$ github.com/uvainio/B1macrosGabriel/

${ }^{13}$ http://github.com/uvainio/Beamline-B1-macros/

${ }^{14}$ http://hasylab.desy.de/facilities/doris_iii/beamlines/b1/software_and_hardware/index_eng.html

${ }^{15}$ http://sls.web.psi.ch/view.php/beamlines/ms/sd/computing/index.html
} 
the Swiss Light Source.

The data processing toolbox writes several output files. Files named intnorm*.dat contains three columns with entries giving the values of the modulus of the scattering vector $q$ in units of $(1 / \AA)$, the recorded scattering intensities $I(q)$ in units of $(1 / \mathrm{cm})$, and the corresponding counting error statistics, neglecting correlations between pixels. The sample thickness in units of $(\mathrm{cm})$ is determined manually and entered prior to the start of the data processing into the macro B1normint*.m, which is used for the normalization of the data to an absolute scale. The file named intnorm*.log contains different experimental parameters, such as beam size and calibrated energy. The file contains re-binned data, so that scattering data recorded at different sample to detector distances are sampled at equal $q$ values. The data structure and units in the file intbinned*. dat are the same as in file intnorm* ${ }^{*}$.dat. Data recorded at different detector distances are combined by employing the macro sumanduniteB1*.m,

Further, the scattering data from the vesicles is corrected for the scattering from the aqueous buffer, and the thin glass capillary, by subtracting the scattering curve recorded from a capillary filled with pure buffer, from the scattering curve as recorded from the vesicle dispersions, yielding the final 1D scattering curve for fitting. The scattering curve from the buffer is slightly rescaled by a factor close to 1 (if necessary) to account for imperfections in the calibration procedures of the scattering intensities to an absolute scale. The scattering curve from the buffer is subtracted from the scattering curve of the SVs in MATLAB, yielding the final corrected scattering curve used for fitting and model falsification. Detector resolution effects could be ignored at the B1, HASYLAB, measurements.

\subsection{Results}

Figure 3.4 shows SAXS data from native SVs recorded at beamline ID-2 at ESRF (black circles) and at beamline B1 at HASYLAB (green squares). Plotted are (A) the scattered intensity $\mathrm{I}(\mathrm{q})$ vs. modulus of the scattering vector $q$, and $(B)$ the $\mathrm{I}(\mathrm{q}) \times q^{2}$ vs. $\mathrm{q}$ (Kratky plot). Data has been background corrected, radially integrated and calibrated to an absolute scale following the procedures described in section 3.1. Note that the two data sets have been recorded from samples from different individual SV preparations with similar protein concentrations, and at two different experimental set-ups. Both the data sets look very similar, only towards low $q$ values there are small, but significant differences in the slope of the curves. This is most likely due to small differences in the number of larger trace particles originating from imperfections in the SV purification procedure, see sections 2.3 and 2.4 .

Figure 3.5 shows a dilution series of native SVs. SV sample concentrations are $6.45 \mu \mathrm{g} / \mu \mathrm{l}$ (black circles), $1.08 \mu \mathrm{g} / \mu \mathrm{l}$ (blue squares), and $0.10 \mu \mathrm{g} / \mu \mathrm{l}$ (green diamonds). The three scattering curves exhibit very similar slopes when compared to each other at similar q values. As 
A



B

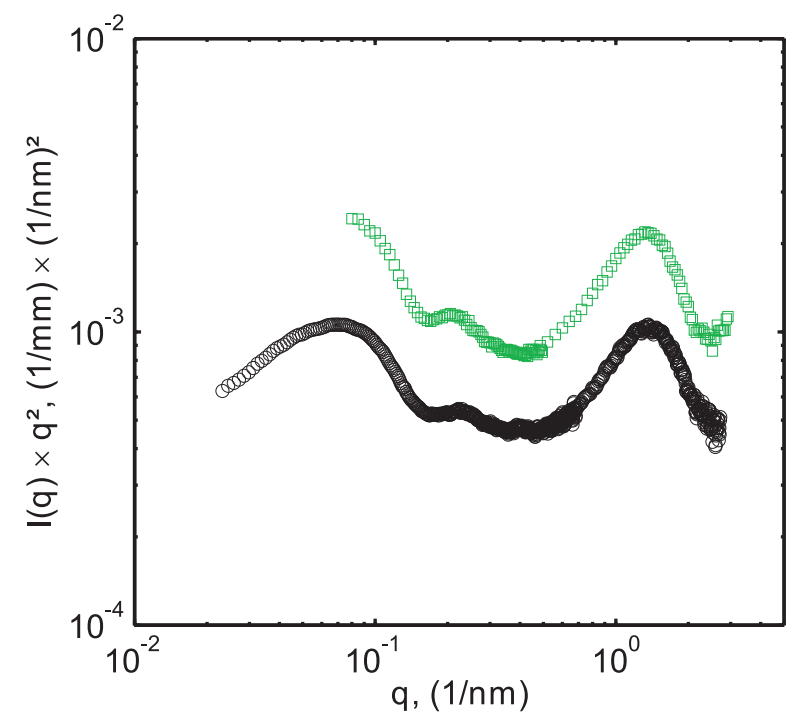

Figure 3.4: SAXS data from native SVs recorded at beamline ID-2 at ESRF (black circles) and beamline B1 at HASYLAB (green squares, shifted for clarity). (A) Plot of the scattered intensity $\mathrm{I}(\mathrm{q})$ vs. the modulus of the scattering vector $\mathrm{q}$, and $(B)$ Kratky plot, i.e. $\mathrm{I}(\mathrm{q}) \times q^{2}$ vs. q. Data has been background corrected, radially integrated and calibrated to an absolute scale, as detailed in the text. Exposure time $0.1 \mathrm{~s}$ (ID-2, ESRF), and 30 min (B1, HASYLAB).

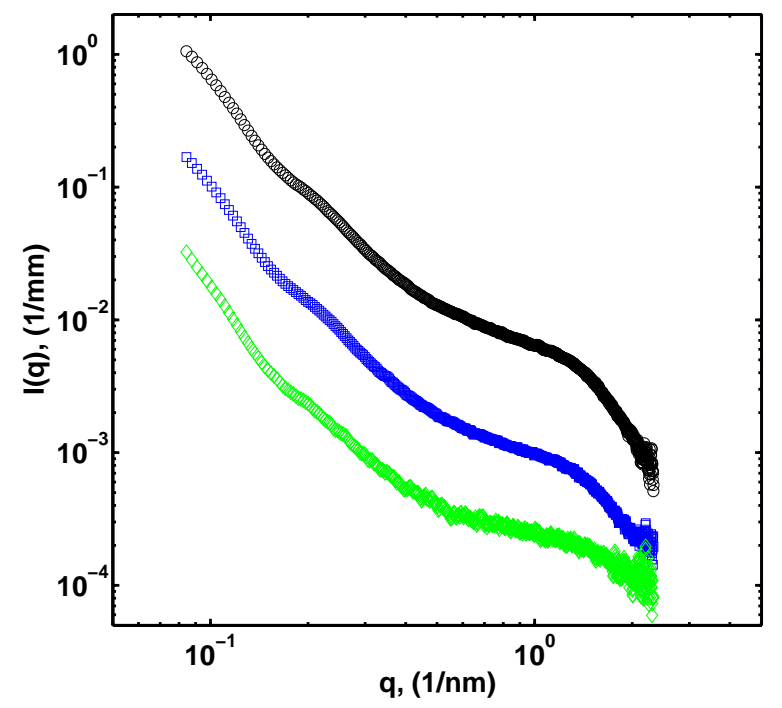

Figure 3.5: SAXS data from dilution series of native SVs recorded at beamline ID-2 at ESRF. SV sample concentrations are $6.45 \mu \mathrm{g} / \mu \mathrm{l}$ (black circles), $1.08 \mu \mathrm{g} / \mu \mathrm{l}$ (blue squares), and $0.10 \mu \mathrm{g} / \mu \mathrm{l}$ (green diamonds). 


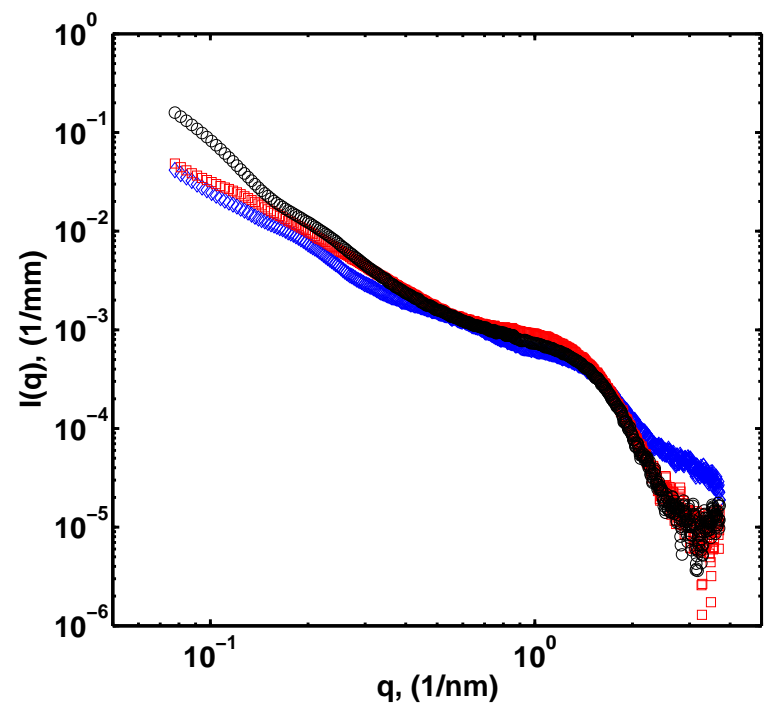

Figure 3.6: SAXS data from native SVs under different $\mathrm{pH}$ conditions, recorded at beamline ID-2 at ESRF. SVs at pH 7.40 (black circles), SVs at low (2.1) pH (blue diamonds), and SVs at high (12.9) $\mathrm{pH}$ (red squares). Figure adapted from (17).

expected, the curves are vertically shifted due to the different sample concentrations as I(q) has been calibrated to an absolute scale. Note the differences between the theoretically expected shifts due to the sample concentration, and the experimentally observed intensities. The differences are due to imperfections and uncertainties in the calibration procedure of the data to an absolute scale. There are no indications of a pronounced interaction potential between the SVs in the samples. Thus, the structure factor can be approximated by 1 within the investigated $\mathrm{q}$ range.

Figure 3.6 shows data from SV samples in HB100 buffer at pH 2.1, pH 7.4 and pH 12.9. The SAXS curves show distinct features depending on the $\mathrm{pH}$ conditions. The overall slope of the scattering curves is decreased at both high and low $\mathrm{pH}$ conditions as compared to neutral pH. This might be due to changes in overall shape and/or size of the SVs. Also aggregation (docking) of SVs might lead to these effects. Resolved changes in the higher $q$ regions of the recorded data indicate distinct changes in the local structure of the protein decorated bilayer of the SVs as a function of $\mathrm{pH}$ conditions. The underlying detailed structural changes are to be further investigated and remain unclear at present. However, the SVs seem to retain well defined structures even under extreme $\mathrm{pH}$ conditions. More subtle changes of the chemical environment of the SV samples like different calcium concentrations in the range of 0 to $100 \mu \mathrm{M}$, or magnesium concentrations around $200 \mathrm{mM}$ did not show resolvable differences in the corresponding SAXS curves (data not shown).

Figure 3.7 shows the SAXS intensity function $(A) I(q)$ vs. $q$, and $(B)$ a Kratky plot $I(q) \times q^{2}$ vs. $q$ for a SV sample (black circles) and SV treated with the protease Trypsin (blue squares). 
A

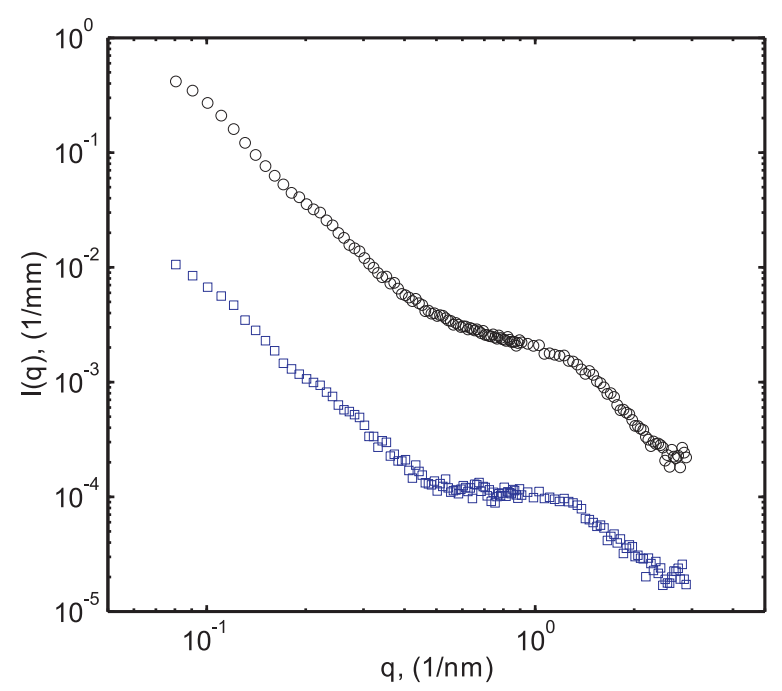

B

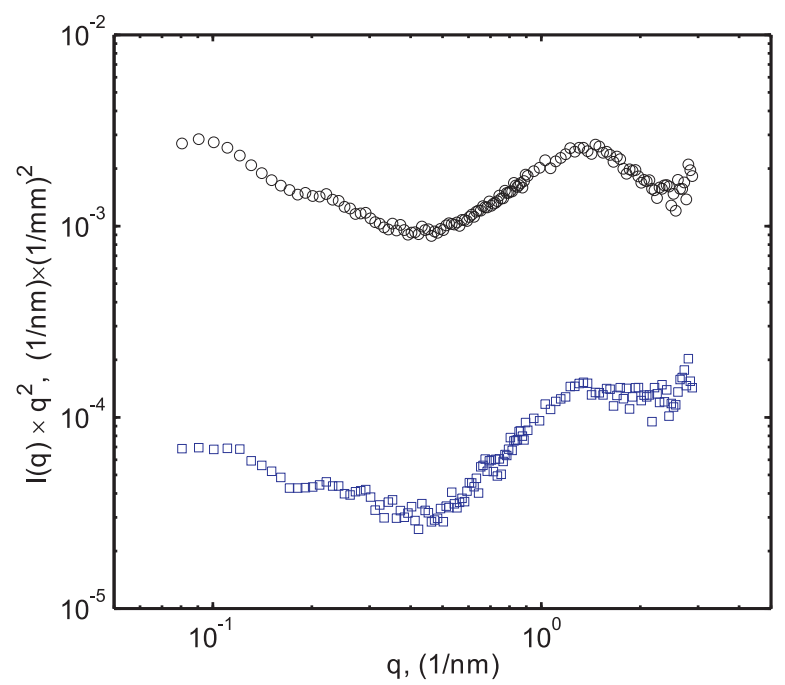

Figure 3.7: SVs with additional centrifugation in purification protocol (black circles), and SVs treated with protease to remove protein residues facing outwards, also with an additional centrifugation in purification protocol (blue squares, shifted by factor 0.1 for clarity). (A) $I(q)$ vs. $q$, and $(B)$ Kratky plot $I(q) \times q^{2}$ vs. $q$. Figure partly adapted from (17).

Clearly visible are distinct, significant differences between the two scattering curves. The scattering curve from the protease treated SVs (blue squares) exhibits a decreased slope for $q \approx 0.5$ to $q \approx 11 / \mathrm{nm}$ when compared to the control sample (black circles).

Figure 3.8 shows data $I(q)$ vs $q$ from a control sample consisting of DOPS vesicles, $20 \mathrm{mg} / \mathrm{ml}$ in water (blue squares), and how they compare to SV data (black circles). While the data looks qualitatively similar towards both lower and higher q values for the measured interval of the DOPS vesicle data, the scattered intensities differ up to approximately two orders of magnitude at intermediate $q$-values.

\subsection{Conclusions}

SAXS measurements from dispersed SVs under quasi-physiological conditions reveal characteristic and distinct isotropic scattering patterns. The data is highly reproducible at different synchrotron beamlines, and depends only for smaller q values slightly on the individual SV purification. This is due to a slightly varying small number of larger trace particles in the sample, due to unavoidable imperfections in the SV purification protocol, see section 2.3. A dilution series reveals no significant inter-particle potential for SVs dispersed in HB100 buffer at $\mathrm{pH}$ 7.4. Thus, the structure factor can be approximated by 1 in the measured $\mathrm{q}$ interval under neutral pH conditions. The recorded SAXS curves from SVs under different pH condi- 


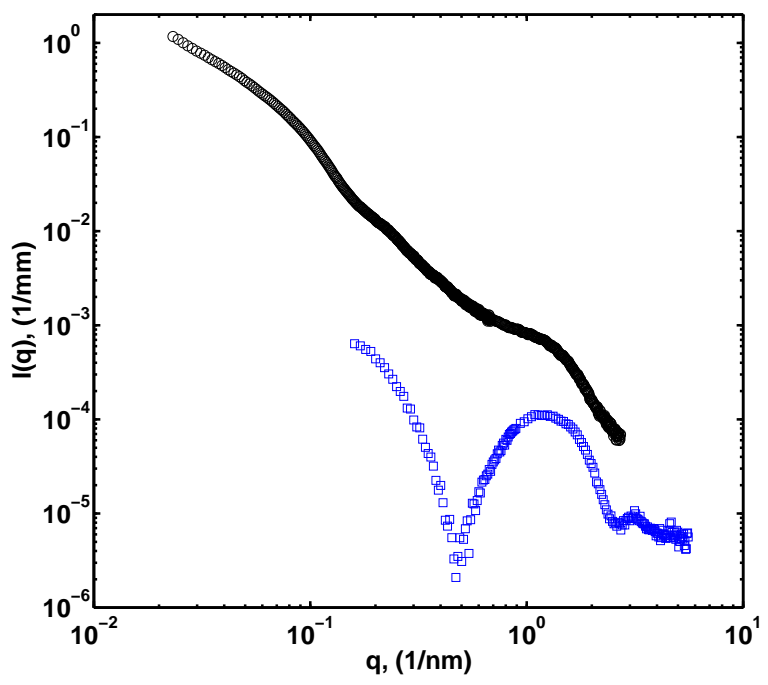

Figure 3.8: DOPS vesicle data (blue squares, $\times 10^{-3}$ ) recorded at beamline B1 at HASYLAB, and SV data (black circles) recorded at beamline ID-2 at ESRF. SV data identical to data shown in Fig. 3.4. Adapted from (17).

tions show distinct, characteristic changes when compared to each other. Thus, SAXS is suited to follow structural changes in the scattering patterns from SVs induced by variation of the quasi-physiological environment. Scattering curves from SVs treated with a protease (Trypsin) show small, but significant characteristic differences when compared to scattering curves from a control sample consisting of native SVs. The scattering curves recorded from unilamellar DOPS vesicles, when compared to SV data, show well distinct features in intermediate q ranges of the measured q regions, while they exhibit qualitatively similar scattering curves both towards higher and lower q values. 


\section{Chapter 4}

\section{Data Modeling of X-Ray Scattering}

\section{Curves}

This chapter introduces basic theoretical scattering models and describes approaches for the analysis of data from SVs. The description of kinematic photon scattering at the electrons of polydisperse core-shell particles within the continuum approximation is introduced, and the calibration of the scattering data from SVs to an absolute scale is addressed (Section 4.1). We consider approaches of model independent analysis of SAXS data and their limitations (Section 4.2 ), and introduce the concept of direct modeling of SAXS data (Section 4.3). Different parameterized structural minimal models of SVs (Section 4.4) are constructed considering information from cryo-EM on the shape of SVs, as well as cryo-EM data on the size distribution of SVs. Further, information from DLS on the size distribution of SVs and biochemical information on the molecular inventory of SVs are taken into account. The form factors corresponding to the minimal real-space models are derived and generalized (Section 4.5). Subsequently, these form factors are least-squares fitted to SAXS data from SVs (Section 4.6). All optimized isotropic models are falsified, while optimized anisotropic models are in excellent agreement with SAXS data, cryo-EM data and biochemical data. Finally, conclusions from this chapter are summarized (Section 4.7).

\subsection{Kinematic Approximation}

The scattering cross section for a dilute, polydisperse system of spherical core-shell particles of radius $R$ with the number size distribution $p(R)$, the volumes $V(R)$, and the scattering form factor $P(q, R)$ is given by (49)

$$
\frac{d \sigma(q)}{d \Omega}=\Delta \rho^{2} \int_{0}^{\infty} p(R) V(R)^{2} P(q, R) d R
$$


$V(R)$ is the dry volume of the particle defined as the total volume $V_{\text {tot }}$ minus the volume of the solvent core $V_{\text {core }} . \Delta \rho=M / V$ denotes the difference between the scattering length density of the solvent and the average scattering length density of the decorated bilayer shell. $M$ will be used below as the total excess scattering length of a particle.

The total number of electrons within the particle population is

$$
N_{e}=\left(\rho_{0}+\Delta \rho_{e}\right) \int_{0}^{\infty} p(R) V(R) d R
$$

with $\rho_{0}$ denoting the electron density of the solvent, and $\Delta \rho_{e}$ the average excess electron density of the decorated bilayer shell. The dry mass $m$ of the particle population can be obtained from the modified Lowry protein assay (see section 2.3), and can be directly linked to $N_{e}$, assuming a fixed ratio of 1.87 neutrons or protons per electron within the particles $(50,51)$ and considering the electron density of the buffer (333 electrons $/ \mathrm{nm}^{3}$ ). Thus, $\Delta \rho$ and the number size distribution $p(R)$ can both be obtained on an absolute scale. As always in scattering experiments, two solutions generally exist for $\Delta \rho$ (and thus $p(R))$ due to Babinet's principle. Here we choose $\Delta \rho>0$, in agreement with the existing data on bilayer densities (15).

\subsection{Model Independent Data Analysis}

Model independent data analysis approaches allow to deduce 3D structural information directly from SAXS data by employing numerical methods. In general, these approaches also require some a priori assumptions about the sample; model building of specific parametrized models is, however, not required.

\subsubsection{Indirect Fourier Transformation Method and Convolution Square Root Operation}

The Indirect Fourier Transformation (IFT) method (52) applied to the SAXS data of uncorrelated monodisperse particles of radius $R$ with $0 \leq R \leq R_{\max }$ reveals the pair distance distribution function $p(r)$, or equivalently, the correlation function $\gamma(r)$ within particles of finite size. The distance distribution function $p(r)$ and the correlation function $\gamma(r)$ are connected via the relation $p(r)=r^{2} \gamma(r)$. Further, the IFT can be generalized to account for interactions of particles (structure factors), for polydisperse systems of particles of given shape (53), and non-spherical particles (accounted by a shape factor) (54).

The basic idea of the IFT method is to follow the experiment from real space (sample) to experimental (data) space, and than to come back to real space by performing a Fourier transform (30). First, an optimized function system (linear combination of cubic B-splines $(55,56)$, for example) is established by assigning a value to $R_{\max }$ and by using the relations $0 \leq R \leq$ 
$R_{\text {max }}$. Second, the experimental data is approximated (smoothed) using the optimized function system by employing a weighted least-squares technique. If necessary, corrections for instrumental broadening (desmearing) are performed. Third, the smoothed data is transformed into real space. Care has to be taken to minimize termination effects occurring during transformation.

In case of several centro symmetric particle structures, the scattering density profile can be obtained by numerical deconvolution of the pair distance distribution function $(57,58,59)$ by employing the convolution square root operation. However, lack of centro symmetry of the particle structure effectively prohibits to deconvolute the pair distance distribution function.

\subsubsection{Ab-Initio Shape Analysis}

Pseudo-atomistic ab-initio modeling reveals the three dimensional shape and overall low resolution structure of native proteins in solution by employing multipole expansions (60). This technique only works for particles with a nearly homogeneous distribution of scattering length density, and is restricted to monodisperse particles, or in a modified version, to two-phase particles (61). In recent years it has been demonstrated that rigid body modeling against SAXS data can elucidate the overall structure and conformational changes of functional complexes, flexible macromolecules and assembly processes $(62,63)$.

However, this approach seems unsuited for large heterogeneous and complex structures like SVs. The number of free parameters of such pseudo-atomistic models with sufficient resolution to resolve details of the bilayer structure and the membrane proteins would be too high when compared to the number of data points obtainable by SAXS experiments.

\subsection{Direct Modeling}

Direct modeling aims at building form factor models of an analytical form, consistent with all previous knowledge about the sample, which can be least-squares fitted to the scattering data (64). Models are falsified, i.e. rejected, or found to be consistent with the scattering data. In the latter case, the optimized model parameters obtained are subsequently subject to structural interpretation, taking into account independent data from different quantitative experimental techniques like cryo EM, DLS and biochemical analysis $(15,17)$.

\subsection{Model Building: Integrated Approach}

The SV models are constructed on the basis of information from cryo electron micrographs, revealing their spherical core-shell structure of a protein decorated lipid bilayer, as well as data and information on the size distribution $p(R)$ of SVs obtained by cryo-EM and DLS. Biochemical data on the molecular inventory of the SV provides further constraints. 
In all SV models, a bimodal size distribution function $p(R)=p_{E M}(R)+p_{G}(R)$ was employed as shown in Fig. $4.4(B)$. The branch $p_{E M}(R)$ of $p(R)$ around approximately $R=20 \mathrm{~nm}$ has been determined by cryo-EM (smoothed) (15). The diameter of the SV was measured from bilayer surface to bilayer surface. The branch $p_{G}(R)$ of $p(R)$ around approximately $R=200 \mathrm{~nm}$ was calculated as a freely varied Gaussian contribution for $\mu-3 \sigma \leq R \leq \mu+3 \sigma$ given by

$$
p_{G}(R)=\frac{a}{\sqrt{2 \pi \sigma^{2}}} \exp \left(-\frac{(R-\mu)^{2}}{2 \sigma^{2}}\right),
$$

with mean $\mu$, standard deviation $\sigma$ and scaling parameter $a$ to account for the trace number of larger membranous particles in the sample visible by cryo-EM, see Fig. 2.2. Further details on the larger particles as obtained by cryo-EM are given in section 2.5 and (14). The branch $p_{G}(R)$ of $p(R)$ also assures consistency of the model with DLS data on the size distribution of the SV samples, see section 2.6. In the DOPS vesicle models, $p(R)$ was a freely varied Gaussian only.

The form factor models derived here are built from a central bilayer profile $(65,66,67)$ with added contributions on the inside (lumen) and outside of the SV model, accounting for the numerous proteins associated with the SV membrane. The spherically symmetric electron density profile of the bilayer is modeled by three concentric Gaussians (68), representing the head groups of the two lipid leaflets and the hydrophobic core. Note that protein residues associated with the head groups and trans-membrane protein segments are included in this contribution. The larger proteins, or protein clusters, of the inner and outer protein shells, which can be clearly seen in cryo-EM images $(14,15)$ are modeled as follows: (i) The proteins are not explicitly integrated into the model. Also an asymmetric bilayer profile has been tested in this case. (ii) The proteins are modeled by coronas of concentric Gaussians, or (iii) coronas of hard spheres, or (iv) coronas of Gaussian chains (69) attached to the inner and outer sides of the bilayer, respectively $(15,70,71,72,73)$.

A sketch of the corresponding model with attached Gaussian chains (iv) in real space is given in Fig. 4.1 (B). This approach is a generalization of a model originally derived for polymer modified micelles and liposomes (70,73). For comparison, Fig. 4.1 (A) shows a section through a molecular model of an average SV, based on space-filling models of macromolecules at near atomic resolution (reproduction from (14)).

The contribution of the hard spheres coronas, or Gaussian chains coronas, explicitly introduces an in-plane structure to the models, breaking the spherical symmetry. The individual hard spheres or Gaussian chains are assumed to be perfectly uncorrelated, forming an ideal gas on the sphere. The extension of the additional concentric Gaussians, the hard spheres and the Gaussian chains in the radial direction might be interpreted as the thickness of the protein layers, whereas the lateral extension (parallel to the membrane tangent plane) of the hard spheres or Gaussian chains may reflect the in-plane size of individual proteins, protein 

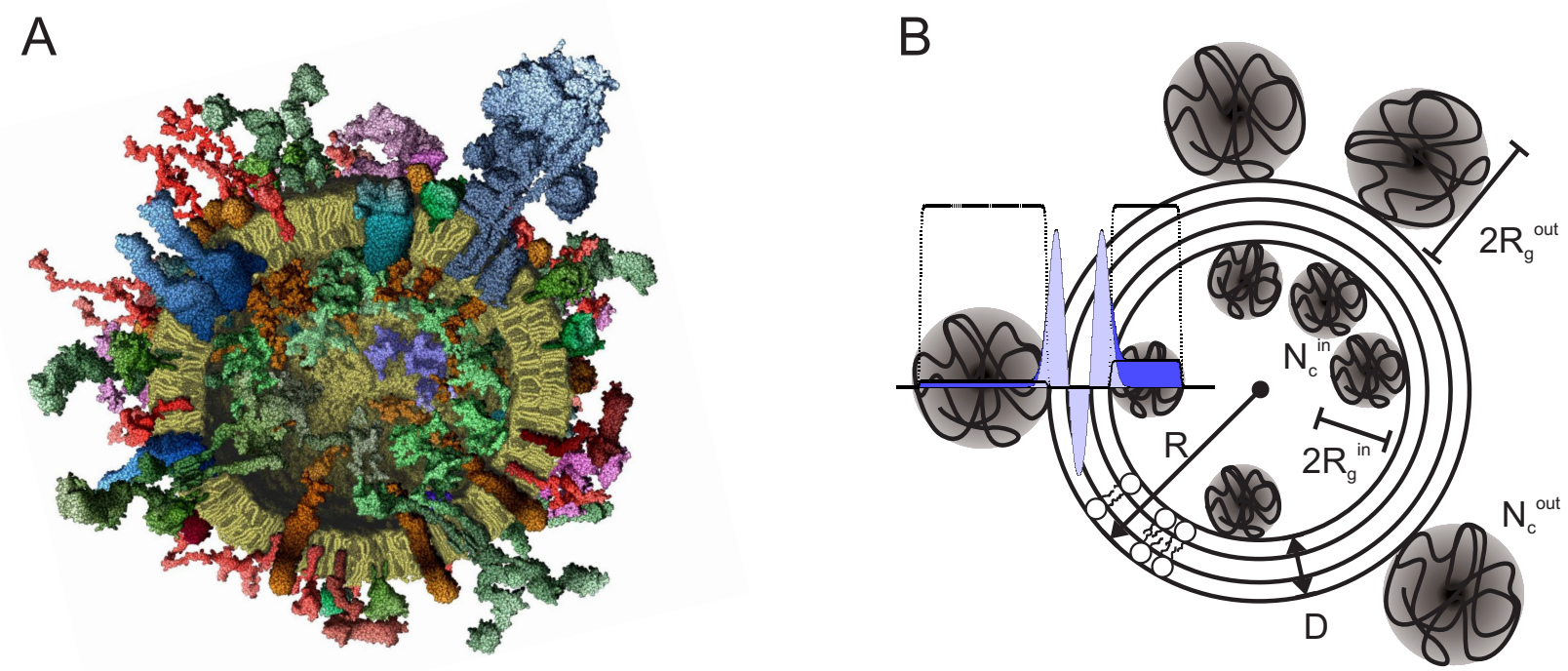

Figure 4.1: (A) Section through a molecular model of an average SV isolated from rat brain, based on space-filling models of macromolecules at near atomic resolution. Reproduction from (14). (B) Sketch of a real space model corresponding to an optimized scattering form factor consistent with the measured SAXS data. For details on the model and parameters see Tables 4.3 and 4.4, Fig. 4.4 and section 4.5. Reproduction from (15).

clusters and/or distinct lipid micro domains in a coarse grained sense. For the calculation of polydisperse populations the local structure of the concentric bilayer profile and, depending on the model, the additional terms describing the protein coronas were kept constant for all population members.

In addition to the parameters of the different models given in Tables 4.1, 4.2, and 4.5 three parameters reflecting the freely varied Gaussian component $p_{G}(R)$ of $p(R)$, accounting for larger membranous particles in the SV samples, and a small constant background, were subject to optimization during the fitting procedure of the form factor models to $I(q)$. The part $p_{E M}(R)$ of $p(R)$ representing the size distribution of the SVs as obtained by cryo-EM $(14,15)$ was kept constant for all fits. For both branches of $p(R)$, the same form factor was used. In particular the bilayer profile parameters, the parameters of the additional concentric Gaussians, or the density of hard spheres or Gaussian chains per surface area $\left(N_{c}^{i n} /\left(4 \pi\left(R-D-R_{g}^{i n}\right)^{2}\right)\right.$ and $\left.N_{c}^{\text {out }} /\left(4 \pi\left(R+R_{g}^{\text {out }}\right)^{2}\right)\right)$ were kept constant. The effective number of free parameters of the different models are 7 (3 Gaussians, symmetric profile), 9 (3 Gaussians, asymmetric profile), 11 (3 Gaussians forming symmetric profile, with 2 additional concentric Gaussians), and 12 (3 Gaussians forming symmetric profile, with hard spheres or Gaussian chains coronas attached). However, it should be emphasized that in practice part of the parameters were only free to vary within relatively narrow intervals due to the structural constraints to these parameters 
imposed by the model. Details on the different form factors corresponding to the real space models presented above, are given in section 4.5 .

\subsection{Derivation of Form Factors}

\subsubsection{Isotropic Models}

Equation 4.7 (below) gives the final form factor of the isotropic models used in the fitting procedures. The models are built from a central symmetric or asymmetric bilayer profile, with added spherically symmetric protein shells on the inside (lumen) and outside of the SV. The bilayer electron density profile is modeled by three concentric Gaussians (68), representing the headgroups of the two lipid leaflets and the hydrophobic core. Note that the amino acid residues associated with the headgroups and trans-membrane protein segments are included in this contribution. The inner and outer protein shells are modeled by concentric Gaussians attached to the inner and outer sides of the bilayer, respectively. The excess scattering length density of the bilayer profile is given by

$$
\rho(r)=\sum_{i} \rho_{i} \exp \left(-\frac{\left(r-R_{i}\right)^{2}}{2 t_{i}^{2}}\right),
$$

with the peak position $R_{i}$, amplitude $\rho_{i}$ and width $t_{i}$ with $i \in i n$, out, tail, inner, outer, for each of the three Gaussians representing the headgroups of the two leaflets and the tail region, and the inner and outer protein shell, respectively. The (characteristic) radius $R$ is defined as $R=R_{\text {out }}+t_{\text {out }} \sqrt{2 \pi} / 2$ mimicking a SV with the outer lipid bilayer surface at $r \simeq R$. To reduce the number of model parameters, we choose $R_{\text {tail }}=R-\left(t_{\text {out }}+t_{\text {tail }} / 2\right) \sqrt{2 \pi}, R_{\text {in }}=$ $R-\left(t_{\text {out }}+t_{\text {tail }}+t_{\text {in }} / 2\right) \sqrt{2 \pi}$. Thus, the total thickness $D$ of the bilayer is characterized by $D=\sqrt{2 \pi}\left(t_{\text {in }}+t_{\text {tail }}+t_{\text {out }}\right)$. Further, we choose $R_{\text {inner }}=R-\left(t_{\text {out }}+t_{\text {tail }}+t_{\text {in }}+t_{\text {inner }} / 2\right) \sqrt{2 \pi}$ and $R_{\text {outer }}=R+\left(t_{\text {outer }} / 2\right) \sqrt{2 \pi}$ so that the overall thickness of the synaptic membrane is characterized by the total thickness $D$ of the bilayer structure and the thickness of the inner and outer protein layers. The total excess scattering length with respect to the aqueous buffer is $\beta_{b}$. Depending on the specific model, we choose (i) $t_{\text {in }}=t_{\text {out }}$ (symmetric bilayer profile), (ii) $t_{\text {in }} \neq t_{\text {out }}$ (asymmetric bilayer profile), or (iii) $\rho_{\text {inner }}=\rho_{\text {outer }}=0$ (no protein shells). Note that the Gaussians representing the bilayer profile and the protein shells interpenetrate to some extent due to their tails.

The form factor $P(q, R)$ (see Eq. 4.1) of spherical particles can be written as

$$
P(q, R)=(F(q, R))^{2}
$$

where $F(q, R)$ is the normalized form factor amplitude, which can be calculated in kinematic scattering theory by the spherical Fourier Transform of the excess scattering length density 
$\rho(r)$, given by

$$
F(q, R)=\frac{4 \pi}{M} \int_{0}^{\infty} \rho(r) r^{2} \frac{\sin (q r)}{q r} d r
$$

with $M$ the excess scattering length of the particle. Thus, the form factor amplitude $F(q, R)$ corresponding to the above real space model can be calculated in kinematic scattering theory by inserting Eq. 4.4 into Eq. 4.6. The resulting one-dimensional Fourier integral can be readily calculated by changing the lower boundary of the integral from 0 to $-\infty$. This approximation is valid if the values of the peak positions $R_{i}$ are large when compared to the corresponding values of the thickness parameters $t_{i}$ of the concentric Gaussians (68). Subsequently, the form factor $P(q, R)$ corresponding to $F(q, R)$ is obtained by inserting $F(q, R)$ into Eq. 4.5, yielding the following result

$$
P(q, R)=\frac{1}{M^{2}} \beta_{b}^{2} F_{b}^{2}(q, R) .
$$

The symbols and functions are given below: $M=\beta_{b}$ denotes the excess scattering length of the bilayer profile. The normalized amplitude of the self-correlation term of the bilayer profile and the isotropic protein shells is given by

$$
F_{b}(q, R)=\frac{1}{\beta_{b}} \sum_{i} \beta_{b i} F_{b i}\left(q, R_{i}\right),
$$

with $i \in$ in, out, tail, inner, outer, and (68)

$$
F_{b i}\left(q, R_{i}\right)=\frac{1}{\beta_{b i}} 4 \sqrt{2} \pi^{3 / 2} t_{i} \rho_{i} \exp \left(-\frac{t_{i}^{2} q^{2}}{2}\right) \frac{1}{q}\left[t_{i}^{2} q \cos \left(q R_{i}\right)+R_{i} \sin \left(q R_{i}\right)\right],
$$

where $\beta_{b}{ }_{i}=\rho_{i} \frac{4 \pi}{3}\left(\left(R_{i}+t_{i} \sqrt{2 \pi} / 2\right)^{3}-\left(R_{i}-t_{i} \sqrt{2 \pi} / 2\right)^{3}\right)$ denotes the excess scattering mass of one peak of the profile (68).

\subsubsection{Anisotropic Models}

\section{Synaptic Vesicle Models}

Equation 4.34 (below) gives the final form factor for the anisotropic SV model with Gaussian chains coronas used in the fitting procedure. The model is built from a central bilayer profile with added protein coronas on the inside (lumen) and outside of the SV model.

The bilayer electron density profile is modeled by three concentric Gaussians (68), representing the headgroups of the two lipid leaflets and the hydrophobic core. Note amino acid residues associated with the headgroups and trans-membrane protein segments are included in this contribution. The inner and outer protein shells are modeled by Gaussian chains (69) attached to the inner and outer sides of the bilayer, respectively $(70,71,72,73)$. 
The Gaussian chain is a popular simple model for the description of global properties of polymers. However, the Gaussian chain does not describe correctly the local structure of a polymer (74). The conformation of the Gaussian chain is represented by the set of $(\mathrm{N}+1)$ position vectors $\left\{\mathbf{R}_{n}\right\}$ of the joints of the chain, or by the set of bond vectors $\left\{\mathbf{r}_{n}\right\}$, with $\mathbf{r}_{n}=\mathbf{R}_{n}-\mathbf{R}_{n-1}, n=1,2, . ., N(74)$. The bond vectors $\mathbf{r}_{n}$ of the Gaussian chain are randomly orientated, and the bond length has a Gaussian distribution given by (74)

$$
\psi(\mathbf{r})=\left(\frac{3}{2 \pi b^{2}}\right)^{3 / 2} \exp \left(-\frac{3 \mathbf{r}^{2}}{2 b^{2}}\right),
$$

so that $\left\langle\mathbf{r}^{2}\right\rangle=b^{2}$ with the effective bond length $b$. The conformational distribution function of such a Gaussian chain is given by (74)

$$
\Psi(\{\mathbf{r}\})=\prod_{n=1}^{N}\left(\frac{3}{2 \pi b^{2}}\right)^{3 / 2} \exp \left(-\frac{3 \mathbf{r}_{n}^{2}}{2 b^{2}}\right) .
$$

The scattering form factor of a Gaussian chain can be calculated (74). However, the scattering form factor amplitude of a Gaussian chain remains elusive, and an effective form factor amplitude has been derived and suggested for substitution $(70,75)$, see below.

The excess scattering length density of the bilayer profile is given by Eq. 4.4 with $i \in$ in, out, tail, for each of the three Gaussians representing the headgroups of the two leaflets and the tail region, respectively. The (characteristic) radius $R, R_{\text {tail }}, R_{i n}, D$ and $\beta_{b}$ are defined as for the the isotropic form factor models. To reduce the number of model parameters further, we choose $t_{\text {in }}=t_{\text {out }}$ (symmetric bilayer profile). There are $N_{c}^{\text {in }}$ and $N_{c}^{\text {out }}$ Gaussian chains distributed randomly and without correlations forming the inner and outer protein shell, respectively. The individual Gaussian chains are characterized by their root-mean-square radius of gyration, $R_{g}^{\text {in }}$ and $R_{g}^{\text {out }}$, given by (74)

$$
R_{g}^{i}=\sqrt{\frac{1}{N} \sum_{n=1}^{N}\left\langle\left(\mathbf{R}_{n}^{i}-\mathbf{R}_{C M}^{i}\right)^{2}\right\rangle}
$$

with $i=$ in, out, the set of position vectors $\left\{\mathbf{R}_{n}^{i}\right\}$ of the joints of the Gaussian chain, and the position vector $\mathbf{R}_{C M}^{i}$ of the centre of mass of the corresponding Gaussian chain, defined by (74)

$$
\mathbf{R}_{C M}^{i}=\frac{1}{N} \sum_{n=1}^{N} \mathbf{R}_{n}^{i} .
$$

The Gaussian chains representing the inner and outer protein shell have the common average excess scattering length density $\rho_{c}$. The distance between the inner headgroup maximum of the bilayer profile and the center of mass of the Gaussian chains facing the lumen is taken to be $t_{i n} \sqrt{2 \pi} / 2+R_{g}^{i n}$, and the distance between the outer headgroup maximum and the center of mass of the Gaussian chains facing outwards is taken to be $t_{\text {out }} \sqrt{2 \pi} / 2+R_{g}^{\text {out }}$. This limits the 
penetration of the Gaussian chains into the bilayer, although there is some remaining overlap, mostly due to the extending tails of the bilayer profile (70).

\section{Anisotropic Form Factors: General Considerations}

In general, the normalized form factor amplitude $F(\mathbf{q}, \mathbf{r})$ corresponding to a model of an arbitrarily shaped particle is given within the kinematic approximation by

$$
F(\mathbf{q}, \mathbf{r})=\frac{1}{M} \int_{V} \rho(\mathbf{r}) \exp (-i \mathbf{q} \cdot \mathbf{r}) d \mathbf{r}
$$

with $V$ the volume of the particle and $\rho(\mathbf{r})$ the excess scattering length density of the particle, relative to the scattering length density of the solvent. Further, $M$ denotes the excess scattering length of the particle.

The form factor amplitude $F(\mathbf{q}, \mathbf{r})$ can be calculated by considering the form factor amplitudes $F_{i}(\mathbf{q}, \mathbf{r})$ corresponding to the different parts of a decomposition of the particle into $n$ parts with

$$
F(\mathbf{q}, \mathbf{r})=\frac{1}{M} \sum_{i=1}^{n} M_{i} F_{i}(\mathbf{q}, \mathbf{r})
$$

where the $F_{i}(\mathbf{q}, \mathbf{r})$ are defined in analogy to Eq. 4.14 by

$$
F_{i}(\mathbf{q}, \mathbf{r})=\frac{1}{M_{i}} \int_{V_{i}} \rho_{i}(\mathbf{r}) \exp (-i \mathbf{q} \cdot \mathbf{r}) d \mathbf{r}
$$

with $V_{i}$ the volume of part $i$ of the particle, $\rho_{i}(\mathbf{r})$ the excess scattering length density of part $i$ of the particle, relative to the scattering length density of the solvent, and $M_{i}$ the excess scattering mass of part $i$ of the particle.

The normalized scattering form factor $P(\mathbf{q}, \mathbf{r})$ of the particle is given by

$$
P(\mathbf{q}, \mathbf{r})=|F(\mathbf{q}, \mathbf{r})|^{2}
$$

and is related to the scattering cross section of the particle by

$$
\frac{d \sigma(\mathbf{q})}{d \Omega}=\Delta \rho^{2} V^{2} P(\mathbf{q}, \mathbf{r})
$$

with $\Delta \rho$ the average excess scattering length density of the particle, and $V$ the volume of the particle.

Further, the scattering cross section of a particle averaged over all orientations is given by

$$
\frac{d \sigma(q)}{d \Omega}=\Delta \rho^{2} V^{2}<P(\mathbf{q}, \mathbf{r})>
$$

where $\langle. .>$ denotes the integration(s) for the calculation of the orientational averaging, and $q$ the modulus of the scattering vector $\mathbf{q}$. The orientational averaged scattering form factor can be written with Eq. 4.15 , Eq. 4.17 and $\left|\sum_{i=1}^{n} F_{i}(\mathbf{q}, \mathbf{r})\right|^{2}=\sum_{i=1}^{n} \sum_{j=1}^{n} F_{i}(\mathbf{q}, \mathbf{r}) F_{j}^{*}(\mathbf{q}, \mathbf{r})$ as 


$$
<P(\mathbf{q}, \mathbf{r})>=<\sum_{i=1}^{n} \sum_{j=1}^{n} F_{i}(\mathbf{q}, \mathbf{r}) F_{j}^{*}(\mathbf{q}, \mathbf{r})>.
$$

Considering the linearity of the integrals representing the orientational averaging, Eq. 4.20 can be written as

$$
<P(\mathbf{q}, \mathbf{r})>=\sum_{i=1}^{n} \sum_{j=1}^{n}<F_{i}(\mathbf{q}, \mathbf{r}) F_{j}^{*}(\mathbf{q}, \mathbf{r})>.
$$

Thus, the entire scattering form factor may be averaged over all orientations (Eq. 4.20), or alternatively, the different auto-correlation and cross-correlation terms of the scattering form factor may be separately averaged over all orientations (Eq. 4.21).

The orientationally averaged form factor $\langle P(\mathbf{q}, \mathbf{r})\rangle=P(q, R)$ corresponding to a model structure composed of $N$ isotropic particles, with scattering amplitudes $F_{i}\left(q, R_{i}\right)$ and excess scattering masses $M_{i}$ is given by (76)

$$
P(q, R)=\frac{1}{M^{2}} \sum_{i, j=1}^{N} M_{i} M_{j} F_{i}\left(q, R_{i}\right) F_{j}\left(q, R_{j}\right) \frac{\sin \left(q r_{i j}\right)}{q r_{i j}}
$$

with $r_{i j}$ the distance between the centers of the $i$ th and $j$ th subunit, and

$$
M=\sum_{i=1}^{N} M_{i}
$$

Starting from Eq. 4.22, the form factor can be calculated corresponding to a particle composed of an isotropic core structure with $p$ concentric spherical coronas, each consisting of $N_{i}$ uncorrelated, isotropic structures. For simplicity, all of these isotropic 'blobs' within one corona are assumed here to have the same structural properties. The derivation of the form factor follows $(70,71,72)$. In particular, the interference terms of scattering from 'blobs' belonging to one corona are calculated by averaging the phase factor $\sin (q r) /(q r)$ over the proper weighting of the distance $r(72,77)$. For simplicity, we assume that the 'blobs' of the coronas form an ideal gas on spherical shells $(70,71,72)$. Thus, the proper weighting functions of the distance $r$ are the pair distance distribution functions $p_{i}(r)$ of infinitely thin shells of radius $R_{i}(70,71,72)$, given by $(78,79)$

$$
p_{i}(r)=\frac{2 r}{\left(2 R_{i}\right)^{2}}
$$

for $0 \leq r<2 R_{i}$, and

$$
p_{i}(r)=\frac{1}{2 R_{i}}
$$

for $r=2 R_{i}$. The interference terms of scattering contributions originating from different coronas are calculated as the product of the square roots of the interference terms originating from 'blobs' within each of the corresponding coronas $(15,73)$. The final form factor, a generalization 
of the expressions given in $(15,70,71,72,73)$, is given by

$$
\begin{aligned}
P(q, R)=\frac{1}{M^{2}} \times\left[\begin{array}{l} 
\\
\end{array}\right. & M_{c}^{2} F_{c}^{2}(q, R) \\
& +\sum_{i=1}^{p} N_{i} M_{i}^{2} F_{i}^{2}\left(q, r_{i}\right) \\
& +\sum_{i=1}^{p} 2 N_{i} M_{i} M_{c} F_{c}(q, R) F_{i}\left(q, r_{i}\right) \frac{\sin \left(q R_{i}\right)}{q R_{i}} \\
& +\sum_{i, j=1}^{p}\left(N_{i} N_{j} M_{i} M_{j} F_{i}\left(q, r_{i}\right) F_{j}\left(q, r_{j}\right) \frac{\sin \left(q R_{i}\right)}{q R_{i}} \frac{\sin \left(q R_{j}\right)}{q R_{j}}\right. \\
& \left.\left.\quad-\delta_{i j} N_{i} M_{i}^{2} F_{i}^{2}\left(q, r_{i}\right)\left(\frac{\sin \left(q R_{i}\right)}{q R_{i}}\right)^{2}\right)\right],
\end{aligned}
$$

where $M$ denotes the total excess scattering mass, given by

$$
M=M_{c}+\sum_{i=1}^{p} N_{i} M_{i}
$$

with $M_{c}$ the excess scattering mass of the core, $N_{i}$ the number of isotropic 'blobs' with excess scattering mass $M_{i}$ forming the corona at radius $R_{i}$. Further, $F_{c}(q, R)$ and $F_{i}\left(q, r_{i}\right)$ denote the scattering amplitude of the core, and the 'blobs', respectively. Finally, $\delta_{i j}$ the Kronecker Delta, with $\delta_{i j}=1$ for $i=j$ and $\delta_{i j}=0$ for $i \neq j$. Details on the different terms in Eq. 4.26 are given below. The terms in the first two lines represent the weighted contributions from the self-interference of the scattering from the core and the 'blobs' of the coronas. The terms in the third line represent the weighted interference of the scattering from the core and the 'blob' coronas. Further, the terms in the last two lines represent the weighted self-interference $(i=j)$ and interference $(i \neq j)$ of scattering from the 'blob' coronas. The terms in the last line assure proper weighting of the self-interference terms $(70,71,72)$. Note that the form factor given by Eq. 4.26 corresponds to an explicitly anisotropic model structure since the contributions from the isotropic 'blobs' forming the coronas break the spherical symmetry of the model $(70,71,72)$. The form factor given by Eq. 4.26 can be further generalized to correspond to particles with, for example, ellipsoidal or cylindrical cores and coronas, following the approach taken in $(70,71)$.

The models above assume that the coronal 'blob' structures are uncorrelated, forming an ideal gas on a surface. The effects due to the ideal gas assumption for the 'blobs' were investigated for form factor models corresponding to a population of uncorrelated particles each consisting of two 'blob' point scatterers randomly distributed on a spherical surface of radius $R_{1}=1$. The normalized form factor corresponding to the above model within the ideal gas approximation for the 'blobs' is given by Eq. 4.26 and Eq. 4.27 with $p=1$ and $M_{c}=0$ yielding the following result

$$
P\left(q, R_{1}\right)=\frac{1}{M^{2}}\left(N_{1} M_{1}^{2} F_{1}^{2}(q)+\left(N_{1}-1\right) N_{1} M_{1}^{2} F_{1}^{2}(q)\left(\frac{\sin \left(q R_{1}\right)}{q R_{1}}\right)^{2}\right),
$$


with $M_{1}=1$ the excess scattering mass of one 'blob', $N_{1}=2$ the number of 'blobs', $F_{1}(q)=1$ the form factor amplitude of a point 'blob', and $M$ the total excess scattering mass given by Eq.4.27. A form factor $P^{\prime}\left(q, R_{1}\right)$ which takes into account the correlations between two isotropic 'blob' scatterers on a spherical surface was calculated by replacing the pair distance distribution function $p_{i}(r)$ of a homogeneous spherical shell in Eq. 4.24 and Eq. 4.25 by the pair distance distribution function of two points on a spherical surface, given by

$$
p_{i}(r)=\frac{3}{\left(2 R_{i}\right)^{2}} \sqrt{r^{2}-\left(\frac{r^{2}}{2 R_{i}}\right)^{2}}
$$

with $i=1$ and $R_{1}=1$. As above, the interference term of the 'blobs' was calculated by averaging the phase factor $\sin (q r) /(q r)$ over the weighting of the distance $r$ given by Eq. 4.29, yielding for the entire form factor the following result

$$
P^{\prime}\left(q, R_{1}\right)=\frac{1}{M^{2}}\left(N_{1} M_{1}^{2} F_{1}^{2}(q)+\left(N_{1}-1\right) N_{1} M_{1}^{2} F_{1}^{2}(q) \frac{3 \pi \text { Struve } H\left[1,2 q R_{1}\right]}{8 q^{2} R_{1}^{2}}\right),
$$

with $M_{1}=1$ the excess scattering mass of one 'blob', $N_{1}=2$ the number of 'blobs', $M=N_{1} M_{1}$ the total excess scattering mass, $F_{1}(q)=1$ the form factor amplitude of a 'blob', $R_{1}=1$ the radius of the spherical surface, and Struve $H[n, z]$ the Struve function given by (80)

$$
\text { Struve } H[n, z] \equiv \frac{2\left(\frac{1}{2} z\right)^{n}}{\Gamma\left(n+\frac{1}{2}\right) \Gamma\left(\frac{1}{2}\right)} \int_{0}^{\infty}\left(1-t^{2}\right)^{n-\frac{1}{2}} \sin (z t) d t
$$

with $\Gamma(z)$ the Gamma function, given in Euler's integral form by (81)

$$
\Gamma(z) \equiv \int_{0}^{\infty} t^{z-1} \exp (-t) d t
$$

The relative deviation $D(q)$ in \% of the model form factor employing the ideal gas approximation (Eq. 4.28) from the model form factor taking into account the correlations between the two 'blob' scatterers (Eq. 4.30) is given by

$$
D(q)=\frac{P^{\prime}\left(q, R_{1}\right)-P\left(q, R_{1}\right)}{P^{\prime}\left(q, R_{1}\right)} \times 100
$$

and was evaluated numerically using MATLAB, version 7.5.0.342 (R2007b), The MathWorks Inc.

Figure 4.2 (lower part) shows a comparison of the graphs obtained by plotting the form factors given by Eq. 4.28 (solid blue line) and Eq. 4.30 (dashed green line) with $R_{1}=1$ for $0.1 \leq q \leq 10$ inverse unit lengths. Figure 4.2 (upper part) shows the absolute value of the relative deviation $D(q)$ in $\%$ of the form factor values (solid red line). The absolute value of $D(q)$ is found to be smaller than $9 \%$, with the global maximum around $q \approx 2.2$ inverse length units. We speculate that the relative deviation of the model with the ideal gas approximation from the model taking into account the correlations between the 'blob' structures decreases 


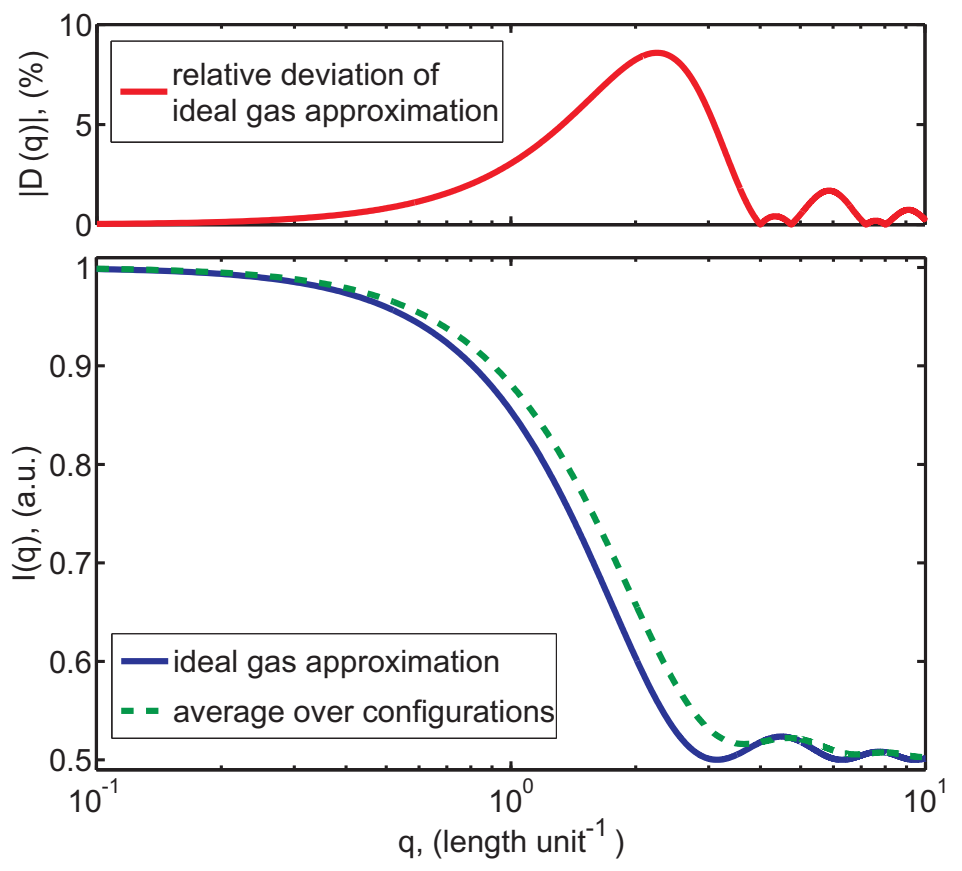

Figure 4.2: Calculated scattering curves of two point scatterers on a spherical shall of radius $R=1$. Model form factor within ideal gas approximation (solid blue line), model form factor taking into account correlations between the point scatteres on the spherical surface (dashed green line), and absolute value of the relative deviation $D(q)$ in \% of the model employing the ideal gas approximation (solid red line).

monotonically for larger $N_{1}$ with $N_{1}>2$. Thus, the ideal gas approximation has relatively small effects on the corresponding model form factor $P(q, R)$ for $N_{1} \geq 2$ and seems to be a reasonable assumption in many cases. However, especially for small $N_{1}$, e.g. $N_{1}=2$, it might be possible to falsify models corresponding to Eq. 4.28 by experimental data from suited systems, while the model corresponding to Eq. 4.30 might describe such data well.

In summary, scattering form factors may be calculated corresponding to complex, anisotropic models without the need to perform the orientational average for the whole model form factor, but instead make use of already orientationally averaged, or isotropic components, or to calculate corresponding expressions for the individual correlation terms of the form factor. Such approaches may significantly facilitate the calculation, numerical implementation and optimization of more complex model form factors. Further, there is no need to explicitly perform orientational averaging for scattering form factors corresponding to models consisting of a concentric isotropic core structure with uncorrelated isotropic 'blob' structures forming an ideal gas on spheres concentric to the core structure, although such models are explicitly anisotropic. The ideal gas approximation for the 'blobs' has typically only relatively small effects on such model form factors and seems to be overall a reasonable assumption for the modeling of SVs here. 


\section{Anisotropic Form Factors: Synaptic Vesicle Models}

The form factor corresponding to the above anisotropic SV model with Gaussian chains coronas can be calculated in kinematic scattering theory by employing Eq. 4.26, yielding the following result

$$
\begin{aligned}
& P(q, R)=\frac{1}{M^{2}} \times\left[\quad \beta_{b}^{2} F_{b}^{2}(q, R)\right. \\
& +\sum_{i=\text { in }, \text { out }} N_{c}^{i} \beta_{c}^{i 2} P_{c}^{i}(q) \\
& +\sum_{i=\text { in }, \text { out }} 2 N_{c}^{i} \beta_{b} \beta_{c}^{i} S_{b c}^{i}(q, R) \\
& +\sum_{i=\text { in }, \text { out }} N_{c}^{i}\left(N_{c}^{i}-1\right) \beta_{c}^{i 2} S_{c}^{i}(q, R) \\
& \left.+\quad S_{c}^{\text {in out }}(q, R) 2 \prod_{i=\text { in }, \text { out }} N_{c}^{i} \beta_{c}^{i}\right] .
\end{aligned}
$$

The symbols and functions are given below: $M=\beta_{b}+N_{c}^{\text {in }} \beta_{c}^{\text {in }}+N_{c}^{\text {out }} \beta_{c}^{\text {out }}$ denotes the excess scattering length, with $\beta_{c}^{i}=\rho_{c} R_{g}^{i} 4 \pi / 3$ and $i=i n$, out representing the total excess scattering length of a single chain on the outside and on the inside of the bilayer profile, respectively. The normalized amplitude of the self-correlation term of the bilayer profile is given by Eq. 4.8 with Eq. 4.9 where $\beta_{b} i$ is defined as for the isotropic form factor models.

The self-correlation terms of the Gaussian chains are given by Debye functions (74)

$$
P_{c}^{i}(q)=\frac{2\left[\exp \left(-x^{i}\right)-1+x^{i}\right]}{x^{i}}
$$

with $x^{i}=q^{2} R_{g}^{i 2}$ and $i=i n$, out for the inner and outer chains, respectively. The interference cross-terms $S_{b c}^{\text {in }}(q, R)$ and $S_{b c}^{\text {out }}(q, R)$ between the bilayer and the Gaussian chains on the inside and outside, are given by

$$
S_{b c}^{i}(q, R)=F_{b}(q, R) \psi^{i}\left(x^{i}\right) \frac{\sin \left(q\left[R_{\text {tail }} \mp\left(D / 2+R_{g}^{i}\right)\right]\right)}{q\left[R_{\text {tail }} \mp\left(D / 2+R_{g}^{i}\right)\right]},
$$

with $i=$ in, out and $\psi^{i}\left(x^{i}\right)=\left[1-\exp \left(-x^{i}\right)\right] / x^{i}$ the effective form factor amplitude of the Gaussian chains (75).

The product of the scattering form factor amplitudes of the chains belonging to one of the chain layers with the scattering form factor amplitude of an infinite thin shell is equivalent to a convolution of the corresponding scattering density distributions in real space (71) and accounts for the fact that the Gaussian shells are located on a spherical shell.

$$
S_{c}^{i}(q, R)=\left(\psi^{i}\left(x^{i}\right) \frac{\sin \left(q\left[R_{\text {tail }} \mp\left(D / 2+R_{g}^{i}\right)\right]\right)}{q\left[R_{\text {tail }} \mp\left(D / 2+R_{g}^{i}\right)\right]}\right)^{2},
$$


with $i=i n$, out. The interference term between the chains of the inner and outer shells is taken into account by

$$
S_{c}^{\text {in out }}(q, R)=\prod_{i=\text { in out }} \psi^{i}\left(x^{i}\right) \frac{\sin \left(q\left[R_{\text {tail }} \mp\left(D / 2+R_{g}^{i}\right)\right]\right)}{q\left[R_{\text {tail }} \mp\left(D / 2+R_{g}^{i}\right)\right]} .
$$

The anisotropic form factor, where spherical particles were placed on the inside and outside of a symmetric bilayer profile instead of the Gaussian chains, is similar to that given in Eq. 4.34, except that $P_{c}^{i}$ in Eq. 4.35 and $\psi^{i}$ in Eq. 4.36 are replaced by the scattering form factor of spheres and the scattering form factor amplitude of spheres, respectively. The spheres are of radii $R^{\text {in }}$ and $R^{\text {out }}$, the number of free parameters is the same as for the model with the attached Gaussian chains.

The model form factors calculated in this section correspond to model particles with spherically symmetric core structures and (effectively) spherically symmetric structures (Gaussian chains, hard spheres) attached at certain radii which are uncorrelated in the plane of the surface of the sphere (ideal gas on a sphere). However, the approach chosen in Eqs. 4.7 and 4.34 to assemble the final form factor from the sum of the different auto-correlation and cross-correlation terms representing different parts of the model particle is not restricted to model systems with spherically symmetric cores, and can, for example, be generalized to models with ellipsoidal and cylindrical cores $(70,71)$.

\subsection{Model Falsification Against SAXS Data}

The different form factor models were least-squares fitted to SAXS data. Least-squares fitting was performed using the lsqnonlin routine of MATLAB Optimization Toolbox (Version 7.5.0.342 (R2007b), The MathWorks Inc.), dedicated to solve nonlinear least-squares problems. For details on the structure of the employed MATLAB code and the object function see Appendix.

The quality of a model fit $f(x)$ with $p$ free model parameters to $N$ data points of experimentally estimated photon counts $y_{i}$ with empirical variances $\sigma_{i}^{2}$ was assessed by the value of the reduced $\chi^{2}$ given by

$$
\text { reduced } \chi^{2}=\frac{\sum_{i=1}^{N} \frac{\left(y_{i}-f\left(x_{i}\right)\right)^{2}}{\sigma_{i}^{2}}}{N-p-1} .
$$

The variances $\sigma_{i}^{2}$ were calculated by propagating the counting errors from the photon counts of the individual detector pixels through the data reduction and correction process described in Section 3.1. Cross-correlations between pixels were neglected.

An optimized model is rejected if the value of the reduced $\chi^{2}$ for the best fit to the SAXS data is large when compared to 1 . Further, an optimized model is rejected if it suggests a structure which can be falsified by cryo-EM or biochemical data. 
Nonlinear regression inference is performed using the linear approximation to the expectation function. Approximate marginal confidence intervals (95\%) for the parameter values are calculated using the nlparci routine of MATLAB Statistics Toolbox (Version 7.5.0.342 (R2007b), The MathWorks Inc.). For the nonlinear models the Jacobi matrix is evaluated at the least squares parameter estimates as obtained from the lsqnonlin fitting routine (see above) $(82,83)$. Geometrically stated, the expectation surface is approximated by the tangent plane at the least squares parameter estimates, and the true parameter coordinate system is approximated by a linear coordinate system on the approximating tangent plane (82). Note that the extent to which the calculated approximate marginal confidence intervals adequately delineate the regions of reasonable parameter values is determined by the adequacy of the linear approximation to the expectation function (82). One main source of systematic errors is the uncertainty in the absolute scale of the data. 


\subsubsection{Native Synaptic Vesicles}

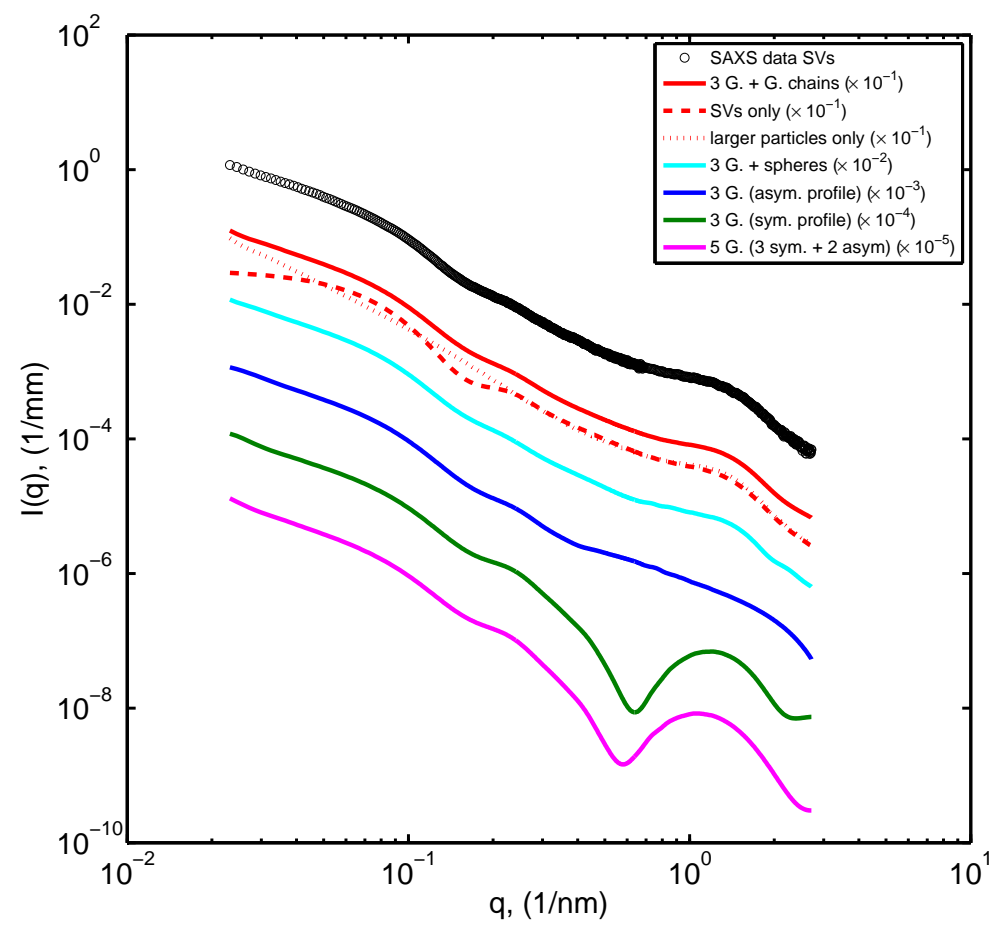

Figure 4.3: SV SAXS data (open circles, black). Fit of anisotropic model (three Gaussians forming symmetric bilayer profile, with Gaussian chains attached) to the SV data (full line, red), and contributions to the model calculation originating from the SVs (dashed line, red) and the larger membranous particles (dotted line, red). Corresponding electron density profile and size distribution are given in Fig. 4.4. Fit of anisotropic model (three Gaussians forming symmetric bilayer profile, with hard spheres attached) to the SV data (full line, cyan). Fit of isotropic model (three Gaussians forming asymmetric bilayer profile) to the SV data (full line, blue). Fit of isotropic model (three Gaussians forming symmetric bilayer profile) to the SV data (full line, dark green). Fit of isotropic model (three Gaussians forming symmetric bilayer profile, plus one additional Gaussian attached to each sides of the profile) to the SV data (full line, magenta). Curves partly shifted downwards for clarity, as detailed in the key. Figure adapted from (17).

Figure 4.3 shows data $I(q)$ vs $q$ from SVs in aqueous buffer (open circles, black), and leastsquares fits of isotropic and anisotropic form factor models to the data. The isotropic form factors consist of concentric Gaussians forming symmetric or asymmetric scattering density profiles. Three Gaussians forming a symmetric profile (solid dark green line , reduced $\chi^{2}=$ 602), five Gaussians of which three are forming a symmetric core profile (solid magenta line, reduced $\chi^{2}=578$ ), three Gaussians forming an asymmetric profile (solid blue line, reduced $\chi^{2}=$ 
34.1). A bimodal size polydispersity distribution function $p(R)$ was used, with one branch corresponding to cryo-EM data on the size distribution of the SVs, and a second branch corresponding to larger membranous particles, modeled by a freely varied Gaussian distribution.

Table 4.1: Parameter values of the decorated bilayer as obtained from the optimized isotropic SV SAXS model fits shown in Fig. 4.3. The models consist of concentric Gaussian distributed scattering densities. Parameter values of the corresponding optimized bimodal polydispersity distributions of the SVs are given in the text.

\begin{tabular}{|c|c|c|c|c|c|}
\hline Model & $\begin{array}{l}\rho_{c \text { in }}^{*} \\
t_{c \text { in }} \sqrt{2 \pi}\end{array}$ & $\begin{array}{l}\rho_{i n}^{*} \\
t_{i n} \sqrt{2 \pi}\end{array}$ & $\begin{array}{l}\rho_{\text {tail }}^{*} \\
t_{\text {tail }} \sqrt{2 \pi}\end{array}$ & $\begin{array}{l}\rho_{\text {out }}^{*} \\
t_{\text {out }} \sqrt{2 \pi}\end{array}$ & $\begin{array}{l}\rho_{c \text { out }}^{*} \\
t_{\text {c out }} \sqrt{2 \pi}\end{array}$ \\
\hline 3 Gaussians & - & 2.24 a.u. & -1 a.u. & 0.05 a.u. & - \\
\hline (asymmetric bilayer profile) & - & $1.14 \mathrm{~nm}$ & $0.97 \mathrm{~nm}$ & $8.88 \mathrm{~nm}$ & - \\
\hline 3 Gaussians & - & 2.29 a.u. & -1 a.u. & 2.29 a.u. & - \\
\hline (symmetric bilayer profile) & - & $1.64 \mathrm{~nm}$ & $2.38 \mathrm{~nm}$ & $1.64 \mathrm{~nm}$ & - \\
\hline 5 Gaussians & 0.06 a.u. & 1.43 a.u. & -1 a.u. & 1.43 a.u. & 0.40 a.u. \\
\hline (asymmetric bilayer profile)\# & $2.10 \mathrm{~nm}$ & $1.61 \mathrm{~nm}$ & $2.11 \mathrm{~nm}$ & $1.61 \mathrm{~nm}$ & $1.52 \mathrm{~nm}$ \\
\hline
\end{tabular}

* Normalized to $\rho_{\text {tail }}=-1$.

\# 3 Gaussians form symmetric core profile.

In addition to the parameters given in Tables 4.1 the following parameters have been obtained from the fits (model with three Gaussians (symmetric bilayer profile), model with five Gaussians, model with three Gaussians (asymmetric bilayer profile)): mean radius larger particles $(\mu=$ $160.7 \mathrm{~nm}, \mu=180.1 \mathrm{~nm}, \mu=127.0 \mathrm{~nm})$, width distribution larger particles $(\sigma=45.2 \mathrm{~nm}$, $\sigma=50.2 \mathrm{~nm}, \sigma=51.7 \mathrm{~nm})$, number larger particles $(1.55 \%, 1.61 \%, 2.47 \%)$ and a small constant background $\left(7 \times 10^{-5} \mathrm{~mm}^{-1}, 3 \times 10^{-5} \mathrm{~mm}^{-1},-3 \times 10^{-5} \mathrm{~mm}^{-1}\right)$. The excess scattering densities $\rho_{i}$ (relative to aqueous buffer) have been scaled to arbitrary units such that $\rho_{\text {tail }}=-1$. Parameters are explicitly defined in section 4.5.

Only the form factor consisting of three Gaussians forming an asymmetric profile is capable of describing the SV SAXS data, at least qualitatively. However, the parameter values of the profile do not correspond to a physically meaningful model. The maximum excess scattering density of the inner leaflet is about 45 times higher than for the outer leaflet. The model parameters would suggest the structure of a lipid monolayer with a thickness of about $3 \mathrm{~nm}$. However, EM shows a more or less roughly symmetrical bilayer structure of about $5 \mathrm{~nm}$ thickness. Further, the indirect Fourier transformation method (52) applied to the SAXS data, followed by the numerical deconvolution of the pair distance distribution function $(57,58,59)$ supports the view that a spherical symmetric model is not suited to describe the data (data not shown). 
Elliptically deformed models consisting of a bilayer profile, similar to the isotropic models above, have also been falsified (data not shown).

The anisotropic models consist of a symmetric core profile (three concentric Gaussians) with either attached hard spheres (solid cyan line, reduced $\chi^{2}=4.18$ ), or with attached Gaussian chains (solid red line, reduced $\chi^{2}=2.84$ ) on both sides of the bilayer profile. The dashed and dotted red lines depict the contributions of the two branches of the bimodal size distribution function $p(R)$ of the model consisting of three Gaussians with Gaussian chains coronas. Again a bimodal size polydispersity distribution function $p(R)$ was used, as defined above.

Table 4.2: Parameter values of the decorated bilayer as obtained from the optimized anisotropic SV SAXS model fits shown in Fig. 4.3. The models consist of three concentric Gaussians (symmetric bilayer profile), with coronas attached to both sides. Parameter values of the corresponding optimized bimodal polydispersity distributions of the SVs are given in the text.

\begin{tabular}{|c|c|c|c|c|c|}
\hline Model & $\begin{array}{l}\rho_{\text {in }, \text { out }}^{*} \\
\rho_{\text {tail }}^{*} \\
\end{array}$ & $\begin{array}{l}t_{\text {in,out }} \sqrt{2 \pi} \\
t_{\text {tail }} \sqrt{2 \pi}\end{array}$ & $\rho_{c}^{*}$ & $\begin{array}{l}R_{g}^{\text {in }} \\
R_{g}^{\text {out }} \\
\end{array}$ & $\begin{array}{l}N_{c}^{\text {in }} /\left(4 \pi\left(R-D-R_{g}^{\text {in }}\right)^{2}\right) \\
N_{c}^{\text {out }} /\left(4 \pi\left(R+R_{g}^{\text {out }}\right)^{2}\right) \\
\end{array}$ \\
\hline Hard spheres coronas\# & $\begin{array}{l}0.18 \text { a.u. } \\
-1 \text { a.u. }\end{array}$ & $\begin{array}{l}2.9 \mathrm{~nm} \\
0.6 \mathrm{~nm}\end{array}$ & 0.28 a.u. & $\begin{array}{l}2.4 \mathrm{~nm} \\
5.6 \mathrm{~nm}\end{array}$ & $\begin{array}{l}13.75 \times 10^{-3} \mathrm{~nm}^{-2} \\
0.42 \times 10^{-3} \mathrm{~nm}^{-2}\end{array}$ \\
\hline Gaussian chains col & $\begin{array}{l}1.63 \text { a.u. } \\
-1 \text { a.u. }\end{array}$ & $\begin{array}{l}1.8 \mathrm{~nm} \\
2.1 \mathrm{~nm}\end{array}$ & 1.81 a.u. & $\begin{array}{l}3.2 \mathrm{~nm} \\
5.7 \mathrm{~nm}\end{array}$ & $\begin{array}{l}7.09 \times 10^{-3} \mathrm{~nm}^{-2} \\
0.47 \times 10^{-3} \mathrm{~nm}^{-2}\end{array}$ \\
\hline
\end{tabular}

\footnotetext{
* Normalized to $\rho_{\text {tail }}=-1$.

\# $R_{g}^{i}=R^{i}, i=$ in, out.
}

In addition to the parameters given in Table 4.2 the following parameters have been obtained from the fits (model hard spheres coronas, model Gaussian chains coronas): mean radius larger particles $(\mu=232.5 \mathrm{~nm}, \mu=210.1 \mathrm{~nm})$, width distribution larger particles $(\sigma=54.5 \mathrm{~nm}$, $\sigma=50.2 \mathrm{~nm})$, number density of larger particles $(0.68 \%, 0.86 \%)$ and a small constant background. Details on the form factors are given in section4.5.

The parameter values as obtained from the optimized anisotropic SAXS models (as given in Table 4.2), with 95\% marginal confidence intervals and calibrated to an absolute scale, are given in Table 4.3 .

For illustration, some properties of the the best-fit SV model structure as obtained from the model with Gaussian chains attached for a SV of the most frequent size are given in Table 4.4.

Figure $4.4(A)$ shows a sketch of a real space model corresponding to the least-squares fit of the form factor consisting of three Gaussians (symmetric bilayer profile) with Gaussian chain coronas shown in Fig. 4.3 (red lines). Figure $4.4(B)$ shows the corresponding size distribution 
Table 4.3: Parameter values with 95\% marginal confidence intervals as obtained, from the optimized anisotropic SAXS models.

\begin{tabular}{lccr}
\hline Model fit parameter & Gaussian chain coronas & Hard sphere coronas* & Unit \\
\hline$\rho_{\text {in }}+333, \rho_{\text {out }}+333$ & $379.8 \pm 1.3$ & $375.7 \pm 0.5$ & $\mathrm{e}^{-} \mathrm{nm}^{-3}$ \\
$\rho_{\text {tail }}+333$ & $304.2 \pm 3.2$ & $99.9 \pm 46.1$ & $\mathrm{e}^{-} \mathrm{nm}^{-3}$ \\
$t_{\text {in }} \sqrt{2 \pi}, t_{\text {out }} \sqrt{2 \pi}$ & $1.8 \pm 0.2$ & $2.9 \pm 0.2$ & $\mathrm{~nm}$ \\
$t_{\text {tail }} \sqrt{2 \pi}$ & $2.1 \pm 0.2$ & $0.6 \pm 0.2$ & $\mathrm{~nm}$ \\
$R_{g}^{\text {in }}$ & $3.2 \pm 0.1$ & $2.4 \pm<0.1$ & $\mathrm{~nm}$ \\
$R_{g}^{\text {out }}$ & $5.7 \pm 0.3$ & $5.6 \pm 0.1$ & $\mathrm{~nm}$ \\
$N_{c}^{\text {in }} /\left(4 \pi\left(R-D-R_{g}^{\text {in }}\right)^{2}\right)$ & $(7.09 \pm 0.99) \times 10^{-3}$ & $(13.75 \pm 0.95) \times 10^{-3}$ & $\mathrm{~nm}^{-2}$ \\
$N_{c}^{\text {out }} /\left(4 \pi\left(R+R_{g}^{\text {out }}\right)^{2}\right)$ & $(0.47 \pm 0.05) \times 10^{-3}$ & $(0.42 \pm 0.03) \times 10^{-3}$ & $\mathrm{~nm}^{-2}$ \\
$\rho_{c}+333$ & $385.1 \pm 1.5$ & $399.0 \pm 1.4$ & $\mathrm{e}^{-} \mathrm{nm}^{-3}$ \\
Mean radius larger particles & $210.1 \pm 7.2$ & $232.5 \pm 21.1$ & $\mathrm{~nm}^{3}$ \\
Width distribution larger particles & $50.2 \pm 2.8$ & $54.5 \pm<0.1$ & $\mathrm{~nm}$ \\
Number larger particles & $(0.56 \pm 0.04) \times 10^{9}$ & $(0.42 \pm 0.06) \times 10^{9}$ & $\mu 1^{-1}$ \\
Constant background & $(-14.8 \pm 2.9) \times 10^{-6}$ & $(13.5 \pm 22.6) \times 10^{-6}$ & $\mathrm{~mm}^{-1}$ \\
\hline$* R_{g}^{\text {in }}=R^{\text {in }}$ and $R_{g}^{\text {out }}=R^{\text {out }}$ & & &
\end{tabular}

Table 4.4: Properties of the best fit SV model structure with Gaussian chain coronas, $R=21 \mathrm{~nm}$

\begin{tabular}{lcr}
\hline Model property & Numerical value & Unit \\
\hline Dry mass entire SV & $32.5 \times 10^{-18}$ & $\mathrm{~g}$ \\
Dry mass lipid bilayer & $26.4 \times 10^{-18}$ & $\mathrm{~g}$ \\
Dry mass Gaussian chains inside & $2.0 \times 10^{-18}$ & $\mathrm{~g}$ \\
Dry mass Gaussian chains outside & $4.0 \times 10^{-18}$ & $\mathrm{~g}$ \\
Number Gaussian chains inside $\left(N_{c}^{\text {in }}\right)$ & 12.9 & 1 \\
Number Gaussian chains outside $\left(N_{c}^{\text {out }}\right)$ & 4.2 & 1 \\
Cross-section Gaussian chain inside $\left(R_{g}^{\text {in }}{ }^{2} \pi\right)$ & 31 & $\mathrm{~nm}^{2}$ \\
Cross-section Gaussian chain outside $\left(R_{g}^{\text {out }}{ }^{2} \pi\right)$ & 103 & $\mathrm{~nm}^{2}$ \\
Surface coverage Gaussian chains inside* & 10 & $\%$ \\
Surface coverage Gaussian chains outside* & 11 & $\%$ \\
Buoyant density entire SV & 1.05 & $\mathrm{~g} / \mathrm{ml}$ \\
\hline$*$
\end{tabular}

* projected onto $r=R_{\text {tail }}$ 
A

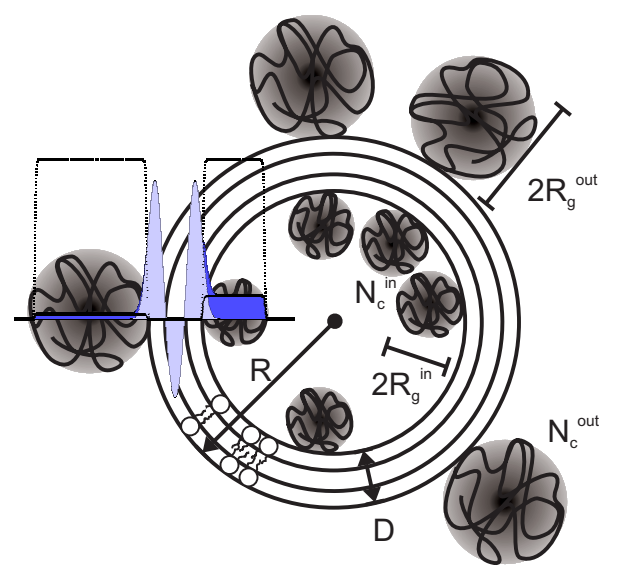

C

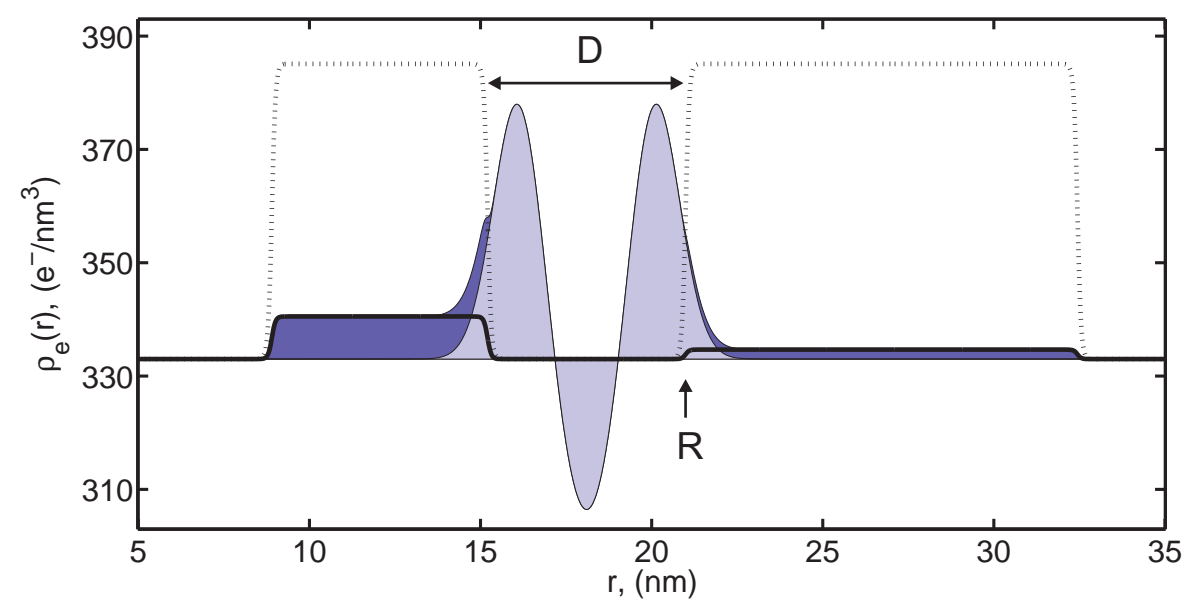

Figure 4.4: (A) Sketch of a real space model corresponding to an optimized scattering form factor consistent with the measured SAXS data from SVs shown in Fig. 4.3. For details on the model parameters see Table 4.2 (model Gaussian chains coronas) and (15). (B) Bimodal size distribution function $p(R)$ employed in the form factor calculation. Binning size $1 \mathrm{~nm}$. $(C)$ Electron density profile $\rho_{e}(r)$ across a SV membrane corresponding to least-squares fit given in Fig. 4.3 (full line red). Typical maximum local contribution of Gaussian chains (dotted line, black), and spherically averaged contribution of Gaussian chains (full line, black) are shown. Light blue area: lipid bilayer. Darker blue area: spherically averaged contribution of protein layers. Both $\rho_{e}(r)$ and $p(R)$ have been calibrated to absolute scales. Figure adapted from (15). 
function $p(R)$, and $(C)$ the electron density profile $\rho_{e}(r)$ deduced from the optimized model calculation.

The dry mass of the average SV is $32.5 \times 10^{-18} \mathrm{~g}$ which compares well to values determined by quantitative dark-field scanning transmission electron microscopy $\left((26.4 \pm 5.8) \times 10^{-18} \mathrm{~g}\right)(14)$. The total particle concentration in the sample is calculated to be $6.5 \times 10^{10}$ particles per $\mu l$ partitioned into $99.1 \% \mathrm{SVs}$ and about $0.9 \%$ larger particles. The theoretical buoyant density of a SV with a radius $R=21 \mathrm{~nm}$ is about $1.05 \mathrm{~g} / \mathrm{ml}$. The characteristic thickness of the concentric Gaussian shells is $5.7 \mathrm{~nm}$, and can be interpreted as an effective thickness of the bilayer structure of the SV. The extension of the Gaussian chains is $6.3 \mathrm{~nm}$ (facing inwards) and $11.4 \mathrm{~nm}$ (facing outwards). A SV of size $R=21 \mathrm{~nm}$ is decorated with an average of 12.9 Gaussian chains on the inside and 4.2 Gaussian chains on the outside. Projected onto the middle of the bilayer structure these cover about $10 \%$ and $11 \%$ of the surface area, respectively.

The structural parameters of the model representing the average SV structure are given in Tables 4.3 and 4.4, and confirm the values published in literature, which were derived using biochemical methods (14).

The main features of the model with attached Gaussian chains are replicated in the model with attached spheres. In particular, the parameters of the Gaussian chains and the spheres in the two models indicate that breaking of spherical symmetry is an essential ingredient needed to describe the data well (15).

\subsubsection{Model Liposomes}

Figure 4.5 shows data $I(q)$ vs $q$ from a control sample consisting of DOPS vesicles, $20 \mathrm{mg} / \mathrm{ml}$ in water (blue squares), and how they compare to SV data (black circles). While the data looks qualitatively similar towards both lower and higher q values for the measured interval, the scattered intensities differ up to approximately two orders of magnitude at intermediate $q$ values. A least-squares fit of an isotropic model (three Gaussians, symmetric bilayer profile, and mono-modal Gaussian distributed polydispersity of the particles) to the DOPS data (dashed cyan line) describes the data well (reduced $\chi^{2}=1.07$ ), although the slight lift-off of the minima towards larger $q$ values of the measured interval, which is usually due to asymmetry of the bilayer profile, is naturally not reproduced by the model calculation employed here (84). In addition to the parameter values given in Table 4.5, the fit yields for the most frequent radius $\mu=48.5 \mathrm{~nm}$, and for the width of the polydispersity distribution $\sigma=10.2 \mathrm{~nm}$. The same type of isotropic model (but with a bimodal polydispersity function) applied to the SV data (dashed dark green line) fails qualitatively to describe the data, especially in a region around $q \simeq 0.5 \mathrm{~nm}^{-1}$ where data and best-fit deviate by more than one order of magnitude. For 


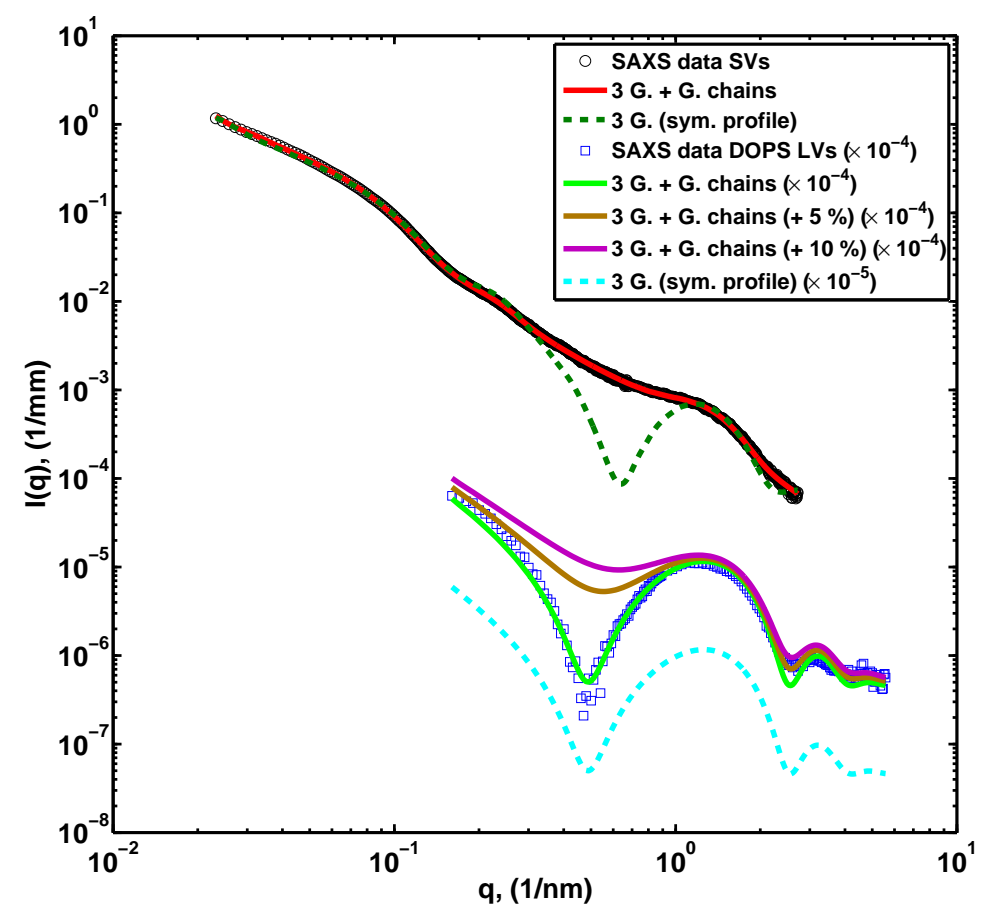

Figure 4.5: DOPS vesicle data (blue squares). Fit of isotropic model (three Gaussians, symmetric bilayer profile) to the DOPS data (dashed cyan line) and anisotropic model with Gaussian chains (full light green line). Anisotropic model with Gaussian chain contributions form SV model added ( $5 \%$, full brown line) and (10\%, full purple line). Curves partly shifted downwards for clarity, as detailed in the key. SVs (black circles), data identical to data shown in Fig. 4.3. Fit of isotropic model to the SV data (dashed dark green line) and anisotropic model with Gaussian chains (full red line). Figure adapted from (17). 
Table 4.5: Parameter values of the decorated bilayer as obtained from the optimized isotropic and anisotropic SAXS model fits shown in Fig. 4.5. Parameter values of the corresponding optimized polydispersity distributions of the SVS and DOPS LVs are given in the text.

\begin{tabular}{|c|c|c|c|c|c|c|}
\hline Sample & Model & $\begin{array}{l}\rho_{\text {in,out }}^{*} \\
\rho_{\text {tail }}^{*} \\
\end{array}$ & $\begin{array}{l}t_{\text {in }, \text { out }} \sqrt{2 \pi} \\
t_{\text {tail }} \sqrt{2 \pi}\end{array}$ & $\rho_{c}^{*}$ & $\begin{array}{l}R_{g}^{\text {in }} \\
R_{g}^{\text {out }}\end{array}$ & $\begin{array}{l}N_{c}^{\text {in }} /\left(4 \pi\left(R-D-R_{g}^{\text {in }}\right)^{2}\right) \\
N_{c}^{\text {out }} /\left(4 \pi\left(R+R_{g}^{\text {out }}\right)^{2}\right)\end{array}$ \\
\hline \multirow[t]{2}{*}{ SVs } & isotropic $\#$ & 2.29 a.u. & $1.64 \mathrm{~nm}$ & - & - & - \\
\hline & & -1 a.u. & $2.38 \mathrm{~nm}$ & & - & - \\
\hline \multirow[t]{2}{*}{ SVs } & anisotropic $\$$ & 1.63 a.u. & $1.8 \mathrm{~nm}$ & 1.81 a.u. & $3.2 \mathrm{~nm}$ & $7.09 \times 10^{-3} \mathrm{~nm}^{-2}$ \\
\hline & & -1 a.u. & $2.1 \mathrm{~nm}$ & & $5.7 \mathrm{~nm}$ & $0.47 \times 10^{-3} \mathrm{~nm}^{-2}$ \\
\hline \multirow[t]{2}{*}{ DOPS LVs } & isotropic & 2.24 a.u. & $0.89 \mathrm{~nm}$ & - & - & - \\
\hline & & -1 a.u. & $2.81 \mathrm{~nm}$ & & - & - \\
\hline \multirow[t]{2}{*}{ DOPS LVs } & anisotropic & 2.24 a.u. & $0.89 \mathrm{~nm}$ & 1.30 a.u. & $3.9 \mathrm{~nm}$ & $<1 \times 10^{-13} \mathrm{~nm}^{-2}$ \\
\hline & & -1 a.u. & $2.81 \mathrm{~nm}$ & & $4.0 \mathrm{~nm}$ & $<1 \times 10^{-13} \mathrm{~nm}^{-2}$ \\
\hline \multirow[t]{2}{*}{ DOPS LVs } & anisotropic & 2.24 a.u. & $0.89 \mathrm{~nm}$ & 2.49 a.u. & $3.2 \mathrm{~nm}$ & $3.55 \times 10^{-3} \mathrm{~nm}^{-3}$ \\
\hline & ( $+5 \%$ G. chains $)$ & -1 a.u. & $2.81 \mathrm{~nm}$ & & $5.7 \mathrm{~nm}$ & $0.24 \times 10^{-3} \mathrm{~nm}^{-3}$ \\
\hline \multirow[t]{2}{*}{ DOPS LVs } & anisotropic & 2.24 a.u. & $0.89 \mathrm{~nm}$ & 2.49 a.u. & $3.2 \mathrm{~nm}$ & $7.09 \times 10^{-3} \mathrm{~nm}^{-3}$ \\
\hline & (+10\% G. chains) & -1 a.u. & $2.81 \mathrm{~nm}$ & & $5.7 \mathrm{~nm}$ & $0.47 \times 10^{-3} \mathrm{~nm}^{-3}$ \\
\hline
\end{tabular}

* Normalized to $\rho_{\text {tail }}=-1$

\# Identical to model fit 3 Gaussians (symmetric bilayer profile) in Table 4.1.

$\$$ Identical to model fit Gaussian chains coronas in Table 4.2.

the mean of the Gaussian component of the bimodal polydispersity distribution the fit reveals $\mu=160.7 \mathrm{~nm}$, for the width $\sigma=45.2 \mathrm{~nm}$, and for the number density of large particles $1.54 \%$.

Least-squares fits of an anisotropic model, similar to the one above but with additional Gaussian chains attached to both sides of the bilayer, to the DOPS data (full light green line), yield almost identical results as the isotropic model (reduced $\chi^{2}=1.07$ ). The size distribution of the liposomes is again assumed to be Gaussian. In addition to the parameter values given in Table 4.5, for the polydispersity distribution the fit yields the most frequent radius $\mu=48.5 \mathrm{~nm}$, and a width of $\sigma=10.2 \mathrm{~nm}$. In addition, a small constant background was fitted. Interestingly, the number of Gaussian chains in the anisotropic model is zero for the best fit to the DOPS data (within the numerical precision). The qualitative effects of the attached Gaussian chains of the anisotropic model fit to the DOPS data is illustrated by adding $5 \%$ (full brown line) and $10 \%$ (full purple line) of the number of attached Gaussian chains as compared to the corresponding optimized SV model. The parameter values for $R_{g}^{\text {in }}$ and $R_{g}^{\text {out }}$ of the Gaussian chains are taken from the optimized SV model, while the parameter value for $\rho_{c}$ is calculated relative to $\rho_{\text {in }}=\rho_{\text {out }}$ to have the same value as for the optimized SV model.

A least-squares fit of the anisotropic model to the SV data (full red line, same fit as in Fig. 4.3) is in excellent agreement with the data, while employing physiologically and biochemically 


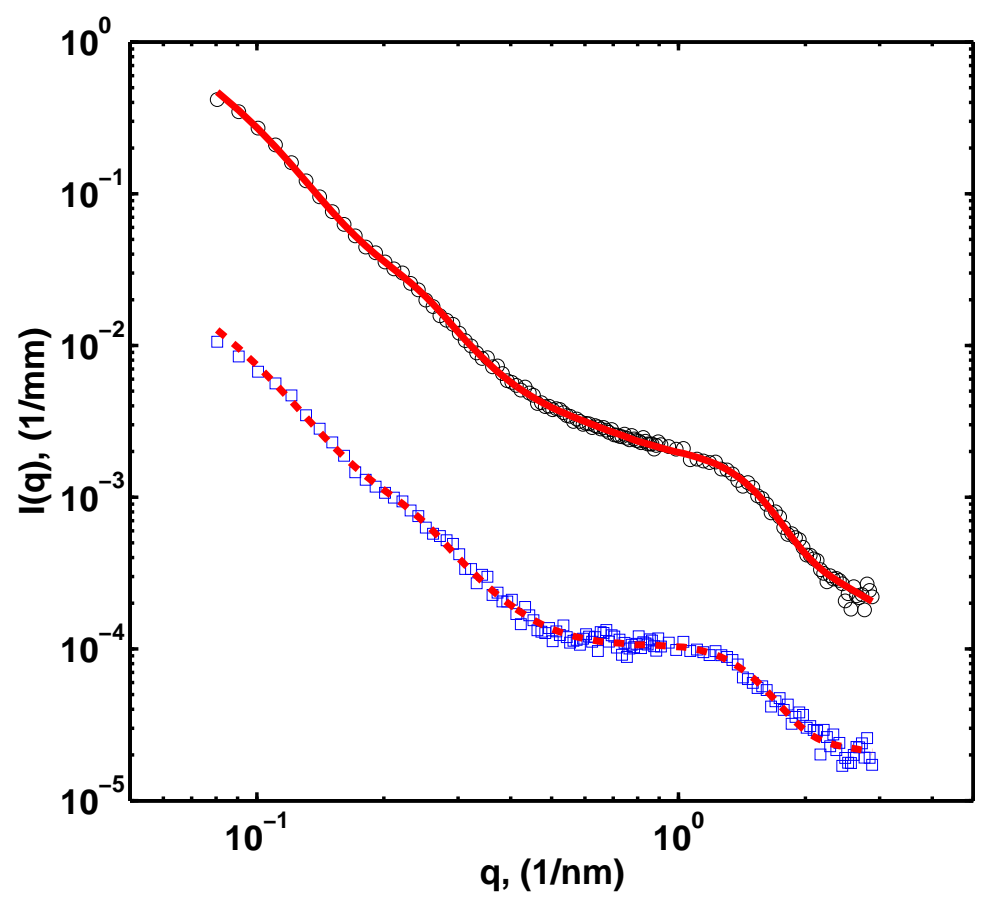

Figure 4.6: SVs treated with protease to remove protein residues facing outwards, with an additional centrifugation in purification protocol (blue squres, shifted by factor 0.1 for clarity), and. SVs only with additional centrifugation in purification protocol (black circles). Fit of anisotropic model with Gaussian chains attached to the SV data (full line red, and dashed line red, shifted by factor 0.1 for clarity). Figure adapted from (17).

meaningful parameter values $(14,15)$.

\subsubsection{Protease Treated Synaptic Vesicles}

Figure 4.6 shows the SAXS intensity function $I(q)$ vs. $q$ for a SV sample (black circles) and SV treated with the protease Trypsin (blue squres) and corresponding least-squares fits (reduced $\chi^{2}=0.66$, and reduced $\chi^{2}=0.51$, respectively) to the form factor model (solid red line, and dashed red line, respectively) for bilayer vesicles with Gaussian chains attached to the inside and outside, as detailed in section4.5. Again a bimodal size polydispersity distribution function $p(R)$ was used, with the left branch corresponding to the cryo-EM data of SVs centered around $21 \mathrm{~nm}$, and the right branch corresponding to the larger membranous particles, modeled by a Gaussian distribution centered around $\mu \approx 210 \mathrm{~nm}$ (Mean radius larger particles) with a standard deviation of $\sigma \approx 50 \mathrm{~nm}$ (Width distribution larger particles). It should be emphasized that due to the resolution of the SAXS technique, our data are relatively insensitive to the exact size distribution of these larger particles. The errors appear to slightly overestimate the actual variances, as the model fit does not over-fit the data, even though we obtain $\chi^{2}<1$ 
Table 4.6: Parameter values as obtained from the optimized anisotropic SAXS model fits shown in Fig. 4.6

\begin{tabular}{lccc}
\hline Model fit parameter & Native SVs & SVs protease & Unit \\
\hline$\rho_{\text {in }}, \rho_{\text {out }}$ & 1.58 & 1.50 & a.u. \\
$\rho_{\text {tail }}$ & -1 & -1 & a.u. \\
$t_{\text {in }} \sqrt{2 \pi}, t_{\text {out }} \sqrt{2 \pi}$ & 1.9 & 1.7 & $\mathrm{~nm}$ \\
$t_{\text {tail }} \sqrt{2 \pi}$ & 2.1 & 2.4 & $\mathrm{~nm}$ \\
$R_{g}^{\text {in }}$ & 2.8 & 2.6 & $\mathrm{~nm}$ \\
$R_{g}^{\text {out }}$ & 5.7 & 5.9 & $\mathrm{~nm}$ \\
$N_{c}^{\text {in }} /\left(4 \pi\left(R-D-R_{g}^{\text {in }}\right)^{2}\right)$ & $15.04 \times 10^{-3}$ & $8.73 \times 10^{-3}$ & $\mathrm{~nm}^{-2}$ \\
$N_{c}^{\text {out }} /\left(4 \pi\left(R+R_{g}^{\text {out }}\right)^{2}\right)$ & $0.27 \times 10^{-3}$ & $0.15 \times 10^{-3}$ & $\mathrm{~nm}^{-2}$ \\
$\rho_{c}$ & 1.84 & 1.73 & $\mathrm{a.u}$. \\
Mean radius larger particles & 210.5 & 211.0 & $\mathrm{~nm}$ \\
Width distribution larger particles & 49.7 & 51.1 & $\mathrm{~nm}$ \\
Number larger particles & 0.86 & 0.68 & $\%$ \\
Constant background & $3.67 \times 10^{-4}$ & $15.5 \times 10^{-4}$ & $\mathrm{~mm}^{-1}$ \\
\hline
\end{tabular}

for both fits. The parameters of the fits are given in Table 4.6. Most parameter values do not change much when comparing the results of the fit to the SVs (with additional centrifugation step in the purification protocol) data and the protease treated SV sample, also with additional centrifugation step. But, importantly, the number density of Gaussian chains facing the lumen and the outside are both reduced by about a factor of two as an effect of the protease treatment of the sample.

\subsection{Conclusions}

Model independent data analysis of SAXS data from SVs has been considered. However, due to inherent limitations of model independent approaches, they do not seem well suited to elucidate the complex and heterogeneous structure of SVs. Thus, in a direct modeling approach we derived and investigated different spherically symmetric and anisotropic form factor models and tested them against high resolution SAXS data from SVs, isolated from rat brain. Anisotropy of the model form factors is found to be a key ingredient for the description of the native SV structure. After protease digestion of the surface of the SVs (unspecific protein removal), a significant reduction of the anisotropic terms has been observed. This compares well with the observation that the anisotropic terms in the form factor vanish in least-squares fits of SAXS data from small unilamellar vesicles composed of DOPS. These vesicles are spherically symmetric on the experimentally resolved length scales. Besides the excellent agreement with 
the SAXS data, the suggested SV form factor model is consistent with published electron microscopy, biochemical and physiological data. Furthermore, we have presented SAXS data of SVs recorded under different $\mathrm{pH}$ conditions, and have described changes in structural parameters due to protease digestion of SVs. 


\section{Chapter 5}

\section{Model Evaluation and Structural Results}

In this chapter the models being consistent with SAXS data are interpreted and discussed. Quantitative biochemical data on the SV composition, as well as structural information on individual proteins are employed in the model analysis (Section 5.1). The size polydispersity distribution of the SVs, and the SV bilayer structure are addressed. Further, possible model dependencies and ambiguities of the results are discussed. Moreover, the SV model structure established here suggests entropic implications, which are described and investigated by employing a microcanonical model (Section 5.2). Finally, conclusions from the analysis of the structural SV model are summarized (Section 5.3).

\subsection{Model Interpretation and Discussion}

\subsubsection{Size Polydispersity}

We addressed the size distribution function (polydispersity) $p(R)$ by measuring consistent values with two independent techniques (15). The SAXS results were obtained on large ensembles and so were subject to intrinsic averaging. In contrast, with cryo-EM, micrographs of over 500 individual vesicles were taken to increase statistical relevance. Owing to the strong size dependent variation of the scattering intensity, SAXS is sensitive to larger particles, which can be minimized but which are unavoidable during sample preparation.

The resulting size distribution function $p(R)$ can thus be considered as free of artifacts related to a specific technique and presumably represents the SV structure averaged across many nerve terminals and with vesicles at all points of the cycling pathway. Some of the structural heterogeneity seen at the cryo-EM level (Fig. $2.2(B$ and $C$ )) is likely to be due to heterogeneous occupancy of the vesicle surface by accessory proteins. However, such an average is still likely to be highly informative as each vesicle contains one isoform of each of the major SV proteins responsible for vesicle function - including synaptobrevin, synaptotagmin 
and synaptophysin (14).

In light of the fact that proteins, and protein macromolecular complexes, generally display consistent sizes, the size polydispersity of synaptic vesicles (in agreement with previous electron microscopic studies of intact presynaptic terminals (85)), is surprising to us. Neurotransmitter content is lost during purification (86), so this effect is not caused by increasing osmotic pressure, with elevated neurotransmitter concentration inflating vesicles, consistent with the observation that membrane bilayers cannot be stretched by more than $3 \%$ (85). A more likely source of size variation comes from the reformation of SVs following exocytosis via clathrin mediated endocytosis. Vesicle size is presumably influenced by the size of the clathrin cage initially formed on the plasma membrane. Clathrin cage formation may be a simple stochastic process (87), or alternatively, clathrin-coat formation may be an active process where the retrieval of sufficient cargo is proof-read (88) and vesicle size can be influenced by the diffusion of synaptic proteins through the membrane following fusion (15) (see below).

What remains unclear is the effect on vesicle function (if any) produced by this polydispersity; vesicles with $R=16 \mathrm{~nm}$ and $R=24 \mathrm{~nm}$ (Fig. $2.2(A)$ ) differ by more than a factor of two in surface area and a factor of three in volume. Amongst other things, such differences in surface area and volume may have important consequences for the spatial (and functional) arrangement of trans-membrane domain proteins in the vesicle and for neurotransmitter content. It remains unclear whether the number of SV proteins scales with vesicle size. However, it is unlikely that vesicles retain a constant number of proteins during their lifetime. Recent studies suggest an exchange of proteins with the plasma membrane during exocytosis (89). We favor a model in which SV composition is effectively variable, with slight infidelities in the recycling process, which are likely to occur under conditions of intense activity, being tolerated. In this respect, the large numbers of essential trafficking proteins on an average SV are understandable as it allows for a comfortable safety margin during cycling. It needs to be remembered, however, that the concentration of neurotransmitter in the vesicle appears to be determined, to some extent by the copy number of transporters. This might be one source of variation in the post-synaptic response to single vesicle release events. An other source of variation may relate to vesicular volume - although the situation is not entirely clear and may depend on neurotransmitter type. For instance, a clear dependence on vesicle size was found for serotonin content, while no such dependence was found for glutamate (90).

After quantification of polydispersity on an absolute scale, mass on an absolute scale, $m(R)$, can be attributed to a vesicle of a given size. This calculation is based on the calibrated SAXS intensities, as well as the proportionality between scattering length density and mass density (based on a fixed stoichiometry of protons on the scale of the resolution of the experiment). Accordingly, the dry mass of a SV of radius $R=21 \mathrm{~nm}$ is $32.5 \times 10^{-18} \mathrm{~g}$ and its theoretical buoyant density is $1.05 \mathrm{mg} / \mathrm{ml}$ - lower than the value of $1.10 \mathrm{~g} / \mathrm{ml}$ determined experimentally 
by equilibrium density-gradient centrifugation (14). At present, we are unable to reconcile these two values (15).

Let us now consider the relative contributions to the measured SV SAXS curves of photons scattered from particles belonging to the two branches of the polydispersity function $p(R)$, namely SVs and larger membranous particles, which can be considered as a contamination. Importantly, the relative scattering contributions of particles, and parts of particles, of different sizes strongly depend on the considered value of $q(17)$.

The number density of the actual SVs is about 100 times higher than the number density of the larger particles. The factor of about 10 in size difference of the particles gives a factor of about 100 in favor of the larger structures, considering the relative surface areas of the particles. This is because the part of the reciprocal space studied here contains mainly information about length scales in the order of the thickness of the protein covered bilayer. This leads to approximately equal numbers of photons scattered into the measured $q$-range from particles belonging to either branch of $p(R)$. Comparing this result to the relative number of photons scattered into the entire reciprocal space ( $q$-integrated) from the two branches of $p(R)$, this means that about $99 \%$ of the scattering intensity due to the larger particles is expected to be scattered into lower $q$-regions, as compared to the $q$-interval measured here. The results of these considerations correspond well with the contributions from the two branches of $p(R)$ in the model fit calculation shown in Fig. 4.3, (red lines).

\subsubsection{Bilayer Structure}

The SAXS data presented here is, by itself, limited due to the low spatial resolution attainable with this technique. The greatest strength of our study relates to the application of independently obtained biochemical data in order to develop a coarse-grained description of the different SV constituents in relation to one another (14). To this end, we address the electron density profile $\rho_{e}(r)$ from the SAXS analysis, establishing how to interpret the Gaussian chain layers of the model (15). Since SAXS is unable to reach atomic resolution, the Gaussian chains must be considered as effective scattering centers distributed on the lipid bilayer, which can be considered to represent very large proteins or protein clusters of known mass (14). The model parameters can be interpreted beyond the total protein mass, when viewed in terms of the known protein inventory provided by biochemical analysis (14), while the (effective) lengthscale indicated by the radii of gyration $R_{g}$ of the Gaussian chains can also be postulated. According to Takamori et al., the major protein components of an average vesicle isolated from rat brain are (weight \% of total SV proteins, copy-number per SV, number of trans-membrane domains per molecule) synaptophysin $1(10.2,31.5,4)$, synaptobrevin $2(8.6,69.8,1)$, VGLUT (averaged for VGLUT1 and VGLUT2 containing vesicles - 6.0, 10.0, 10), synapsin 1 (6.0, 8.3, $0)$, synaptotagmin 1 (7.0, 15.2, 1), Rab3A $(2.5,10.3,0)$ and syntaxin 1 (2.0, 6.2, 1). These 
proteins, which are essential for both exocytosis and neurotransmitter loading, account for approximately $50 \%$ of the total SV protein inventory, corresponding to 151 individual protein molecules with a total of 319 trans-membrane domains. However, as only the dominant isoform was measured for most proteins including synaptophysin, synaptobrevin and synaptotagmin, the copy-number of each protein is likely to be higher. When taking into account other proteins, such as the V-ATPase complex and synaptogyrin, the integral membrane proteins are likely to contribute almost 600 trans-membrane domains (14). For an average $R=21 \mathrm{~nm}$ vesicle, this would equate to approximately $20 \%$ of the surface (14) in excellent agreement with the $21 \%$ surface coverage of the Gaussian chains (15).

The V-ATPase is the largest protein on the vesicle and faces outwards. Although it is only present in one or two copies, its large size means it contributes $1.2 \%$ of the total protein mass. In our model, the Gaussian chains facing outwards contribute about $12.4 \%$ of the total mass of a vesicle (see Table 4.4 ) and thus about $21.1 \%$ of the total protein mass (14). At most $5.7 \%$ of the mass represented by the outward facing Gaussian chains can be attributed to the V-ATPase. The remaining $94.3 \%$ of the protein mass in the Gaussian chains cannot be attributed to individual protein components. First, the individual copy-numbers would be too low. Second, the lateral extension would be too large. While extended proteins such as synaptobrevin could account for an $2 R_{g}^{\text {out }}=11.4 \mathrm{~nm}$ extension in the radial direction (91), the corresponding lateral size indicates a clustering of proteins into domain structures as there are too few (known) large proteins on the vesicle that match the size of the Gaussian chains in the 'in-plane' direction (15). Interestingly, cholesterol-dependent clustering of the synaptic vesicle proteins synaptobrevin, synaptotagmin and synaptophysin into domains has been reported in a crude synaptic vesicle preparation (92), suggesting that the fusion apparatus might be concentrated in a specialized membrane patch. This clustering might have important consequences for vesicle function. For instance, the vesicular SNARE protein synaptobrevin, which interacts with syntaxin 1 and SNAP-25 in the plasma membrane to initiate fusion, has a cytosolic length of approximately $10 \mathrm{~nm}$, as do syntaxin and SNAP-25. Given that SNARE interactions are initiated N-terminally and proceed towards the $\mathrm{C}$ terminus in a zipper-like fashion, thus pulling the membranes together, it is likely that SNARE initiation can proceed from a distance of up to $20 \mathrm{~nm}$ from the plasma membrane, a value consistent with that proposed by single vesicle tracking experiments in living neurons (91).

\subsubsection{Model Dependencies and Ambiguities}

In combination with complimentary techniques such as cryo-EM and biochemical analysis, we have shown that SAXS is capable of elucidating structures of significant complexity such as synaptic vesicles.

State of the art beamlines have been used to record the SAXS data, which were found to be 
highly reproducible (15). As we show in Fig. 4.3, several different isotropic models have been discussed and have been falsified. After assessment of numerous samples, we are convinced that the presented effective model representing proteins in a coarse-grained sense by Gaussian chains is well suited to describe the synaptic vesicles quantitatively at the given resolution.

However, possible ambiguities and model dependencies of the results are important issues which need to be addressed explicitly. We have therefore compared fits with two independent anisotropic models that share the main features of anisotropic protein clusters but use different detailed implementations $(15,17)$. In particular, the clustered proteins are represented by hard spheres in the first, and by Gaussian chains in the second. Importantly, these two models show qualitatively similar results, and thus support the main conclusion that an anisotropic model is needed to accurately describe the SV data. Of course, to some extent the derived model parameters of the observed clustering effect are different. These differences mark the degree to which the results depend on the specific formulation of the model, see Table 4.2. The main conclusions and existence of protein clusters are, however, maintained in a model independent way.

Models where the proteins are represented by spherically symmetric Gaussian electron densities as depicted in Fig. 4.5 illustrate the effects that the Gaussian chains parameters, or hard spheres parameters, have on the scattering curve. In an intermediate $q$-range, the difference in scattering length of the SV data, when compared to unilamellar DOPS vesicles, is found to be in excess of two orders of magnitude (17).

Importantly, least squares model fits to the DOPS data yield almost identical results for a spherically symmetric model with a symmetric density profile of three coupled Gaussians (Fig. 4.5, dashed cyan line), and a model with Gaussian chains attached to the profile (Fig. 4.5, solid light green line). Interestingly, the number of Gaussian chains in the later model is zero for the best fit (within the numerical precision). Models with significant amounts of attached Gaussian chains with parameter values similar to the ones from the corresponding optimized SV model can be falsified against the DOPS data (Fig. 4.5, solid brown line, and solid purple line).

The mathematical model utilized to fit the experimental data contains 12 parameters to account for the structural complexity of the SV sample. Given the high complexity of the system, the high number of data points (up to 763) and the wide $q$-range of the data, a model form factor fit with 12 parameters is perfectly justified. We have verified that the model components are both necessary and sufficient to accurately describe the data, see also the reduced model fits, as well as the alternative model fits in Fig. 4.3 and Fig. 4.5. None of the contributions are marginal, neither within the model structure of one particle, nor considering the two branches of the polydispersity distribution $p(R)$.

In addition, the values for several parameters can be constrained fairly well due to infor- 
mation from cryo-EM and additional biochemical knowledge. The fact that SVs are known to have a unilamellar bilayer structure with incorporated and attached proteins introduces several constraints to the model parameters. Further, the size distribution of the larger particles can be estimated, at this point only qualitatively, by cryo-EM. The investigated $q$-range is not very sensitive to the precise size of particles in the size range of the larger particles. Due to the heterogeneous nature and broad size distribution of these particles, pronounced features at lower q regions cannot be expected. Particular features outside the measurement interval do not appear in the model calculations, either at small or at high q, i.e. the measurement range (after stitching different detector distances) was well adapted. In particular, the heterogeneous nature and broad size distribution of the larger particles suppressed any pronounced features at lower $q$-ranges.

\subsection{Model Entropy Considerations}

Protein clusters on the SV membrane indicate a state of higher ordering and thus a smaller value for the entropy when compared to a SV with proteins being randomly distributed over $4 \pi$. Thus, energy would be gained by cluster disintegration, a process which might take place upon fusion of the SV with the plasma membrane and might provide energy needed for driving the membrane fusion. Such processes might be related to membrane curvatures, too.

To estimate the entropy gain due to disintegration of clusters on the SV, a microcanonical model of the protein decorated SV membrane is investigated.

\subsubsection{Cluster Formation and Disintegration}

The proteins on the average SV are organized in clusters, forming micro-domains on the SV, consisting of protein trans-membrane domains (TMDs) (15, 17). Some proteins may remain clustered (93) after fusion of the SV with the plasma membrane of the presynaptic cell, and corresponding micro-domains may persist at least partially on the plasma membrane.

However, it seems likely that at least part of the protein micro-domains disintegrate at least partially upon fusion of the SV with the plasma membrane. Disintegration of protein clusters leads to an increase of the number of possible distinguishable states of the different lipids and proteins on the SV, and thus to an increase of entropy and a gain of free energy.

Here we speculate that the gain in free energy due to possible disintegration of protein clusters on the SV upon fusion of the SV with the plasma membrane might provide energy necessary to drive the fusion reaction of the SV membrane with the plasma membrane. Disintegration might also be energetically advantageous due to different membrane curvatures of the SV membrane and the plasma membrane, considering possible differences in the intrinsic curvatures of clustered proteins and freely diffusing proteins in the SV, or in the plasma membrane. 
Here, we estimate the expected gain in free energy due to disintegration of protein clusters by employing a simple model calculation.

\subsubsection{Model}

The entropy of the SV is calculated in a micro-canonical approach by modeling the SV by a system composed of $n_{L D}$ independent and indistinguishable lipids in each membrane leaflet, or lipid dimers facing each other with their tails, $n_{T M D}$ independent and indistinguishable free protein TMDs (not clustered), $n_{S C}$ independent and indistinguishable small protein clusters, and $n_{L C}$ independent and indistinguishable large protein clusters, according to the SV model structure given in Table 4.4 in section 4.6. The number of TMDs per protein cluster is calculated by assuming an area of $1.50 \mathrm{~nm}^{2}$ per TMD (as estimated from the tree-dimensional structure of the helical membrane proteins aquaporin-1 and $\mathrm{ClC}$ chloride channel (14)), yielding approximately 21 TMDs per small cluster and approximately 68 TMDs per large cluster. The number of lipid molecules in one leaflet is calculated to be approximately 5004 by assuming an area of $0.65 \mathrm{~nm}^{2}$ per lipid (94), and considering that $79 \%$ of the surface area of a sphere with a radius of $18.1 \mathrm{~nm}$ (middle of bilayer for most frequently occurring SV radius) are covered by lipids. The total number of TMDs in the protein clusters is calculated to be 541, assuming 13 small clusters and 4 large clusters composed of TMDs, which compares well with the value of approximately 600 TMDs per SV, as suggested on the basis of biochemical data (14). Note that the number of clusters assumed here has been obtained by rounding the numbers given in Table 4.4 to natural numbers.

The number of different states $\Omega$ considering the number of distinguishable possibilities to distribute $n_{L D}$ lipids, $n_{T M D}$ free TMDs, $n_{S C}$ small clusters, and $n_{L C}$ large clusters to $n_{B O X}$ distinguishable boxes, with $n_{B O X}=\sum_{i} n_{i}$ and $i=L D, T M D, S C, L C$, is given by

$$
\Omega=\frac{\left(\sum_{i} n_{i}\right) !}{\prod_{i}\left(n_{i}\right) !},
$$

with $i=L D, T M D, S C, L C$.

The entropy $S$ is defined by

$$
S=k_{B} \ln \Omega,
$$

with the Boltzman constant $k_{B} \approx 1.314 \times 10^{-23} \mathrm{~J} / \mathrm{K}$. Further, considering the Helmholtz potential $F \equiv U(T)=U-T S$, the difference of free Energy $\Delta U$ in units of $k_{B} T$ for a system in two states i and $\mathrm{f}$, with a difference of entropy $\Delta S=S_{f}-S_{i}$ is given by

$$
\Delta U=\frac{\Delta S}{k_{B}}
$$


The numerical calculations were performed in MATLAB. The Stirling approximation $\ln (M !) \approx$ $M \ln (M)-M$ was employed for $M>20$ for the calculation of $\ln (M !)$. Further details on the MATLAB code are given in the appendix.

\subsubsection{Results}

Complete disintegration of one of the 4 large clusters yields a system with $n_{L C}=3, n_{T M D}=68$, $n_{S C}=13$ (unchanged), $n_{L D}=5004$ (unchanged), and $n_{B O X}=5088$ (as compared to $n_{B O X}=$ 5021 for the initial system). The system has gained free energy in the order of $\Delta U \approx 354 k_{B} T$. Correspondingly, complete disintegration of one of the 13 small clusters would give about $\Delta U \approx 130 k_{B} T$ to the system. Complete disintegration of all clusters yields a system with $n_{L C}=n_{S C}=0, n_{T M D}=541, n_{L D}=5004$ (unchanged), and $n_{B O X}=5545$, which has gained free energy in the order of $\Delta U \approx 1654 k_{B} T$.

\section{Discussion and Conclusions}

Formation or breakage of a covalent bond costs in the order of $|\Delta U| \approx 100 k_{B} T$. Thus, the calculated free energy gain of $\Delta U \approx 354 k_{B} T$ due to disintegration of one of the large protein clusters on the SV corresponds to the energy needed to break up approximately 4 covalent bonds, and the disintegration of one small cluster corresponds to the energy needed to break up approximately 1 covalent bond. The calculated free energy due to total disintegration of all clusters corresponds to the energy needed to break up approximately 17 covalent bonds.

Thus this very simplistic model calculation supports the speculation, that free energy gained due to an increase of entropy on the SV might influence the fusion reaction significantly. The entropy gain would follow from the disintegration, or partial disintegration, of protein clusters on the SV upon fusion of the SV with the plasma membrane.

In conclusion, the hypothesis that the fusion reaction of the SV with the plasma membrane is significantly influenced by entropic effects related to ordering and disordering of proteins on the SV cannot be rejected on the basis of the considered model.

\subsection{Conclusions}

The scattering model used here is in excellent agreement with the SAXS data, using parameters that are consistent with published electron microscopy, biochemical and physiological data. At the same time the resulting model was obtained independently of other analytical techniques. This study thus confirms pre-existing ideas about the main structural features of SVs and adds important refinements, such as the presence of protein micro-domains, indicating a state of higher ordering and thus a smaller value for the entropy when compared to a SV with proteins 
being randomly distributed over $4 \pi$. Free energy gained due to an increase of entropy on the SV related to disintegration, or partial disintegration, of protein clusters on the SV might influence the fusion reaction significantly. To the best of our knowledge, this is the first time SAXS has been successfully applied to a functional (heterogeneous) organelle and raises the distinct possibility that SAXS analysis (when applied in combination with other analytical approaches) will provide a useful means to analyze other biological membranes. 


\section{Chapter 6}

\section{Towards Cell Free Fusion Systems}

This chapter describes ex vivo fusion systems which employ native synaptic vesicle organelles isolated from rat brain, and small unilamellar proteo-liposomes with reconstituted t-SNARE membrane proteins. Recent results from interaction and fusion systems consisting of native SVs or proteo-liposomes interacting with a planar lipid Langmuir film at the water-air interface, or a solid supported lipid bilayer are briefly reported and discussed (Section 6.1) and the sample preparation of proteo-liposomes is addressed (Section 6.2). We show that the size increase due to fusion of proteo-liposomes with native SVs can be quantified by dynamic light scattering (Section 6.3). A Gedankenexperiment reveals that SAXS seems likely to resolve details of the structural changes associated with fusion of native SVs with proteo-liposomes (Section 6.4).

\subsection{Introductory Considerations}

Cell free fusion systems provide the means to study the principles of membrane interaction and fusion at systems with reduced complexity when compared to living cells (95). The fusion systems consist of reconstituted membranes, and/or may employ native membrane structures isolated from living cells or animals (96). Further, physical and chemical conditions of cell free fusion systems can be tightly controlled and manipulated allowing to study fusion under different physiological and pathological conditions. Thus, cell free fusion systems are important tools to elucidate the physical and biochemical principles of membrane trafficking in cells.

In recent studies, we investigated cell free systems where the target membrane is represented or mimicked by a solid supported lipid bilayer, or a lipid Langmuir film at the air-water interface (Sajal Kumar Ghosh ${ }^{1}$, et al. (18)). SVs isolated from rat brain are added and interact with the solid supported bilayer in aqueous buffer solution, or SVs are injected into the aqueous subphase and interact with the lipid monolayer.

Figure 6.1 (A) shows a cartoon illustrating a SV interacting with a lipid Langmuir film. The

\footnotetext{
${ }^{1}$ Institut für Röntgenphysik, Georg-August-Universität Göttingen
} 



Figure 6.1: Model of the experimental system. (A) SVs injected in the subphase of a lipid monolayer formed at the air-water interface. (B) SVs pipetted on top of a lipid bilayer formed on a solid support. The respective electron density profiles $(\rho(z))$ of the lipid monolayer and the bilayer are also shown in $(A)$ and $(B)$, the z-axis being parallel to the sample normal. $\tau$ is the tilt angle of the lipid molecules in the gel phase. Figure adapted from (18).

SVs are added into the subphase after the preparation of the lipid Langmuir film. (B) shows a cartoon illustrating a SV interacting with a solid supported lipid bilayer. The SVs are added to the aqueous buffer covering the lipid bilayer.

Both the solid supported bilayer and the Langmuir film exhibit a planar geometry. Thus, their structure can be probed with high spatial resolution by x-ray reflectivity, and grazing incidence diffraction (GID). Using these systems, we investigated the effects of the lipid phosphatidylinositol 4,5-bisphosphate $\left(\mathrm{PIP}_{2}\right)$ on membrane interaction and fusion when added to the solid supported bilayer, or the lipid Langmuir film (18).

Figure 6.2 shows electron density profiles parallel to the sample surface normal obtained by fitting box-models to Fresnel normalized x-ray reflectivity data from solid supported bilayer systems and Langmuir films, in the absence or presence of SVs. (A) DOPC bilayer (solid red line) and DOPC bilayer with added SVs (dashed black line), both electron density profiles obtained by fitting a six box-model to the reflectivity data. (B) DOPC/PIP 2 (4:1) bilayer (solid red line, six-box model) and DOPC/PIP 2 (4:1) bilayer with added SVs (dashed black line, eight box-model). (C) DPPC Langmuir film (solid red line) and DPPC Langmuir film with added SVs (dashed black line). (D) DPPC/PIP 2 (5 mol \%) Langmuir film (solid red line) and DPPC/PIP 2 (5 mol \%) Langmuir film with added SVs (dashed black line). SVs were injected into the aqueous sub-phase of the Langmuir film, which was at a surface pressure of $30 \mathrm{mN} / \mathrm{m}$. Two-box models were fitted to the monolayer reflectivity data sets; one box corresponding to the head-group region, and one box corresponding to the tail region.

We found that SVs interact with the planar lipid systems, and the interaction is significantly enhanced by the presence of $\mathrm{PIP}_{2}$. The interaction can be further enhanced by addition of 

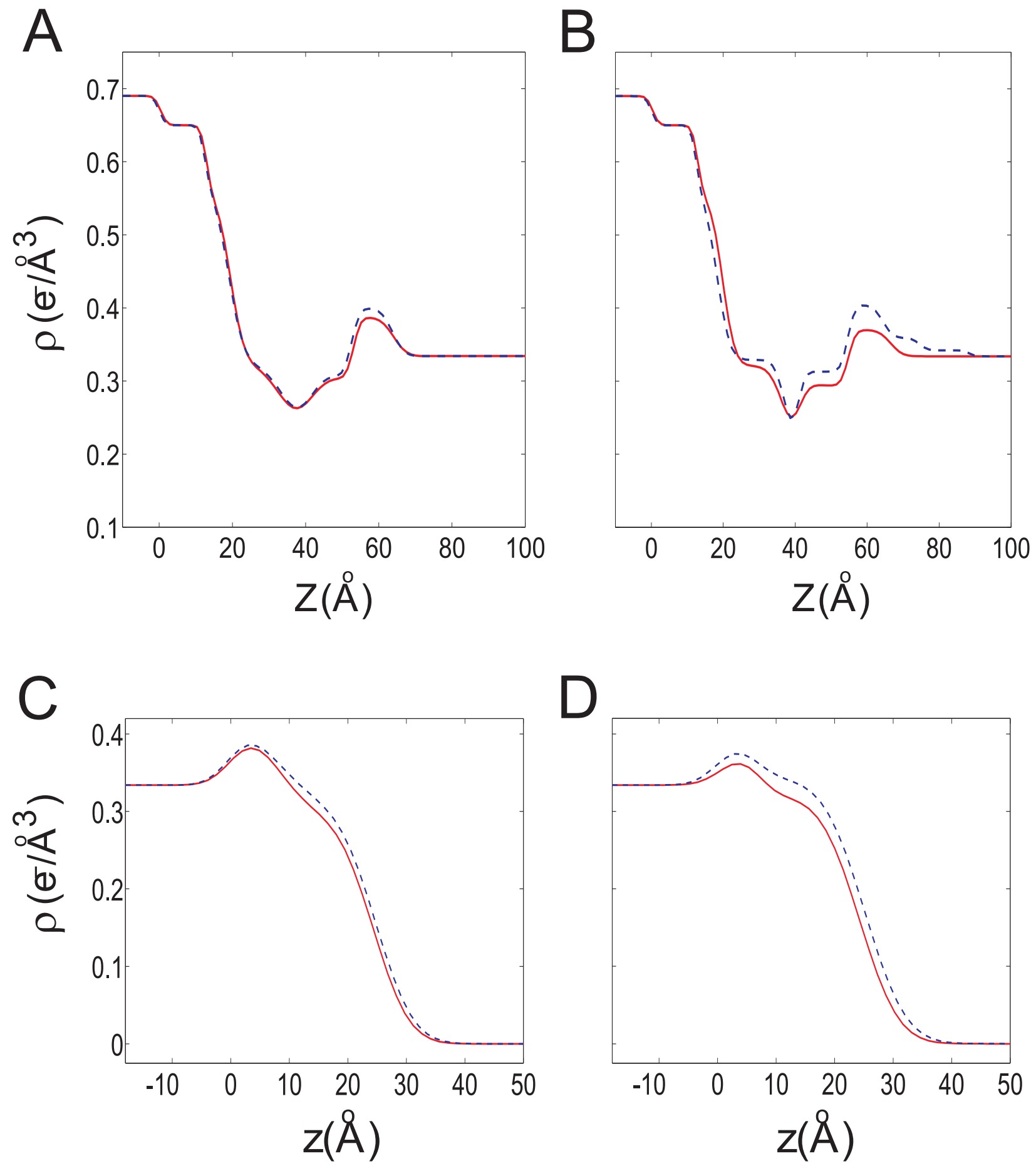

Figure 6.2: Electron density profiles obtained by fitting box-models to Fresnel normalized x-ray reflectivity data. (A) DOPC bilayer (solid red line) and DOPC bilayer with added SVs (dashed black line). (B) DOPC/PIP 2 (4:1) bilayer (solid red line) and $\mathrm{DOPC} \mathrm{PIP}_{2}$ (4:1) bilayer with added SVs (dashed black line). (C) DPPC Langmuir film (solid red line) and DPPC Langmuir film with added SVs (dashed black line). SVs were injected into the aqueous sub-phase of the Langmuir film. (D) DPPC/PIP 2 (5 mol \%) Langmuir film (solid red line) and DPPC/PIP 2 (5 mol \%) Langmuir film with added SVs (dashed black line). SVs were injected into the aqueous sub-phase of the Langmuir film. Figure adapted from (18). 


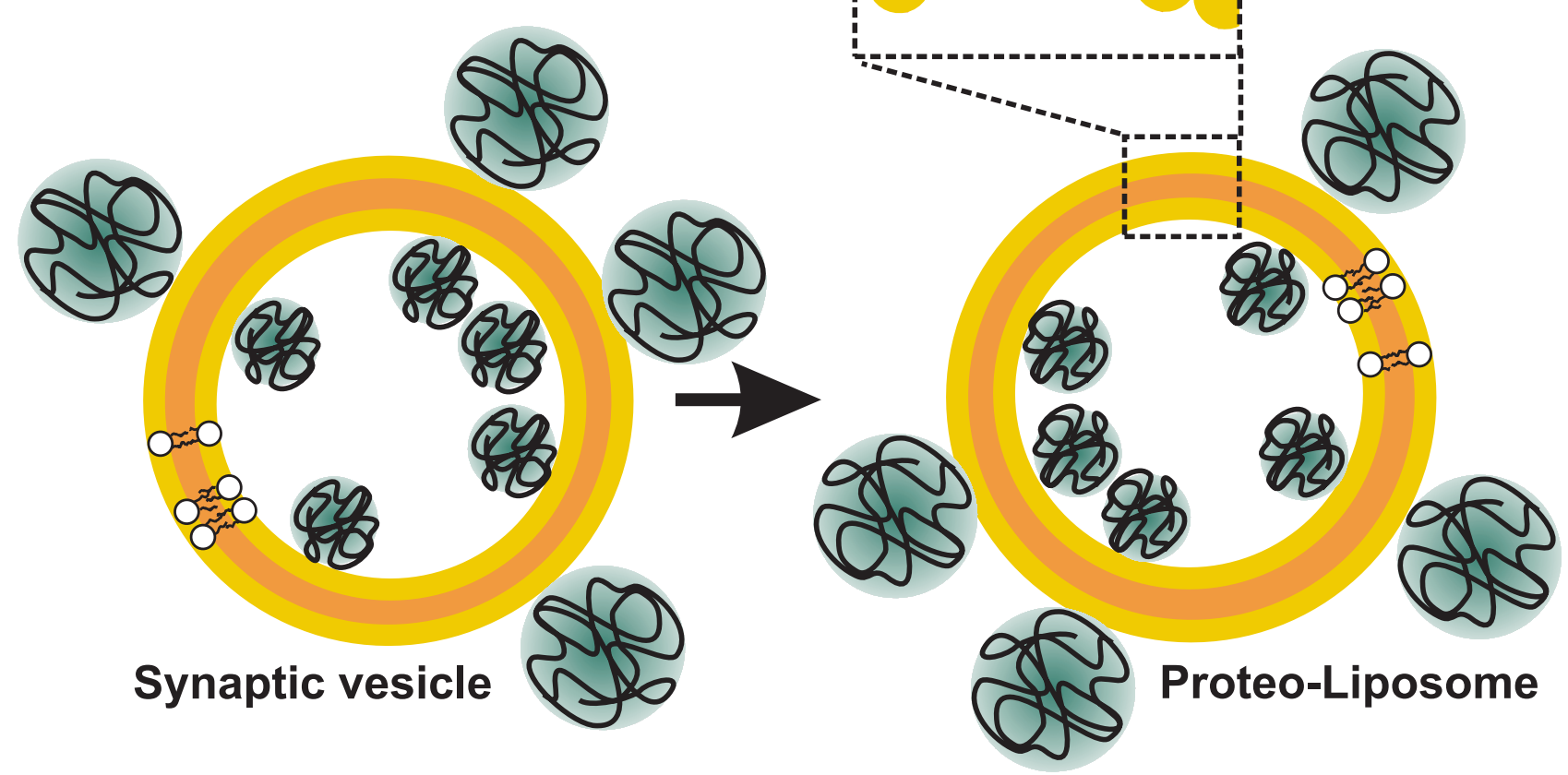

Figure 6.3: The model experimental system. Synaptic vesicles (SVs) upon interacting with small unilamellar model proteo-liposome (PL). Figure adapted from (18).

physiologically meaningful amounts of $\left[\mathrm{Ca}^{2+}\right]$ (18). A characteristic thickening of the lipid bilayer, and an increase of electron density of the upper lipid leaflet were observed for the solid supported bilayer system. Correspondingly, a characteristic thickening of the lipid Langmuir film, and an increase of the electron density of the head region were observed for the lipid monolayer system. Thus, we showed that x-ray reflectivity and GID have great promises studying the interaction of trafficking organelles with their target membranes in cell free systems (18).

However, the used planar lipid systems mimicking the SV target membrane have shortcomings, and questions remain to which extent these systems mimic the actual physiological processes present in vivo. For example, the lipid Langmuir film can only mimic one leaflet of a target membrane in vivo, and there are concerns that the behavior of the solid supported bilayer may be biased by the solid support, which may be decreased but not totally eliminated by employing soft cushions underneath the bilayer (97).

Other cell free fusion systems do not exhibit these shortcomings as the target membrane is represented by the lipid bilayer of a small unilamellar vesicle, dispersed in aqueous buffer. Membrane proteins can be reconstituted into the lipid bilayer of the lipid vesicles (proteoliposomes). 
Figure 6.3 shows a cartoon illustrating a SV interacting with a small unilamellar lipid vesicle (proteo-liposome) with reconstituted membrane proteins, depicted by spherical blobs (blue).

Here we address a cell free fusion system where SVs isolated from rat brain (14) interact, and eventually fuse with small unilamellar lipid vesicles with reconstituted t-SNARE proteins Syntaxin 1 and SNAP-25A (proteo-liposomes) (96), (Section 6.2). We show that the size increase and the number of fusion events of proteo-liposomes fusing with SVs can be quantified by DLS by determining the size distribution function of the individual particles before fusion, and of the fusion products (Section 6.3). Further, we present calculated scattering curves revealing the applicability of SAXS to study the fusion pathway of SVs and proteo-liposomes directly on a structural level (Section 6.4). Further, conclusions resulting from the DLS experiments, and the calculated SAXS curves are summarized (Section 6.5).

\subsection{Samples}

Small unilamellar proteo-liposomes (PLs) were prepared by Matthew Holt ${ }^{1}$ by mixing lipids in chloroform giving molar ratios (given in brackets) resembling that of native biological membranes according to (14), i.e. phosphatidyline (5), phosphatidylethanolamine (2), phosphatidylserine (1), phosphatidylinositol (1). Unlabeled phosphatidyl-ethanolamine (PE) was partly substituted by N-(7-nitro-2,1,3-benzoxadiazol-4-yl) PE (NBD-PE) and Rhodamine-PE (lipid dequenching), since the same samples were used in fluorescence-based assays, too (96). After dyeing, lipids were resuspended in HB100 buffer $(100 \mathrm{mM} \mathrm{KCl,} 1 \mathrm{mM}$ DTT, $25 \mathrm{mM}$ HEPES [pH 7.4, KOH]) with $5 \%$ sodium cholate at a total lipid concentration of $13.5 \mathrm{mM}$. SNARE proteins (Syntaxin 1 and SNAP-25A) were cloned with rat (Rattus norvegicus) sequences as templates and expressed in E. coli, and subsequently purified (98). SNARE proteins were added at a physiologically relevant SNARE to phospholipid molar ratio of 1:300 (14) to the cholate micellar solution. Detergent was removed by gel filtration chromatography on a SMART system with a PC 3.2/10 Fast Desalting column (GE Healthcare). For further details on the proteoliposome preparation see (96) and corresponding on-line supplemental data. Synaptic Vesicles were purified by Matthew Holt ${ }^{1}$ from rat brain, following the procedure described before, see section 2.3 .

\subsection{Dynamic Light Scattering}

We show that DLS is capable to detect and quantify the size increase of small unilamellar proteo-liposomes (PLs) which are fused in a SNARE dependent manner with SVs forming a prototypic cell free model fusion system (16).

\footnotetext{
${ }^{1}$ Department of Neurobiology, Max Planck Institut für Biophysikalische Chemie, Göttingen, Germany
} 


\subsubsection{Experiments}

SV and PL dispersions were diluted and filled in glas cuvettes as described above, see section 2.6. For the fusion experiments, a SV stock solution of $4.56 \mathrm{mg} / \mathrm{ml}$ protein concentration is diluted with degassed aqueous buffer (see above) to a total protein concentration of $0.83 \mu \mathrm{g} / \mathrm{ml}$. For the fusion reactions, Syntaxin 1/SNAP-25 proteol-iposomes (NBD-Rhodamine labeled) are added to the SV solutions at similar total mass concentration as the SVs (96), leading to particle number ratio of about 1:1. Fusion activity was checked in a similar manner like in (96) by fluorescence dequenching assays, and the size increase of the fused SV/liposomes as determined by cryo electron microscopy (data not shown). For the inhibited fusion reactions, SVs are incubated for $60 \mathrm{~min}$ at $37^{\circ} \mathrm{C}$ in $\mathrm{HB} 100$ with Tetanus toxin (TeNT), which targets Synaptobrevin, in a molar ratio of 1:50 with regard to the concentration of Synaptobrevin (96). TeNT was expressed by Matthew Holt ${ }^{2}$ in bacteria and purified with Ni2+-NTA chromatography $(99,100,101)$. Concentrations were determined with UV absorption, and proteolytic activity was tested immediately before use in experiments (96). Control samples were incubated under similar conditions in HB100 (no addition of TeNT). Absence of fusion activity for the samples of the inhibited fusion reaction, and the control samples, was checked by fluorescence dequenching assays and cryo-EM (data not shown).

The SV particle number was calculated based on the protein mass of the SVs in the sample, assuming monodisperse SVs with a radius $R=21 \mathrm{~nm}$. Particle numbers for the PLs were calculated from the total lipid mass, assuming 100\% efficiency in PL formation, and a monodisperse population with radius $R=21 \mathrm{~nm}$.

\subsubsection{Results}

Figure 6.4 shows the size distribution functions $\mathrm{p}(\mathrm{R})$ from ensembles of PLs (blue squares, solid line), native SVs (red circles, solid line) as well as the product of a fusion reaction between PLs and SVs (cyan triangles, dashed line) and a corresponding control (magenta crossed, dashed line) with TeNT treated SVs added to PLs, as obtained by DLS. The data has been analyzed using the ALV software, employing zero order regularization and describing the particles as hard spheres.

The normalized size distribution functions $p_{n}(R)$ of all four samples are similar in shape and width. The most frequent radii of both the PL and SV samples are about $21 \mathrm{~nm}$. The size distribution of the fusion products is shifted towards larger radii (maximum around $31 \mathrm{~nm}$ ) when compared to the size of the SV or PL ensembles. As expected, TeNT treatment of the SVs decreases this shift significantly (maximum around $24 \mathrm{~nm}$ ), effectively inhibiting fusion between SVs and PLs.

\footnotetext{
${ }^{2}$ Department of Neurobiology, Max Planck Institut für Biophysikalische Chemie, Göttingen, Germany
} 


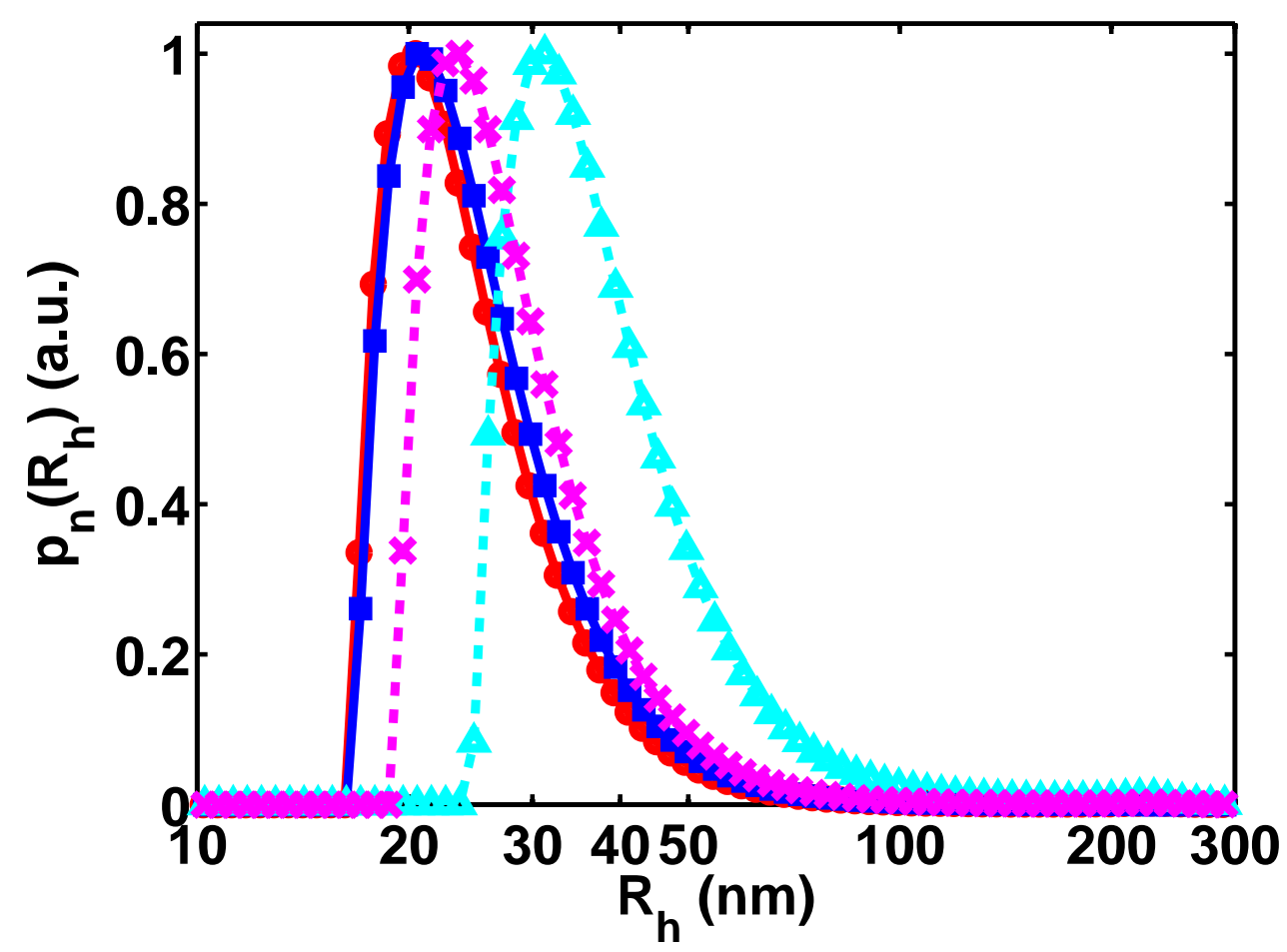

Figure 6.4: DLS data of native SV ensemble (red circles, solid line), PL preparation (blue squares, solid line), mixture of SVs plus PLs (cyan triangles, dashed line) and TeNT treated SVs plus PLs (magenta crosses, dashed line). 
Table 6.1: Model parameters as obtained from the fusion experiments by inversion of DLS data. Most frequent radius $\mathrm{R}(\mathrm{nm})$ of particles. From $\Delta \mathrm{R}$ a characteristic number of fusion events $\mathrm{N}$ (per PL) is calculated by assuming conservation of particle surface areas, and size homogeneity of SVs, PLs and fusion products, respectively.

\begin{tabular}{lcc}
\hline Sample & $\mathrm{R}(\mathrm{nm})$ & $\mathrm{N}($ per PL) \\
\hline SVs & 21 & - \\
PLs & 21 & - \\
SVs + PLs & 31 & 1.2 \\
SVs + PLs + toxin & 24 & 0.3 \\
\hline
\end{tabular}

Table 6.1 gives the most frequent radii and a characteristic number N (per PL) of fusion events of PLs and SVs, calculated from the shift of the most frequent radius when compared to the PL sample.

\subsubsection{Conclusions}

The typical size increase of small unilamellar proteo-liposomes with reconstituted SNARE proteins exhibited after SNARE mediated fusion with SVs, can be resolved and quantified by DLS (Fig. 6.4). Scattering techniques allow to obtain information averaged over a fairly large number of particles as compared to single particle imaging techniques like cryo-EM in reasonably short times (here in the order of $100 \mathrm{~s}$ ). It seems feasible to significantly increase time resolution reaching values in the order of a few seconds. The needed sample volumes are relatively small (about $1 \mathrm{ml}$ with an concentration of about $1 \mu \mathrm{g} / \mathrm{ml}$ ) and the sample can be reused.

\subsection{Small-Angle X-Ray Scattering}

\subsubsection{Gedankenexperiment}

SAXS has opened up the possibility to study SV ensembles structurally under quasi-physiological conditions $(15,17)$, avoiding invasive sample preparation steps needed for other techniques such as electron microscopy. It appears that SAXS is suited to study structurally the fusion process of SVs with model membrane systems, mimicking the synaptic membrane in neurons, such as liposomes or proteo-liposomes (PLs). Thus, we consider here calculated scattering curves and how they compare to SAXS data from SVs to illustrate the expected outcomes of Gedankenexperiments, where SVs and PLs are mixed together and eventually fuse to form hybrid SV-PL vesicles (18). Conservation of SV and PL surface area is assumed for the fusion process. Further it is assumed that all particles of the two initial polydisperse populations fuse, and that fusion probabilities are independent of the sizes of the fused particles, i.e. the 

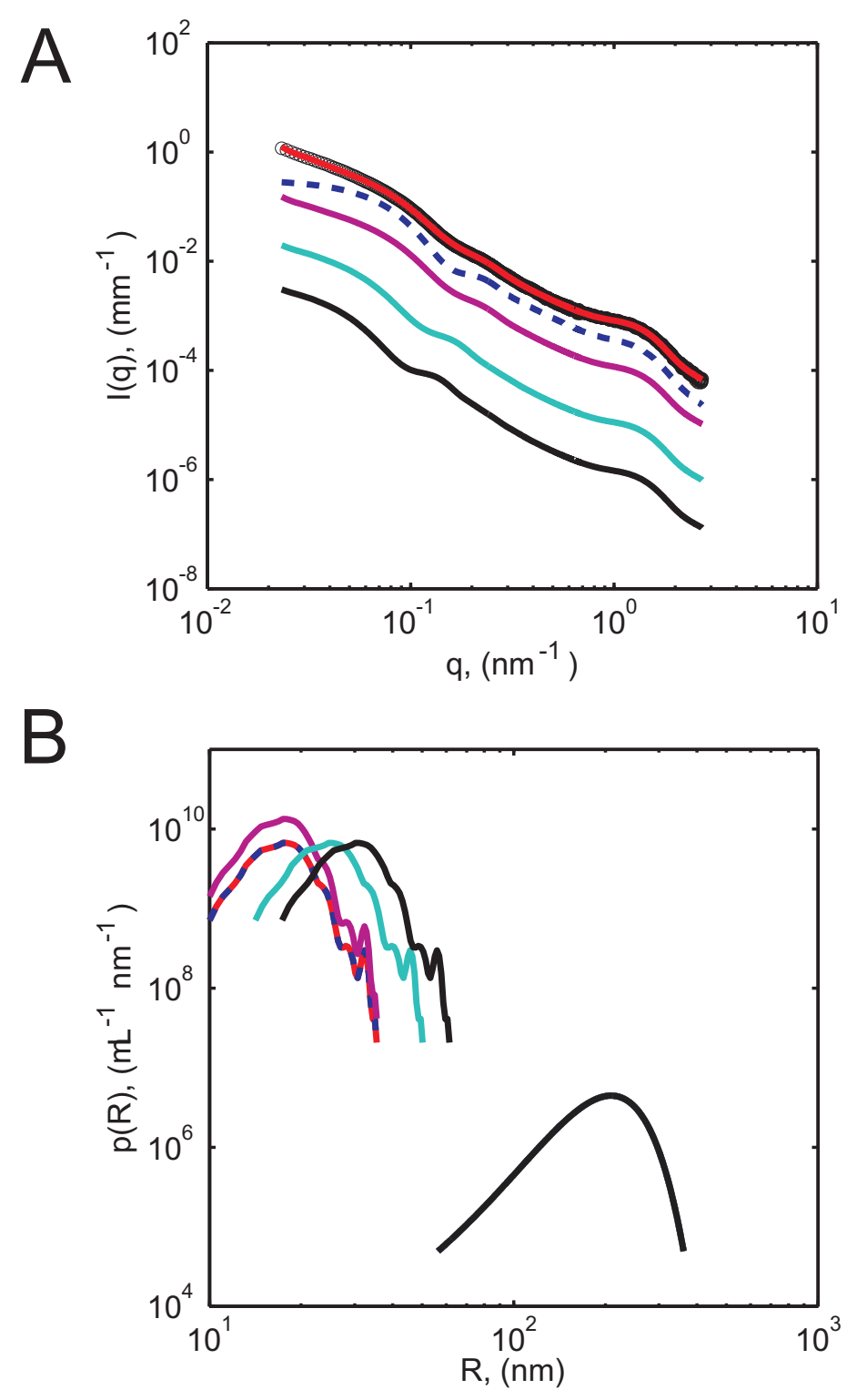

Figure 6.5: (A) SAXS data from SVs (open circles, black, $\times 10^{0}$ ), model fit to the SAXS data (full line, red, $\times 10^{0}$ ), and calculated scattering curves of proteo-liposomes (PLs) (dashed line, blue, $\times 10^{0}$ ), of a mixture of non-interacting SVs and PLs (full line, violet, number ratios 1:1, $\times 10^{-1}$ ), of SVs fused with PLs (full line, green, number ratios $1: 1, \times 10^{-2}$ ), and SVs fused with PLs (full line, black, number ratios $1: 2, \times 10^{-3}$ ). (B) Size distributions employed in the model calculations depicted in $(A)$. The right hand part of the size distributions plotted in black is part of all size distributions depicted by solid lines (full line), i.e. all size distributions are bimodal, except the case of the pure PLs (dashed line, blue). Figure adapted from (18). 
population of the fused hybrid particles (fusion product) of the two polydisperse populations will exhibit, in general, a larger polydispersity when compared to the polydispersities of the two initial populations. The parameter values of the local structure of the decorated bilayers are assumed to be conserved in all particles, both before and after fusion.

\subsubsection{Resulting Calculated Scattering Curves}

Figure 6.5 (A) shows data $I(q)$ vs. $q$ from SVs in aqueous buffer (open circles, black), and a least-squares fit of a form factor model to the data (full line, red), as published in (15). The anisotropic model consists of a symmetric core profile (three concentric Gaussians) with attached Gaussian chains (solid red line, reduced $\chi^{2}=2.84$ ) on both sides of the bilayer profile. A sketch of a real space model corresponding to the least-squares fit of the form factor is shown in figure 4.1 (B). Pure PLs (dashed line, blue), assuming similar decorated bilayer structure, shape and size distribution as in the optimized SV model lead to a fairly similar scattering curve as compared to the SAXS data from SVs. However, since no larger contaminant particles are present, the scattering curve levels off faster towards small q values, and the shoulder at $q \approx 2 \times 10^{-1} \mathrm{~nm}^{-1}$, due to the washed out first form factor minimum, is more pronounced. A mixture of non-interacting SVs and PLs (full line, violet) leads to a very similar calculated scattering curve when compared to the SV SAXS data. However, the feature at $q \approx 2 \times 10^{-1} \mathrm{~nm}^{-1}$ is somewhat more pronounced. Fusion assays, where equal numbers of SVs and PLs are fused together (full line, green), as well as number ratios of 1:2 (SVs:PLs) lead to distinct scattering curves when compared to the SV SAXS data. The shoulder appearing at $q \approx 2 \times 10^{-1} \mathrm{~nm}^{-1}$ in the SV SAXS data moves significantly towards smaller q values, and gets increasingly pronounced.

Figure $6.5(B)$ shows the size polydispersity distribution functions $p(R)$ employed in the model calculations shown in (A). For all models involving SVs, a bimodal size polydispersity distribution function $p(R)$ was used, with one branch corresponding to cryo-EM data on the size distribution of the SVs, the PLs or the fusion products, and a second branch corresponding to larger membranous particles, modeled by a Gaussian distribution, as detailed in (15). Details on the SV form factor and the optimized parameter values obtained from the fit to the SV SAXS data which have been employed here, are given in $(15,17)$.

\subsubsection{Conclusions}

Calculated SAXS curves from the fusion product of SVs and PLs are found to be well distinct when compared to calculated SAXS patterns from a non-interacting mixture of SVs and PLs, or experimental SAXS data from SVs only. Further, it can be expected that docking (102), or aggregation, of the SVs and PLs would lead to distinct scattering patterns as compared to 
both the mixture of non-interacting SVs and PLs, and fused SV-PL. Thus, SAXS should be an ideal tool to discriminate between docking, hemi-fusion and fusion of SVs with PLs directly at a structural level. Other techniques routinely used to detect lipid mixing of fused or hemi-fused vesicles are typically based on fluorescence dequenching assays. While being faster and cheaper when compared to SAXS, these techniques do not provide direct structural information, and typically do not readily allow to distinguish between hemi-fusion and fusion of vesicles.

\subsection{Conclusions}

DLS is found to be a fast and reliable method which is capable to follow and quantify SNARE dependent fusion between small unilamellar proteo-liposomes and SVs under quasi-physiological conditions in a cell free fusion system. A Gedankenexperiment reveals that synchrotron based SAXS should be an ideal tool to elucidate the time resolved structural details on the nm scale of the fusion processes, including transient hemi-fusion structures in such ex vivo model fusion systems. 


\section{Chapter 7}

\section{Summary}

The aim of this thesis was

(i) to contribute to the understanding of the synaptic vesicle (SV) structure, and to the understanding of the processes of neuronal exocytosis and endocytosis, prominent examples of membrane trafficking in cells, (ii) to prepare and to characterize samples of SVs isolated from rat brain suited for investigation by small-angle x-ray scattering (SAXS), and to record solution SAXS data from SV dispersions under quasi-physiological conditions, (iii) to develop structural models for SVs, to calculate the corresponding scattering form factors, and to optimize and to falsify these form factors against experimentally determined scattering curves from SVs, (iv) to contribute to the understanding of fusion pathways by developing new approaches for structural investigation of cell free fusion systems.

In Chapter 2, an introduction to SVs, and to the isolation procedures of SVs from rat brain was given. Procedures have been presented and discussed to characterize and further enhance the purity of the SV ensembles, including cryogenic electron microscopy (cryo-EM), dynamic light scattering (DLS) and asymmetric-flow field-flow (AFFF) fractionation. Further, a preparation protocol of SV samples suited for solution SAXS experiments has been presented.

In Chapter 3, synchrotron based SAXS experiments have been presented, including the procedures employed for applying the instrumental corrections to the scattering data. Scattering curves from several individual SV samples have been recorded at two synchrotrons revealing high reproducibility of the data. No aggregation and no pronounced interaction potential was observed for the SVs of the investigated SV dispersions.

In Chapter 4, an introduction to kinematic scattering theory was given, and model independent as well as model based data analysis approaches were elucidated. Different isotropic and anisotropic structural models of SVs were developed, and the corresponding form factors were calculated. Further, a generalization of the form factor models was presented. Subsequently, the model form factors were optimized and falsified against SAXS data of SV dispersions under quasi-physiological conditions. All isotropic models were rejected, while anisotropic models 
were found to be in excellent agreement with a variety of experimental data of SVs, including SAXS data, cryo-EM data, biochemical data on the stoichiometry of the SV, and structural data of individual proteins on the SV. SAXS data from model liposomes and protease treated SVs were analyzed to investigate and assess how the model reflects distinct structural changes of the samples.

In Chapter 5, an interpretation and discussion of the different SV models were given, addressing in particular the size polydispersity distribution and the bilayer structure. Proteins were found to be clustered, forming micro-domains on the SV. Possible model dependencies and ambiguities were discussed. Model entropy considerations were presented revealing that entropic contributions originating from protein cluster formation and disintegration may play important roles to provide free energy needed for driving fusion of the SV with the plasma membrane.

In Chapter 6, a cell free fusion system was presented consisting of SV isolated from rat brain, and proteo-liposomes with reconstituted t-SNARE proteins. Dynamic light scattering was used to determine the size distribution of these particles, and to quantify the size increase associated with fusion of SVs with proteo-liposomes. A Gedankenexperiment with calculated scattering curves reveals that small-angle x-ray scattering is likely to resolve the fusion pathway of SVs and proteo-liposomes directly on a structural level.

In summary, we conclude that direct modeling of solution small-angle x-ray scattering data, considered in combination with data obtained by other analytical techniques such as cryogenic electron microscopy, dynamic light scattering, biochemical analysis and protein crystallography, reveals the heterogeneous structure of synaptic vesicles isolated from rat brain under quasi-physiological conditions. The overall low resolution structure of the entire synaptic vesicle has been obtained, elucidating details on the density profile of the membrane, including contributions from the lipids and proteins, as well as addressing the average conformation and overall lateral organization of proteins in micro-domains on the average synaptic vesicle under quasi-physiological conditions.

The organization of the proteins in micro-domains on the SV suggests that entropic contributions to the free energy due to protein cluster formation and disintegration may significantly influence the processes of membrane merger and budding in neuronal exocytosis and endocytosis.

The structural information of the SV may contribute to describing and understanding the processes of membrane fusion, retrieval and recycling, related to neuronal exocytosis, and to membrane trafficking in eukaryotic cells in general.

Further, a Gedankenexperiment reveals that it seems likely that SAXS is suited to study fusion pathways of cell free fusion systems consisting of synaptic vesicles isolated from rat brain and proteo-liposomes with reconstituted t-SNARE proteins directly on a structural level.

A particular advantage of SAXS is the more physiological state of the sample when compared 
to other techniques, e. g. electron microscopy, typically requiring invasive sample preparation steps such as fixation or staining which are prone to introduce artifacts into the analysis. In contrast, SAXS is compatible with a large spectrum of physical and chemical parameters of the sample such as temperature, pressure, or ion concentrations. The changes induced in the sample by the variation of parameters can be studied directly on a structural level in real time with up to ms time resolution. A large number of samples can be collected and compared due to the small sample quantities needed for high brilliance synchrotron SAXS (on the order of $10^{-4}$ to $10^{-3} \mu \mathrm{l}$ ). Other techniques like in-house SAXS or transmission electron microscopy would consume too much time and material, effectively inhibiting the investigation of similar sample series. Our present SAXS data on synaptic vesicles employs scattering intensities up to $q=2.7 \mathrm{~nm}^{-1}$. The $q$-range could be further extended by increasing the final vesicle concentration through modifications in the purification protocol. A theoretical concentration increase of 100-fold should extend the exploitable $q$-range by a factor of three, and increase the obtainable resolution to about $0.8 \mathrm{~nm}$. However, at high vesicle concentrations, effects of inter-particle interactions to the scattering data would need to be explicitly modeled, for example by employing a corresponding structure factor in the data analysis. Such studies should shed light on the nature of the interaction between synaptic vesicles, including the role of the proteins on the surface of the vesicles. Further, results from studies of vesicles isolated from wild-type animals could be augmented with studies of vesicles from so-called knock-in and knock-out animals, to genetically manipulate essential components of the system (S. Ahmed, M. Holt, D. Riedel and R. Jahn, accepted). Furthermore, SAXS could be employed to determine time-resolved structural changes resulting from membrane interaction and membrane fusion, in combination with microfluidic devices and systems combining top-down and bottom-up approaches.

With the advent of x-ray lasers delivering ultra short and extremely intensive $\mathrm{x}$-ray pulses, time resolved scattering experiments at individual organelles and fusion intermediates might eventually become possible. In conjunction with computer simulations of coarse-grained models and atomistic models of membrane pore formation and fusion in the presence of proteins, such experiments would undoubtedly shed light on the biophysical principles of membrane merger. 


\section{Bibliography}

[1] Combes, F., and A. Mazure, 2002. Galaxies and cosmology. Springer Verlag.

[2] Nicholls, J., R. Martin, B. Wallace, and P. Fuchs, 2001. From neuron to brain (4th). New York: Sinauer Inc. Publishers.

[3] Lodish, H., A. Berk, L. Zipursky, P. Matsudaira, D. Baltimore, and J. Darnell, 2000. Molecular Cell Biology. W. H. Freeman.

[4] Siegel, G., R. Albers, S. Brady, and D. Price, 2006. Basic neurochemistry: molecular, cellular, and medical aspects. Academic Press.

[5] Jahn, R., 2008. Some classic papers in the field of membrane fusion-a personal view. Nature structural \& molecular biology 15:655-657.

[6] Wickner, W., and R. Schekman, 2008. Membrane fusion. Nature structural 8 molecular biology 15:658-664.

[7] Jahn, R., and T. Südhof, 1999. Membrane fusion and exocytosis. Annual review of biochemistry 68:863-911.

[8] Israelachvili, J., 1991. Intermolecular and surface forces. Academic press London.

[9] Gelbart, W., W. Gelbart, A. Ben-Shaul, and D. Roux, 1994. Micelles, membranes, microemulsions, and monolayers. Springer.

[10] Jahn, R., T. Lang, and T. Südhof, 2003. Membrane fusion. Cell 112:519-533.

[11] Jahn, R., and T. Sudhof, 1994. Synaptic vesicles and exocytosis. Annual review of neuroscience 17:219-246.

[12] Lin, R., and R. Scheller, 2000. Mechanisms of synaptic vesicle exocytosis. Annual Review of Cell and Developmental Biology 16:19-49.

[13] Jahn, R., and R. Scheller, 2006. SNAREs-engines for membrane fusion. Nature Reviews Molecular Cell Biology 7:631-643. 
[14] Takamori, S., M. Holt, K. Stenius, E. A. Lemke, M. Grønborg, D. Riedel, H. Urlaub, S. Schenck, B. Brügger, P. Ringler, S. A. Müller, B. Rammner, F. Gräter, J. S. Hub, B. L. De Groot, G. Mieskes, Y. Moriyama, J. Klingauf, H. Grubmüller, J. Heuser, F. Wieland, and R. Jahn, 2006. Molecular Anatomy of a Trafficking Organelle. Cell 127:831 - 846.

[15] Castorph, S., D. Riedel, L. Arleth, M. Sztucki, R. Jahn, M. Holt, and T. Salditt, 2010. Structure Parameters of Synaptic Vesicles Quantified by Small-Angle X-ray Scattering. Biophys. J. 98:1200-1208.

[16] Castorph, S., S. Schwarz Henriques, M. Holt, D. Riedel, R. Jahn, and T. Salditt. Synaptic Vesicles Studied by Dynamic Light Scattering. In preparation .

[17] Castorph, S., L. Arleth, M. Sztucki, U. Vainio, S. K. Ghosh, M. Holt, R. Jahn, and T. Salditt, 2010. Synaptic Vesicles Studied by SAXS: Derivation and Validation of a Model Form Factor. J. Phys.: Conf. Ser., 247:012015 (17pp).

[18] Ghosh, S. K., S. Castorph, O. Konovalov, R. Jahn, M. Holt, and T. Salditt, 2010. In vitro study of interaction of synaptic vesicles with lipid membranes. New J. Phys., 12:105004 (24pp).

[19] Südhof, T. C., 2004. The synaptic vesicle cycle. Annu. Rev. Neurosci. 27:509 - 547.

[20] Baumert, M., P. R. Maycox, F. Navone, P. De Camilli, and R. Jahn, 1989. Synaptobrevin: an integral membrane protein of 18,000 daltons present in small synaptic vesicles of rat brain. EMBO J. 8:379-384.

[21] Nagy, A., R. R. Baker, S. J. Morris, and V. P. Whittaker, 1976. The preparation and characterization of synaptic vesicles of high purity. Brain Res. 109:285-309.

[22] Betzig, E., G. Patterson, R. Sougrat, O. Lindwasser, S. Olenych, J. Bonifacino, M. Davidson, J. Lippincott-Schwartz, and H. Hess, 2006. Imaging intracellular fluorescent proteins at nanometer resolution. Science 313:1642.

[23] Hell, S. W., and J. Wichmann, 1994. Breaking the diffraction resolution limit by stimulated emission: stimulated-emission-depletion fluorescence microscopy. Opt. Lett 19:780782.

[24] Giewekemeyer, K., H. Neubauer, S. Kalbfleisch, S. Krüger, and T. Salditt, 2010. Holographic and diffractive x-ray imaging using waveguides as quasi-point sources. New Journal of Physics 12:035008. 
[25] Alber, F., F. Förster, D. Korkin, M. Topf, and A. Sali, 2008. Integrating diverse data for structure determination of macromolecular assemblies. Annu. Rev. Biochem. 77:443 -477 .

[26] Sano, Y., H. Inoue, K. Kajiwara, Y. Hiragi, and S. Isoda, 1997. Structural analysis of Aprotein of cucumber green mottle mosaic virus and tobacco mosaic virus by synchrotron small-angle X-ray scattering. J. Protein Chem. 16:151-159.

[27] Maycox, P. R., T. Deckwerth, J. W. Hell, and R. Jahn, 1988. Glutamate uptake by brain synaptic vesicles. Energy dependence of transport and functional reconstitution in proteoliposomes. J. Biol. Chem. 263:15423-15428.

[28] Schwarz Gonçalves Henriques, S. M., 2008. Laser Light Scattering at Synaptic Vesicles. Diploma thesis, Georg-August-Universität Göttingen.

[29] Berne, B. J., and R. Pecora, 2000. Dynamic Light Scattering. Dover Publications Inc.

[30] Lindner, P., and T. Zemb, editors, 2002. Neutrons, X-rays and Light: Scattering Methods Applied to Soft Condensed Matter. North-Holland, Elsevier.

[31] Brown, W., 1993. Dynamic light scattering: the method and some applications. Oxford University Press, USA.

[32] Baker, C., L. Fox, D. Mayers, and K. Wright, 1964. Numerical solution of Fredholm integral equations of first kind. The Computer Journal 7:141.

[33] McWhirter, J., and E. Pike, 1978. On the numerical inversion of the Laplace transform and similar Fredholm integral equations of the first kind. Journal of Physics A: Mathematical and General 11:1729-1745.

[34] Honerkamp, J., and J. Weese, 1990. Tikhonovs regularization method for ill-posed problems. Continuum Mechanics and Thermodynamics 2:17-30.

[35] Provencher, S., 1982. A constrained regularization method for inverting data represented by linear algebraic or integral equations. Computer Physics Communications 27:213-227.

[36] Provencher, S., 1982. CONTIN: a general purpose constrained regularization program for inverting noisy linear algebraic and integral equations. Comput. Phys. Commun 27:229242.

[37] Schatzel, K., M. Drewel, and S. Stimac, 1988. Photon correlation measurements at large lag times: improving statistical accuracy. Journal of Modern Optics 35:711-718. 
[38] Schatzel, K., 1990. Noise on photon correlation data. I. Autocorrelation functions. Quantum Optics: Journal of the European Optical Society Part B 2:287-305.

[39] Guiddings, J., 1993. Field-flow fractionation: analysis of macromolecular, colloidal, and particulate materials. Science 260.

[40] Wyatt, P., 1998. Submicrometer particle sizing by multiangle light scattering following fractionation. Journal of colloid and interface science 197:9-20.

[41] Korgel, B., J. van Zanten, and H. Monbouquette, 1998. Vesicle size distributions measured by flow field-flow fractionation coupled with multiangle light scattering. Biophysical journal 74:3264-3272.

[42] Mandel, L., 1963. Fluctuations of Light Beams, volume II. North-Holland Publishing Company.

[43] Hell, J. W., R. P. Maycox, H. Stadler, and R. Jahn, 1988. Uptake of GABA by rat brain synaptic vesicles isolated by a new procedure. EMBO J., 7:3023-3029.

[44] Perin, M., N. Brose, R. Jahn, and T. Südhof, 1991. Domain structure of synaptotagmin (p65). Journal of Biological Chemistry 266:623.

[45] Narayanan, T., O. Diat, and P. Boesecke, 2001. SAXS and USAXS on the high brilliance beamline at the ESRF. Nucl. Instrum. Methods Phys. Res., Sect. A 467:1005-1009.

[46] Lotze, G., 2008. Röntgenanalyse der Amyloid-Aggregation am Modellprotein Insulin. Diploma thesis, Georg-August-Universität Göttingen.

[47] Haubold, H.-G., K. Gruenhagen, M. Wagener, H. Jungbluth, a. A. Heer, H., H. Rongen, G. Brandenberg, R. Moeller, J. Matzerath, P. Hiller, and H. Halling, 1989. JUSIFA - A new user dedicated ASAXS beamline for materials science. Rev. Sci. Instrum. 60:19431946.

[48] Krolzig, A., G. Materlik, M. Swars, and J. Zegenhagen, 1984. A feedback control system for synchrotron radiation double crystal instruments. Nuclear Instruments and Methods in Physics Research 219:430-434.

[49] Guinier, A., and G. Fournet, 1955. Small Angle Scattering of X-rays. Wiley Interscience, New York.

[50] Alberts, B., A. Johnson, J. Lewis, M. Raff, K. Roberts, and P. Walter, 2008. Molecular biology of the cell. Gerland Science, 5th edition. 
[51] Howells, E., T. Beetz, H. N. Chapman, C. Cui, J. M. Holton, C. J. Jacobsen, J. Kirz, E. Lima, S. Marchesini, H. Miao, D. Sayre, D. A. Shapiro, J. C. H. Spence, and D. Starodub, 2009. An assessment of the resolution limitation due to radiation-damage in X-ray diffraction microscopy. J. Electron Spectrosc. Relat. Phenom. 170:4-12.

[52] Glatter, O., 1977. A new method for the evaluation of small-angle scattering data. J. Appl. Crystallogr. 10:415-421.

[53] Glatter, O., 1980. Determination of particle-size distribution functions from small-angle scattering data by means of the indirect transformation method. J. Appl. Crystallogr. 13:7-11.

[54] Glatter, O., 1980. Evaluation of small-angle scattering data from lamellar and cylindrical particles by the indirect transformation method. J. Appl. Crystallogr. 13:577-584.

[55] Greville, T., 1969. Introduction to spline functions. Theory and applications of spline functions $1-35$.

[56] Schelten, J., and F. Hossfeld, 1971. Application of spline functions to the correction of resolution errors in small-angle scattering. J. Appl. Crystallogr. 4:210-223.

[57] Glatter, O., 1981. Convolution square root of band-limited symmetrical functions and its application to small-angle scattering data. J. Appl. Crystallogr. 14:101-108.

[58] Glatter, O., and B. Hainisch, 1984. Improvements in real-space deconvolution of smallangle scattering data. J. Appl. Crystallogr. 17:435-441.

[59] Mittelbach, R., and O. Glatter, 1998. Direct structure analysis of small-angle scattering data from polydisperse colloidal particles. J. Appl. Crystallogr. 31:600-608.

[60] Svergun, D., 1999. Restoring low resolution structure of biological macromolecules from solution scattering using simulated annealing. Biophys. J. 76:2879-2886.

[61] Svergun, D., 1994. Solution scattering from biopolymers: advanced contrast-variation data analysis. Acta Crystallographica Section A: Foundations of Crystallography 50:391402.

[62] Petoukhov, M., and D. Svergun, 2005. Global rigid body modeling of macromolecular complexes against small-angle scattering data. Biophys. J. 89:1237-1250.

[63] Petoukhov, M., and D. Svergun, 2007. Analysis of X-ray and neutron scattering from biomacromolecular solutions. Current opinion in structural biology 17:562-571. 
[64] Pedersen, J., 1997. Analysis of small-angle scattering data from colloids and polymer solutions: modeling and least-squares fitting. Advances in colloid and interface science 70:171-210.

[65] Bouwstra, J. A., G. S. Gorris, W. Bras, and H. Talsma, 1993. Small angle X-ray scattering: possibilities and limitations in characterization of vesicles. Chemistry and Physics of Lipids 64:83 - 98 .

[66] Pabst, G., M. Rappolt, H. Amenitsch, and P. Laggner, 2000. Structural information from multilamellar liposomes at full hydration: Full q-range fitting with high quality x-ray data. Phys. Rev. E 62:4000-4009.

[67] Pabst, G., R. Koschuch, B. Pozo-Navas, M. Rappolt, K. Lohner, and P. Laggner, 2003. Structural analysis of weakly ordered membrane stacks. J. Appl. Crystallogr. 36:13781388 .

[68] Gradzielski, M., D. Langevin, L. Magid, and R. Strey, 1995. Small-Angle Neutron Scattering from Diffuse Interfaces. J. Phys. Chem. 99:13232 - 13238.

[69] Debye, P., 1947. Molecular weights of proteins from light scattering. J. Phys. Colloid Chem. $51: 18-32$.

[70] Pedersen, J. S., and M. C. Gerstenberg, 1996. Scattering Form Factor of Block Copolymer Micelles. Marcromolecules 29:1363 - 1365.

[71] Pedersen, J. S., 2000. Form factors of block copolymer micelles with spherical, ellipsoidal and cylindrical cores. J. Appl. Crystallogr. 33:637 - 640.

[72] Pedersen, J. S., 2001. Structure factors effects in small-angle scattering from block copolymer micelles and star polymers. J. Chem. Phys. 114:2839- 2846.

[73] Arleth, L., and C. Vermehren, 2010. An analytical model for the small-angle scattering of polyethylene glycol-modified liposomes. J. Appl. Crystallogr. 43:1084-1091.

[74] Doi, M., and S. Edwards, 1986. The Theory of Polymer Dynamics. Oxford University Press.

[75] Hammouda, B., 1992. Structure Factor for Starburst Dentrimers. Journal of Polymer Science B 30:1387 - 1390.

[76] Debye, P., 1915. Zerstreuung von Roentgenstrahlung. Ann. Phys. 46:809-823.

[77] Debye, P., and A. Bueche, 1949. Scattering by an inhomogeneous solid. Journal of Applied Physics 20:518-525. 
[78] Glatter, O., 1979. The interpretation of real-space information from small-angle scattering experiments. J. Appl. Cryst. 12:166-175.

[79] Gille, W., 2007. Small-angle scattering analysis of the spherical half-shell. J. Appl. Cryst. 40:302-304.

[80] Watson, G., 1995. A treatise on the theory of Bessel functions. Cambridge mathematical library. Cambridge University Press.

[81] Abramowitz, M., and I. Stegun, 1972. Handbook of mathematical functions with formulas, graphs, and mathematical tables. Dover books on mathematics. Dover New York.

[82] Bates, D., and D. Watts, 1988. Nonlinear regression analysis and its applications. John Wiley \& Sons New York.

[83] Seber, G., and C. Wild, 2003. Nonlinear Regression. Wiley.

[84] Kucerka, N., J. Gallová, D. Uhrıková, P. Balgavỳ, M. Bulacu, S. Marrink, and J. Katsaras, 2009. Areas of Monounsaturated Diacylphosphatidylcholines. Biophys. J. 97:1926-1932.

[85] Qu, L., Y. Akbergenova, Y. Hu, and T. Schikorski, 2009. Synapse-to-synapse variation in mean synaptic vesicle size and its relationship with synaptic morphology and function. J. Comp. Neurol. 514:343-352.

[86] Burger, P. M., E. Mehl, P. L. Cameron, P. R. Maycox, M. Baumert, F. Lottspeich, P. De Camilli, and R. Jahn, 1989. Synaptic vesicles immunoisolated from rat cerebral cortex contain high levels of glutamate. Neuron 3:715 - 720.

[87] Zhang, B., Y. H. Koh, R. B. Beckstead, V. Budnik, B. Ganetzky, and H. J. Bellen, 1998. Synaptic vesicle size and number are regulated by a clathrin adaptor protein required for endocytosis. Neuron 21:1465 - 1475 .

[88] Loerke, D., M. Mettlen, D. Yarar, K. Jaqaman, H. Jaqaman, G. Danuser, and S. L. Schmid, 2009. Cargo and dynamin regulate clathrin-coated pit maturation. PLoS Biol. 7:e57.

[89] Fernandez-Alfonso, T., R. Kwan, and T. A. Ryan, 2006. Synaptic vesicles interchange their membrane proteins with a large surface reservoir during recycling. Neuron 51:179186.

[90] Edwards, R. H., 2007. The neurotransmitter cycle and quantal size. Neuron 55:835 858. 
[91] Zenisek, D., J. A. Steyer, and W. Almers, 2000. Transport, capture and exocytosis of single synaptic vesicles at active zones. Nature 406:849-854.

[92] Jia, J. Y., S. Lamer, M. Schümann, M. R. Schmidt, E. Krause, and V. Haucke, 2006. Quantitative proteomics analysis of detergent-resistent membranes from chemical synapses: evidence for cholesterol as spatial organizer of synaptic vesicle cycling. Mol. Cell Proteomics 20:60 - 71.

[93] Willig, K., S. Rizzoli, V. Westphal, R. Jahn, and S. Hell, 2006. STED microscopy reveals that synaptotagmin remains clustered after synaptic vesicle exocytosis. Nature 440:935939.

[94] Nagle, J., and S. Tristram-Nagle, 2000. Structure of lipid bilayers. . Biochim. Biphys. Acta 1469:159-195.

[95] Gruenberg, J., and K. Howell, 1989. Membrane traffic in endocytosis: insights from cell-free assays. Annual review of cell biology 5:453-481.

[96] Holt, M., D. Riedel, A. Stein, C. Schuette, and R. Jahn, 2008. Synaptic vesicles are constitutively active fusion machines that function independently of Ca2+. Current Biology 18:715-722.

[97] Smith, H., M. Jablin, A. Vidyasagar, J. Saiz, E. Watkins, R. Toomey, A. Hurd, and J. Majewski, 2009. Model Lipid Membranes on a Tunable Polymer Cushion. Phys. Rev. Lett. 102:228102.

[98] Hosoi, N., M. Holt, and T. Sakaba, 2009. Calcium dependence of exo-and endocytotic coupling at a glutamatergic synapse. Neuron 63:216-229.

[99] Schuette, C., K. Hatsuzawa, M. Margittai, A. Stein, D. Riedel, P. Küster, M. König, C. Seidel, and R. Jahn, 2004. Determinants of liposome fusion mediated by synaptic SNARE proteins. Proc. Natl. Acad. Sci. U S A 101:2858.

[100] McMahon, H., Y. Ushkaryov, L. Edelmann, E. Link, T. Binz, H. Niemann, R. Jahn, and T. Südhof, 1993. Cellubrevin is a ubiquitous tetanus-toxin substrate homologous to a putative synaptic vesicle fusion protein. Nature 364:346-349.

[101] Zilly, F., J. Sorensen, R. Jahn, and T. Lang, 2006. Munc18-bound syntaxin readily forms SNARE complexes with synaptobrevin in native plasma membranes. PLoS Biol 4:e330.

[102] Cypionka, A., A. Stein, J. Hernandez, H. Hippchen, R. Jahn, and P. Walla, 2009. Discrimination between docking and fusion of liposomes reconstituted with neuronal SNAREproteins using FCS. Proc. Natl. Acad. Sci. U S A 106:18575. 
Appendices 



\section{Appendix A}

\section{MATLAB Source Code}

This section presents the essential procedures of the computer programs developed within the scope of this thesis. The script files are written in the MATLAB programming language (Version 7.5.0.342 (R2007b), The MathWorks Inc.). In MATLAB, comments are denoted by the per cent sign, and three points in a row indicate that the respective command continues on the next line.

\section{A.1 Direct Modeling Dynamic Light Scattering Data}

The direct modeling approach of dynamic light scattering (DLS) data presented in section 2.6 was implemented in MATLAB. The optimization was carried out by employing the lsqnonlin routine of the Optimization Toolbox (Version 3.1 (R2006b), The MathWorks Inc.), dedicated to solve nonlinear least-squares problems. Since the complete code is too long for this thesis, only the procedure ObjFunVec_DLS.m carrying out the calculation of the object function (cost function to be minimized), including the form factor and the size polydispersity calculation, are given here.

\section{A.1.1 ObjFunVec_DLS.m}

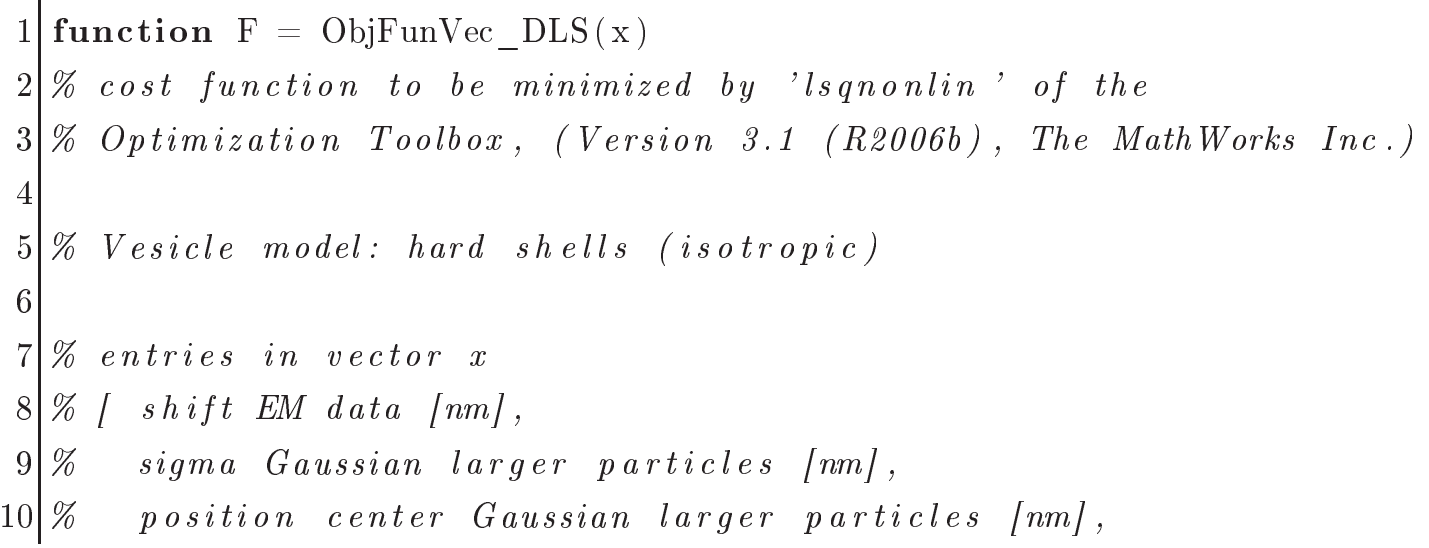









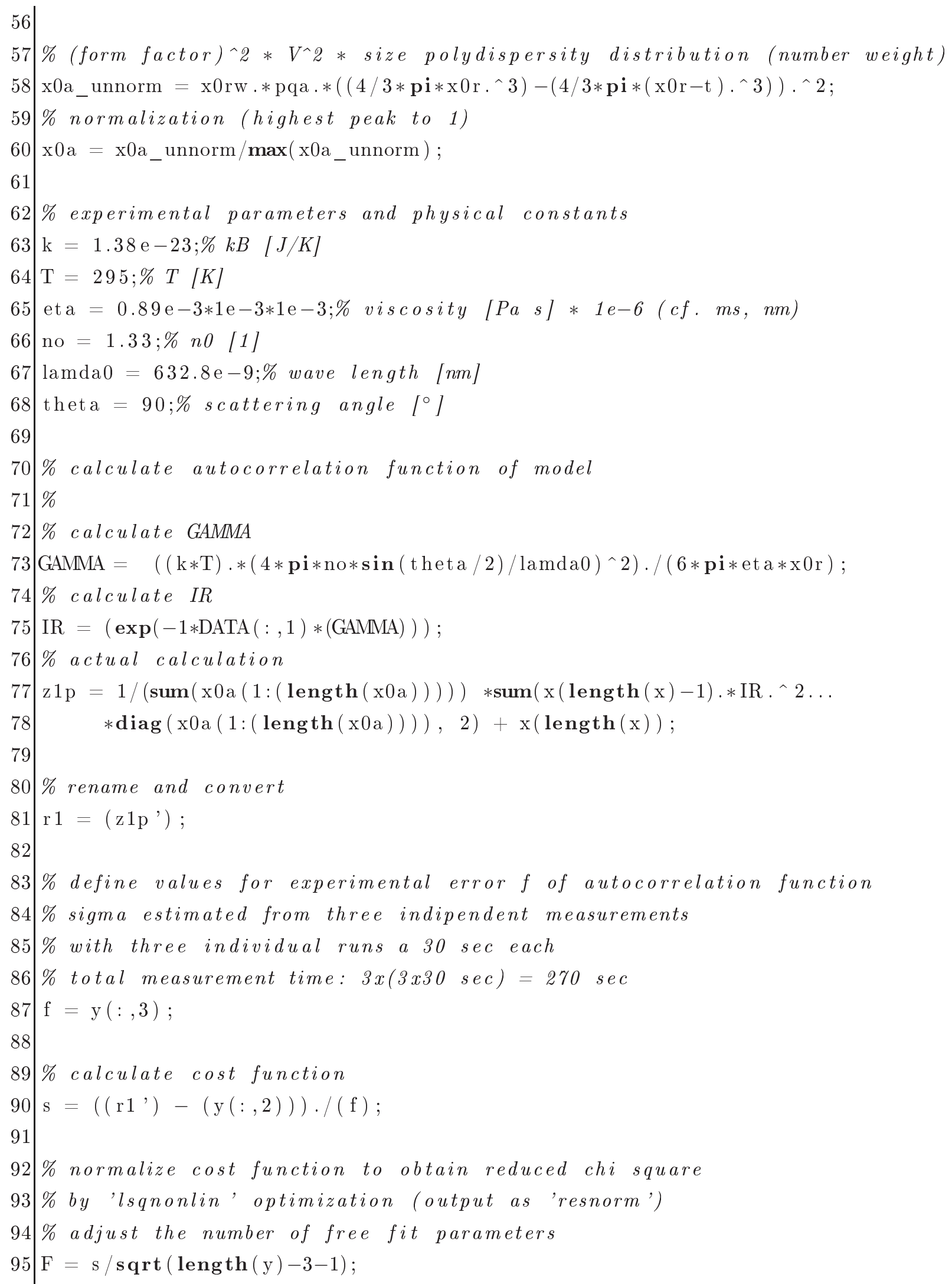




\section{A.2 Direct Modeling Small-Angle X-Ray Scattering Data}

The model optimization and falsification against small-angle x-ray scattering data presented in section 4.6 was implemented in MATLAB. The main procedure calls a function which loads the background corrected 1D SAXS data from dat files into MATLAB, combines SAXS data recorded at different detector distances, and subtracts the scattering curves recorded from a capillary filled with plain aqueous buffer from the scattering curve of SVs. The final SAXS curve for fitting may be re-binned, and/or multiplied by $q^{2}$ by uncommenting the corresponding command lines. The optimization was carried out by employing the lsqnonlin routine of the Optimization Toolbox (Version 3.1 (R2006b), The MathWorks Inc.), dedicated to solve nonlinear least-squares problems. The lsqnonlin routine calls the function ObjFunVec_SAXS.m containing the code for the calculation of the object function. Plotting is handled separately by a dedicated routine. Subsequently, approximate marginal confidence bounds (95\%) for the parameter values estimated by the nonlinear regression of the models to the SAXS data were calculated using the nlparci routine of MATLAB Statistics Toolbox (Version 7.5.0.342 (R2007b), The MathWorks Inc.), and the optimized model parameters were calibrated to an absolute scale.

Since the complete code is too long for this thesis, only the procedure ObjFunVec_SAXS.m carrying out the calculation of the vectorized object function (cost function to be minimized), including the calculation of the scattering signal of the bimodal size distributed model particles employing the anisotropic form factor with Gaussian chains given in section 4.5, are given here.

\section{A.2.1 ObjFunVec SAXS.m}

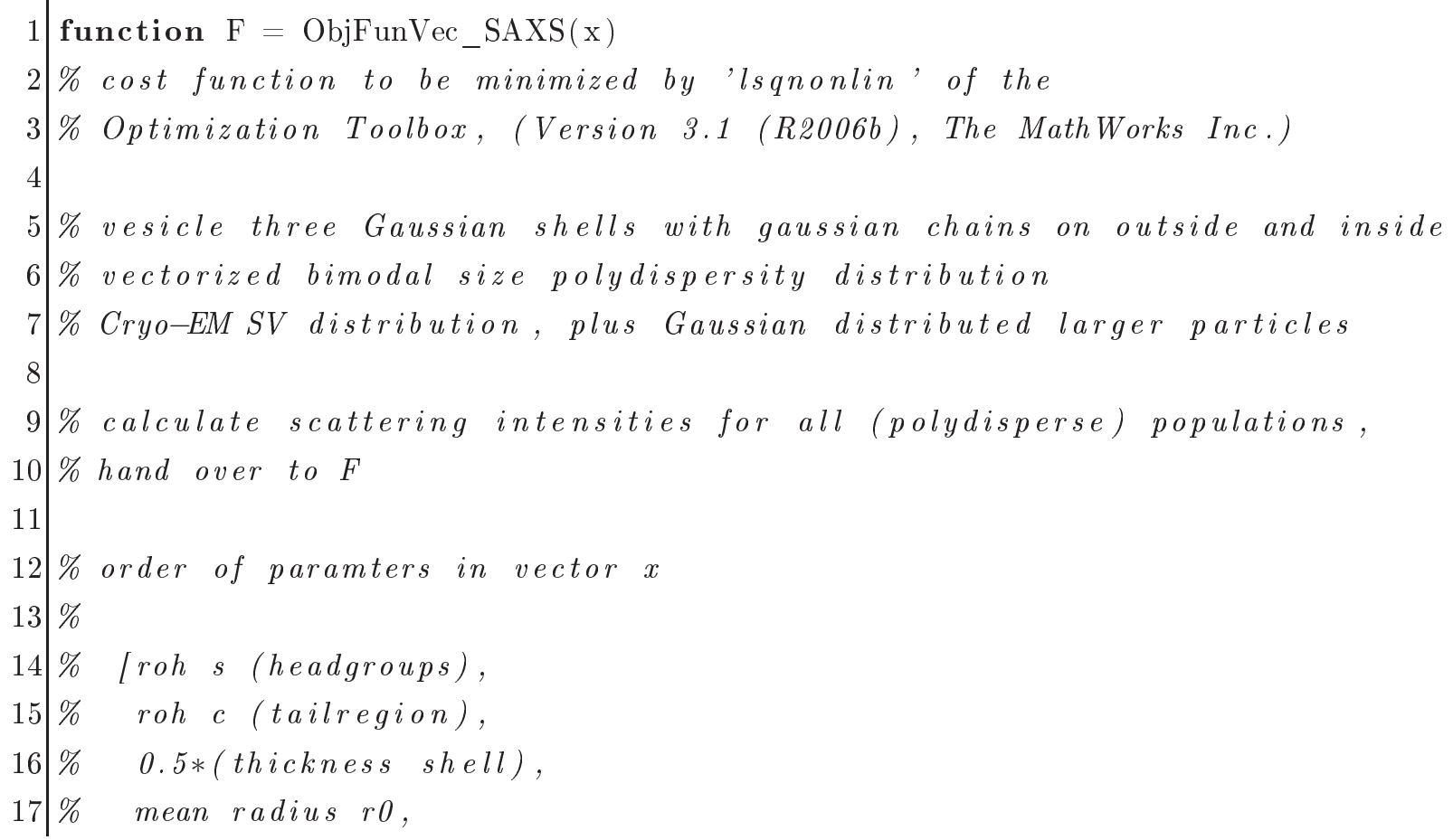




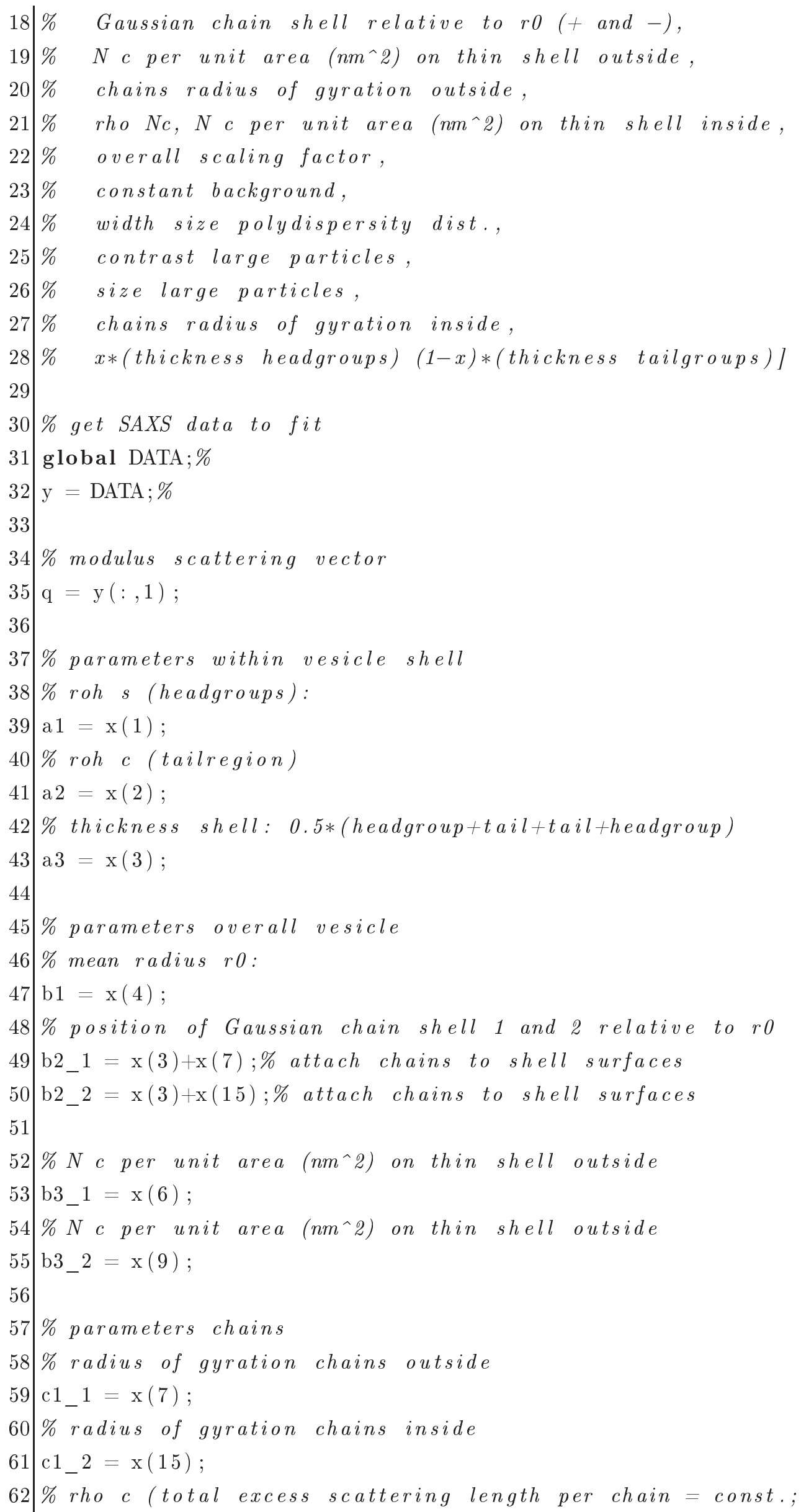




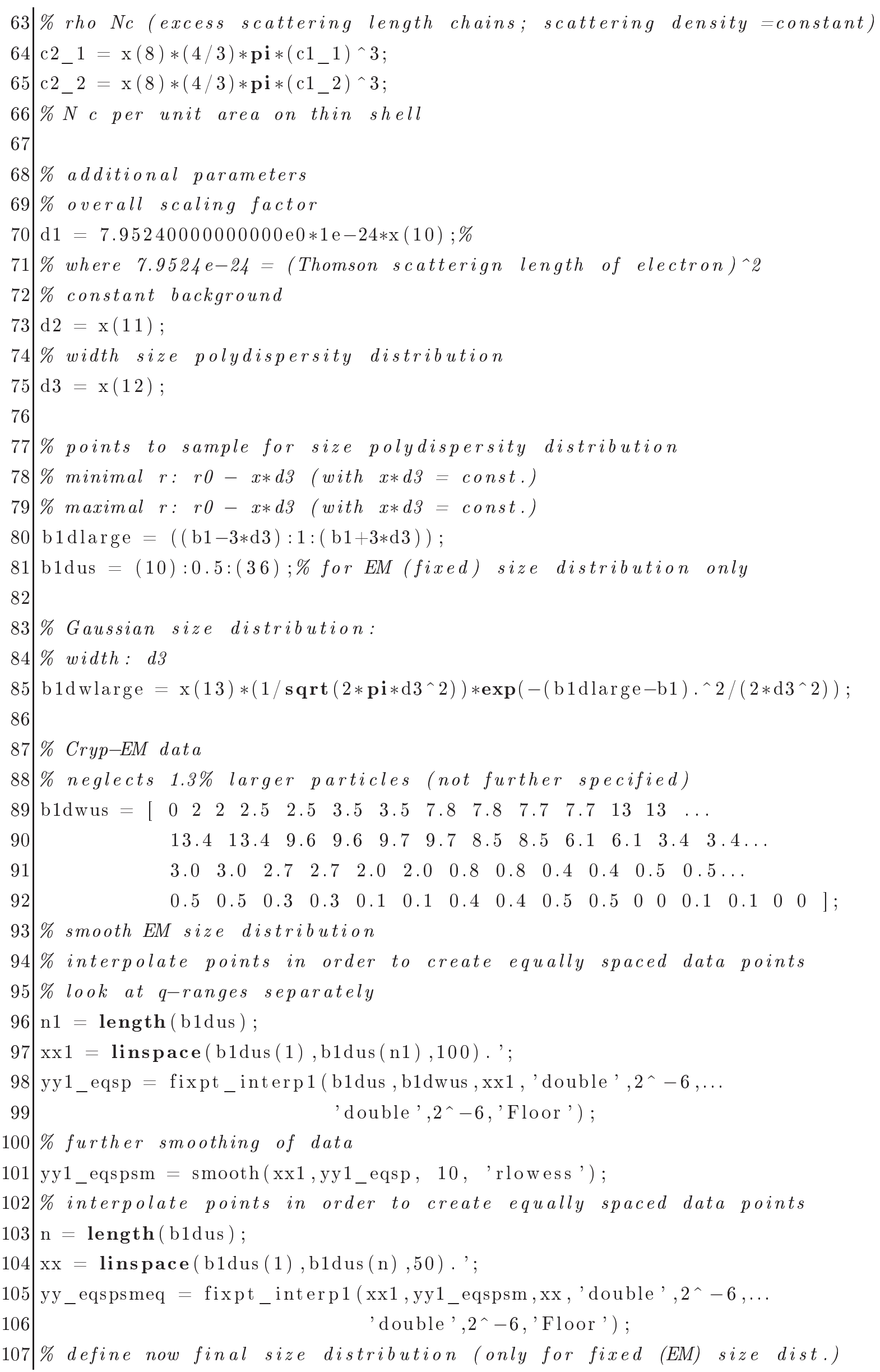




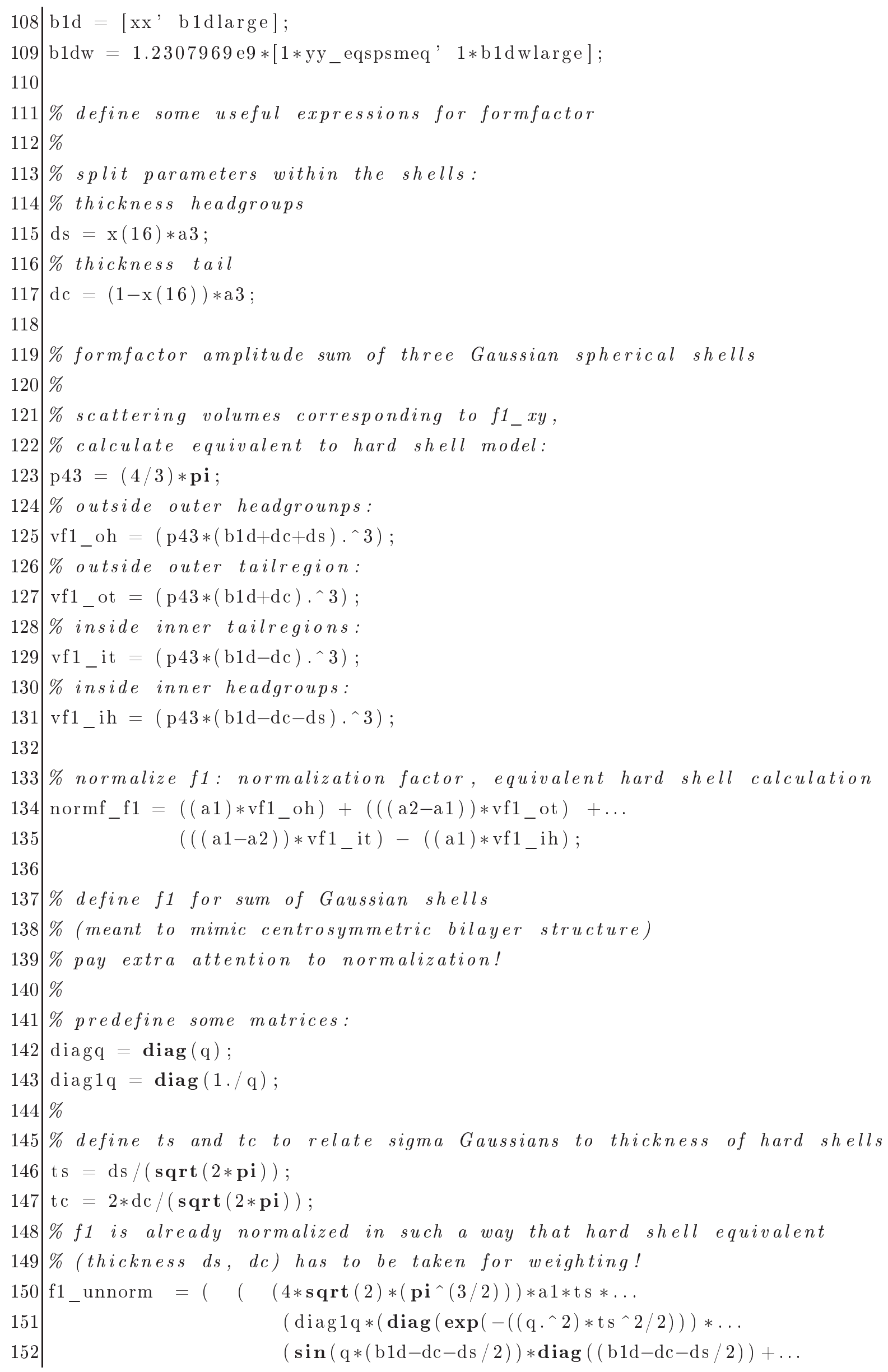




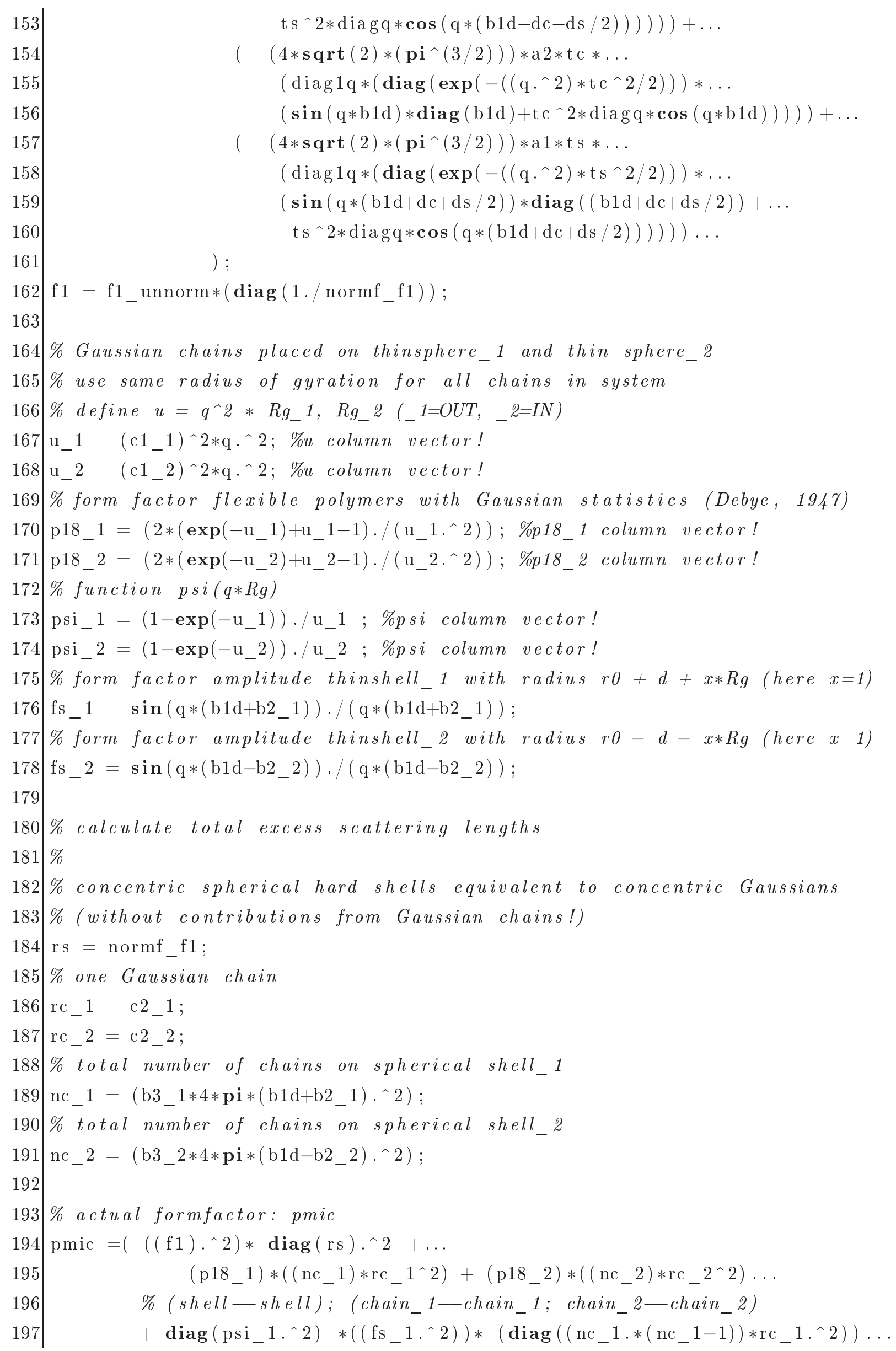




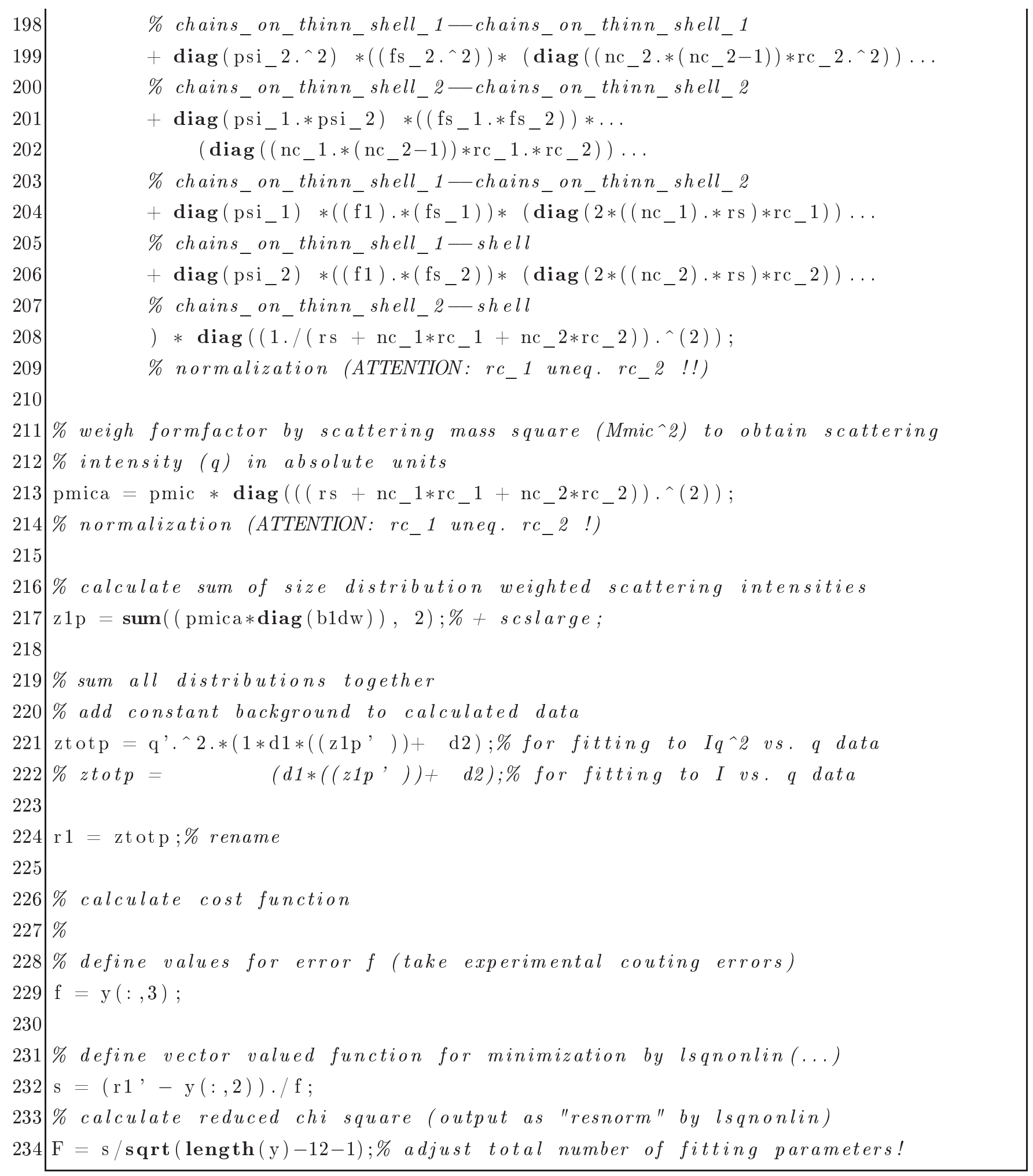

\section{A.3 Model Entropy Calculations}

The model entropy calculation presented in section 5.2 was implemented in MATLAB. The main procedure is main_entropy.m. The function ln_fact.m calculates the logarithm of the faculty for a natural number. For numbers larger than 20, the Stirling approximation is used. 


\section{A.3.1 main entropy.m}

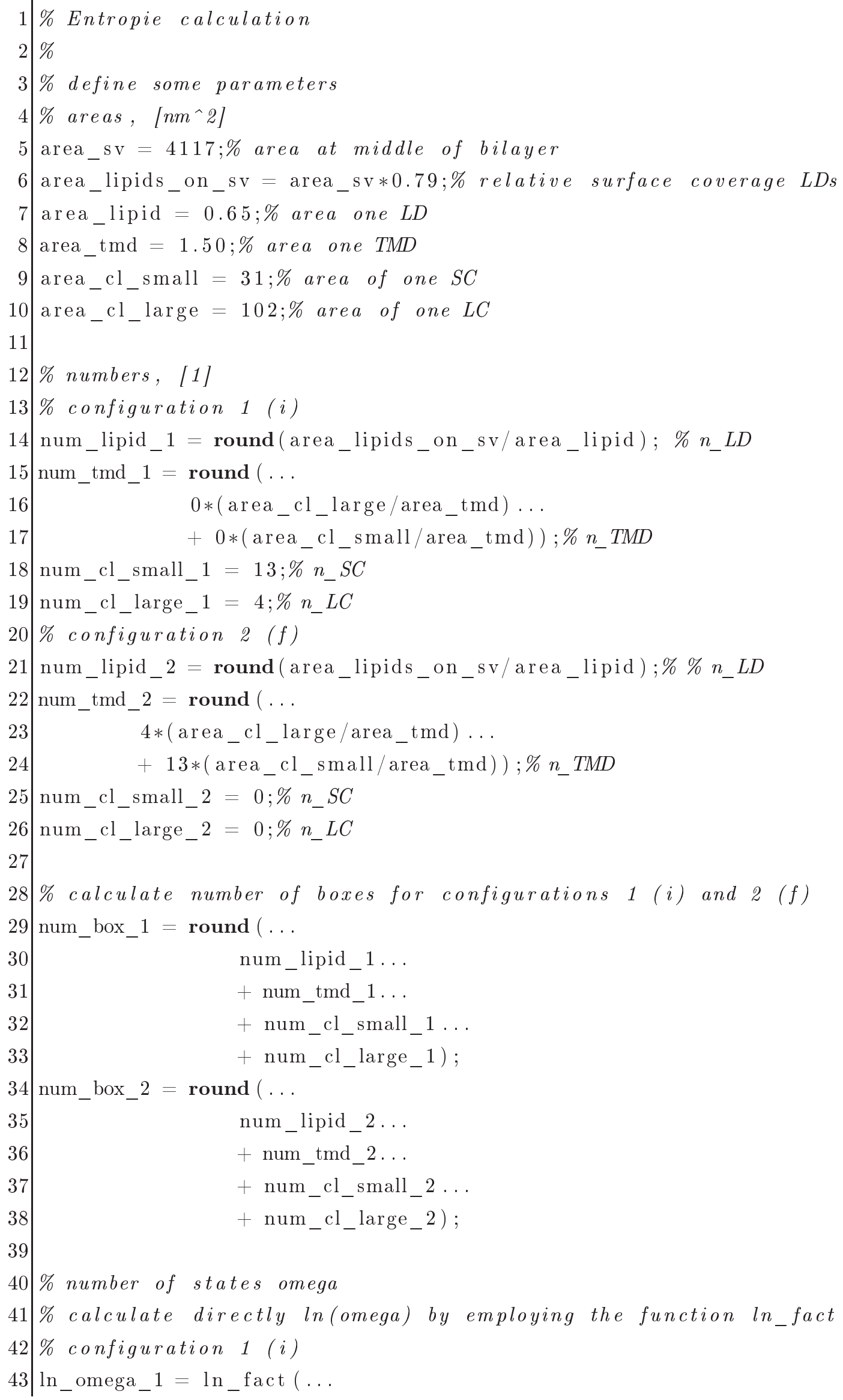




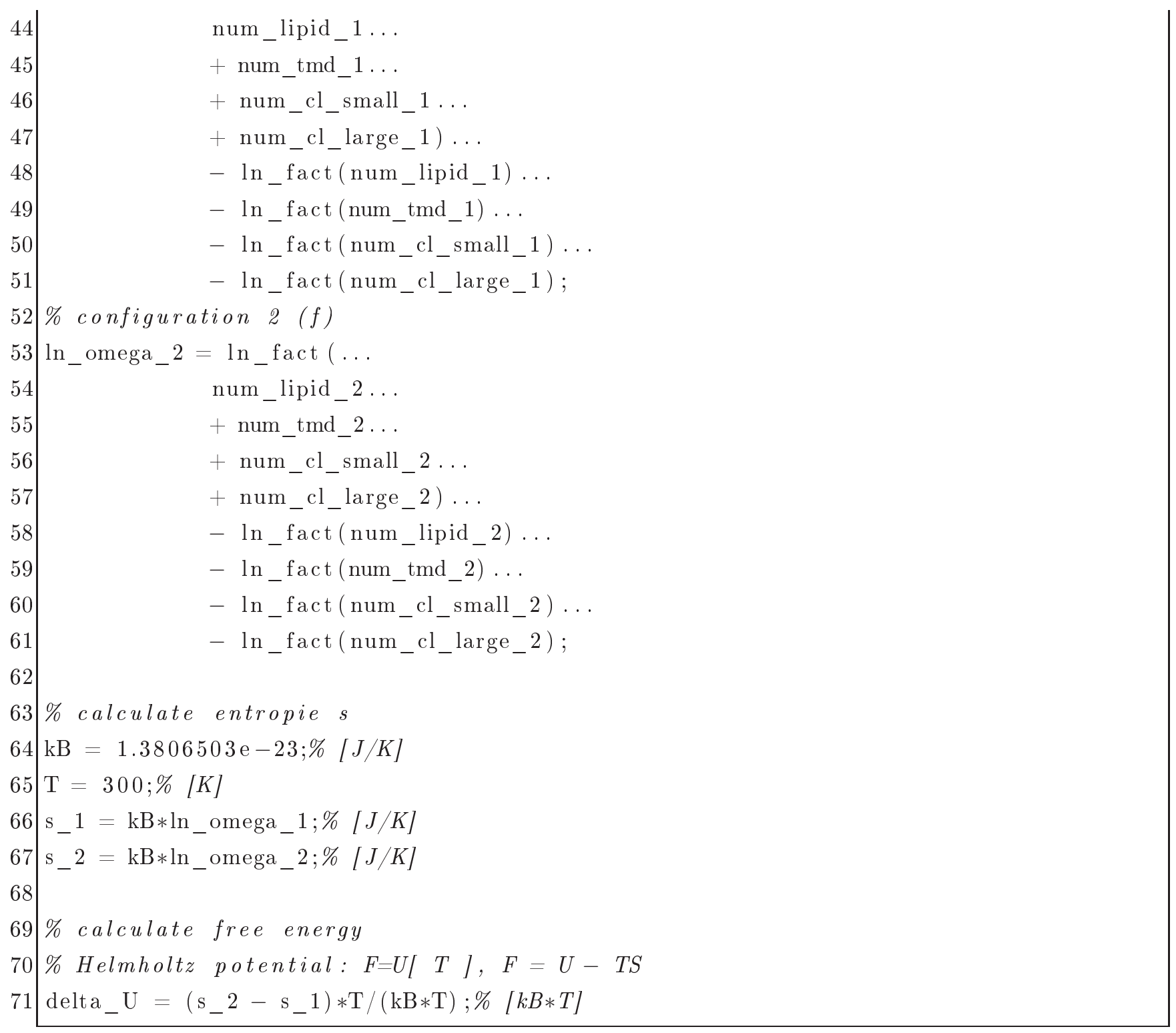

\section{A.3.2 ln_fact.m}

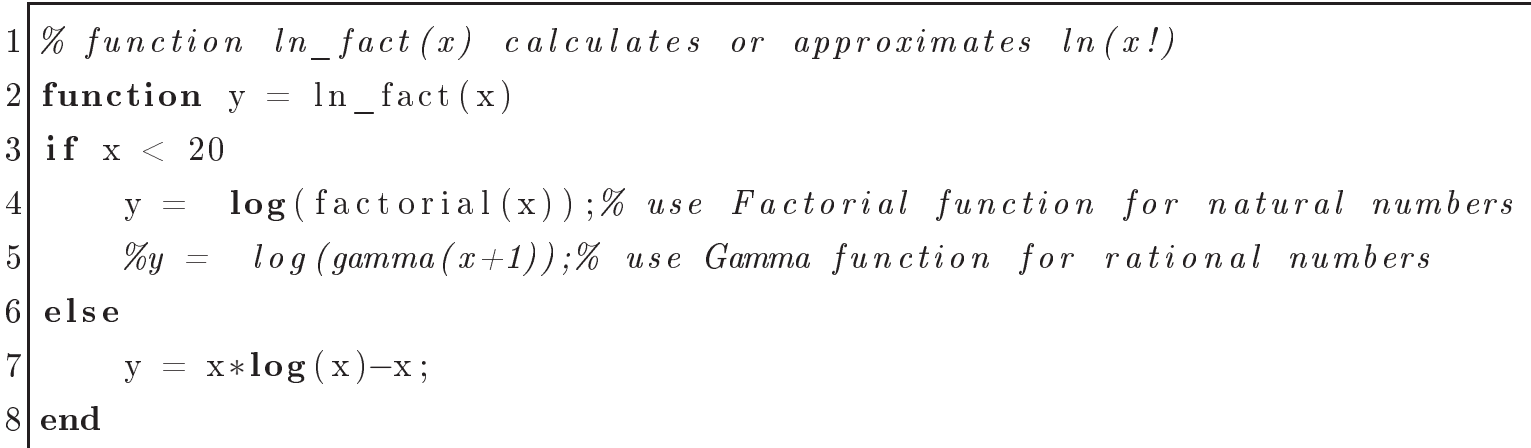




\section{Appendix B}

\section{List of Publications}

- S. Castorph, D. Riedel, L. Arleth, M. Sztucki, R. Jahn, M. Holt, and T. Salditt, Structure Parameters of Synaptic Vesicles Quantified by Small-Angle X-Ray Scattering, Biophysical Journal 98, 1200-1208 (2010).

- S. Castorph, L. Arleth, M. Sztucki, U. Vainio, S. K. Ghosh, M. Holt, R. Jahn, and T. Salditt, Synaptic Vesicles Studied by SAXS: Derivation and Validation of a Model Form Factor, Journal of Physics: Conference Series 247, 012015 (17pp) (2010).

- S. K. Ghosh, S. Castorph, O. Konovalov, R. Jahn, M. Holt, and T. Salditt, In vitro study of interaction of synaptic vesicles with lipid membranes, New Journal of Physics 12, 105004 (24pp) (2010).

- S. Castorph, S. Schwarz Henriques, M. Holt, D. Riedel, R. Jahn, and T. Salditt, Synaptic Vesicles Studied by Dynamic Light Scattering, in preparation.

- S. K. Ghosh, S. Castorph, O. Konovalov, R. Jahn, M. Holt, and T. Salditt, Effect of $\mathrm{Ca}^{2+}$ ions on lipid monolayer structure and interaction with synaptic vesicles, in preparation. 


\section{Appendix C}

\section{Acknowledgements}

It has been a great pleasure and honor for me to work together with many outstanding colleagues, and I am deeply indebted to everybody who has contributed to this work.

First and foremost, I thank Tim Salditt for proposing the interesting topic and for the outstanding and highly inspiring support of this work; I am particulary grateful for the numerous possibilities to present my work at national and international conferences and meetings providing ample opportunities to exchange exciting ideas with many outstanding scientists. I thank Reinhard Jahn for his genuine interest in this project, and for his continuous support. I thank Marcus Müller for useful discussions related to biophysical modeling of membrane systems and for co-refereeing this thesis.

I thank Helmut Grubmüller, Rainer Ulbrich, Sarah Köster and Claus Ropers for agreeing to be examination board members, and for their unreserved support regarding the organization of the disputation.

I thank Matthew Holt from the Jahn laboratory at the Department of Neurobiology at the MaxPlanck-Institute for Biophysical Chemistry in Göttingen, for sharing his profound biochemical knowledge and for his comprehensive support of the project, including the preparation of numerous synaptic vesicle and proteo-liposome samples, help during synchrotron experiments, many highly useful suggestions and discussions and the proof-reading of this thesis.

I thank Dietmar Riedel from the Electron Microscopy Group at the Department of Neurobiology at the Max-Planck-Institute for Biophysical Chemistry in Göttingen, for numerous electron microscopy measurements, including the quantitative analysis of particle radii of several thousand synaptic vesicle and liposome images.

I thank Lise Arleth for actively sharing her profound knowledge related to model building and form factor optimization, and her very obliging hospitality at the Institut for Grundvidenskab og Miljø Biofysik at the University of Copenhagen.

I thank Sarah Schwarz Henriques for her important fundamental work and help, related to the characterization of the size distribution of synaptic vesicles using light scattering and 
asymmetric-flow field-flow fractionation of synaptic vesicle samples, as laid down in her diploma thesis, and for support during synchrotron experiments at HASYLAB.

I thank Sajal Kumar Ghosh for his work and comprehensive support related to cell free interaction systems and fusion systems, including several mutual synchrotron experiments at ESRF and HASYLAB, and for carefully proof-reading this mansuscript.

I thank Michael Sztucki and Theyencheri Narayanan for excellent support of the synchrotron experiments at beamline ID-2 at the European Synchrotron Radiation Source in Grenoble.

I thank Ulla Vainio and Rainer Gehrke for excellent support of the synchrotron experiments at beamline B1 at the Hamburger Synchrotronstrahlungslabor at Deutsches Elektronen-Synchrotron. I thank Otto Glatter for enlightening discussions regarding inversion of scattering data, and his very obliging hospitality at his Institut für physikalische Chemie at the Karl-FranzensUniversität in Graz.

I thank Martin Hömberg from the Institut für Theoretische Physik at the Georg-AugustUniversität Göttingen, for highly useful discussions and help regarding form factor calculations. I thank Thorsten Hohage from the Institut für Numerische und Angewandte Mathematik at the Georg-August-Universität Göttingen, and Thomas Hotz from the Institut für Mathematische Stochastik at the Georg-August-Universität Göttingen for their help and support concerning regularization and inversion of scattering data.

I thank Hauke Schollmeyer and Gudrun Lotze for excellent support during the small-angle x-ray scattering experiments at ID-2, and the related raw data treatment and processing.

I thank Thorsten Gronemann and Jochen Herbst for outstanding support related to technical issues in the laboratories of the Institut für Röntgenphysik, in particular during preparation of synchrotron experiments.

I thank Gottfried Mieskes for outstanding support related to the use of technical equipment in the Jahn laboratory at the Department of Neurobiology at the Max-Planck-Institute for Biophysical Chemistry in Göttingen and Maria Druminski for help with the synaptic vesicle preparations. Further, I thank Matias Hernandez for sharing optimized parameter settings for the asymmetric-flow field-flow fractionation apparatus.

I thank the entire staff of the Institut für Röntgenphysik for their outstanding support and the highly inspiring working atmosphere that made this work possible.

I sincerely apologize to all colleagues whose contributions, although relevant for the success of the project, could not be included in this thesis, or are not explicitly mentioned here.

This work was financially supported by the "Excellence Cluster Initiative 171/ Deutsche Forschungsgemeinschaft (DFG) Research Center 103 - Center for Molecular Physiology of the Brain", DFG SFB 803 Collaborative Research Centre "Functionality Controlled by Organization in and between Membranes", DFG SFB 755 Collaborative Research Centre "Nanoscale Photonic Imaging" as well as by the Max Planck Society. The European Synchrotron Radiation Facility in 
Grenoble and the Hamburger Synchrotronstrahlungslabor at Deutsches Elektronen-Synchrotron are acknowledged for beamtime and excellent working conditions. 


\section{Vita}

Simon Johannes Castorph

geboren am 13. Juni 1980 in Göttingen,

Staatsangehörigkeit: deutsch

\section{Schulbildung}

1987 - $2000 \quad$ Rudolf Steiner-Schule Nürnberg

1998

Latinum, Pirckheimer-Gymnasium Nürnberg

2000

Allgemeine Hochschulreife, Sigmund-Schuckert-Gymnasium Nürnberg

\section{Studium}

$2000-2002$

2002

$2002-2003$

$2003-2005$

2005

seit 2006

Physik-Studium an der Friedrich-Alexander-Universität Erlangen-Nürnberg

Molekularbiologie-Studium an der University of California, Los Angeles

Physik-Studium am Imperial College of Science, Technology and Medicine,

London

Physik-Studium an der Friedrich-Alexander-Universität Erlangen-Nürnberg

Diplom in Physik, Titel der Diplomarbeit:

„Strukturuntersuchungen an biogenem Hoch-Magnesium Calcit“,

Lehrstuhl für Kristallographie und Strukturphysik,

Friedrich-Alexander-Universität Erlangen-Nürnberg

wissenschaftlicher Mitarbeiter am Institut für Röntgenphysik,

Georg-August-Universität Göttingen 